\title{
MANIFOLD PHENOMENA IN THE THEORY OF POLYHEDRA
}

\author{
BY \\ ETHAN AKIN
}

Introduction. When can an isotopy be covered by an ambient isotopy? Let us restrict attention to the compact p.l. category.

Hudson and Zeeman have shown that a locally unknotted isotopy of a manifold in a manifold can be covered by an ambient isotopy of the big manifold. By Zeeman's codimension $\geqq 3$ unknotting theorem, an isotopy of manifolds is locally unknotted if the codimension is greater than or equal to 3 . Hence, any isotopy of a manifold in a manifold of dimension at least 3 higher can be covered. Lickorish has generalized Zeeman's unknotting theorem to the case of a proper embedding of a cone in a ball of dimension at least three higher. From this, Hudson has shown any isotopy of a polyhedron in a manifold can be covered if the polyhedron has codimension at least 3 .

As a modest aim we would like a criterion of local unknottedness of a polyhedron in a manifold so that the original Hudson-Zeeman theorem would generalize.

What we actually obtain is more general. We present a characterization of those isotopies of a polyhedron in a polyhedron which can be covered by ambient isotopies. Perhaps surprisingly, this question admits a rather elegant general solution.

Intrinsic Dimension. Our major tool is the theory of intrinsic dimension developed by Armstrong. Given a polyhedral pair $\left(X, X_{0}\right)$ and a point $x \in X_{0}$, we define the intrinsic dimension of $x$ in $\left(X, X_{0}\right)$, denoted $d\left(x ; X, X_{0}\right)$, to be

$$
\max \left\{t: \text { link pair of } x \text { in }\left(X, X_{0}\right) \text { is a } t \text {-fold suspension }\right\},
$$

or equivalently,

$\max \left\{t:\right.$ there is a triangulation of $\left(X, X_{0}\right)$ with $x$ in the interior of a $t$-simplex $\}$.

Let

$$
I^{i}\left(X, X_{0}\right)=\left\{x \in X_{0}: d\left(x ; X, X_{0}\right) \leqq i\right\}
$$

called the intrinsic $i$-skeleton of $\left(X, X_{0}\right)$. For the absolute notion we associate the polyhedron $X$ with the pair $(X, X)$, i.e. $d(x ; X)=d(x ; X, X)$ and $I^{i}(X)=I^{i}(X, X)$.

The first fact about intrinsic dimension is that $I^{i}\left(X, X_{0}\right)$ is a subcomplex of any triangulation of $\left(X, X_{0}\right)$.

Received by the editors November 12, 1968 . 
The second fundamental fact is that $I^{i}\left(X, X_{0}\right)-I^{i-1}\left(X, X_{0}\right)$ is an $i$-manifold. In fact, the homogeneity of $I^{i}\left(X, X_{0}\right)-I^{i-1}\left(X, X_{0}\right)$ is an ambient fact, that is, two points of $X_{0}$ lie in the same component of $I^{i}\left(X, X_{0}\right)-I^{i-1}\left(X, X_{0}\right)$ for some $i$, iff there is an ambient isotopy of $\left(X, X_{0}\right)$ taking one point to the other.

The most useful form of these homogeneity facts is in the simplicial aspect. Let ( $\left.K, K_{0}\right)$ be a complex pair with $B=A C \in K_{0}$ and assume that $A, B \in I^{i}\left(K, K_{0}\right)$ $-I^{i-1}\left(K, K_{0}\right)$. Then there is an autohomeomorphism of $\left(K, K_{0}\right)$ supported by $\mathrm{St}^{\circ}(A ; K)$ and taking $B$ to $x \dot{A} C$ where $x \in \operatorname{Int} B$, i.e. we can ambient isotope the "nice face" $A$ of $B$ into the interior of $B$.

Using intrinsic dimension there is a definition of local unknottedness which reduces to the usual one in the case of manifold pairs. Call a polyhedral pair ( $\left.X, X_{0}\right)$ locally unknotted at $x \in X_{0}$ if $d\left(x ; X, X_{0}\right)=d\left(x ; X_{0}\right)$. Thus, if $\left(X, X_{0}\right)$ is a manifold pair and $\operatorname{dim} X_{0}=n$, then the set of points of $X_{0}$ at which the pair is locally knotted is precisely $=I^{n-1}\left(X, X_{0}\right)$. It is easily checked that since $S^{0}$ unknots in any sphere this actually $=I^{n-2}\left(X, X_{0}\right)$. Thus, the intrinsic skeleta of the pair decompose $X_{0}$ into submanifolds according to the number of times the link sphere pair desuspends as a pair.

Regular neighborhoods and collapsing. The application of intrinsic dimension from which the other applications flow is to the theory of regular neighborhoods in polyhedra, as developed by Cohen.

First, we define relative collapsing. We say that $X$ collapses to $X^{\prime}$ relative to $X_{0}$, denoted $\left(X \mid X_{0}\right) \searrow X^{\prime}$, if there is a collapse of $X$ to $X^{\prime}$, i.e. a sequence $X=X^{n}, \ldots$, $X^{0}=X^{\prime}$ with $B^{i}=\mathrm{Cl}\left(X^{i+1}-X^{i}\right)$ a p.l. ball with $B^{i} \cap X^{i}=F^{i}$ a face, for all $i$, which satisfies the additional condition $B^{i} \cap X_{0} \subset \mathrm{Cl}\left(\partial B^{i}-F^{i}\right)$.

The definition is chosen so that a relative collapse will preserve the homeomorphism type of the relative regular neighborhood, i.e. we want a regular neighborhood of $X \bmod X_{0}$ to be a regular neighborhood of $X^{\prime} \bmod X_{0}$ in an ambient manifold, and without the additional condition things can go wrong.

Thus, if we let $X$ be a simplex $[a b c]$ and $X_{0}=[b x]$ where $x \in$ Int [ac] then in a manifold, a regular neighborhood of $X \bmod X_{0}$ is two balls joined along the simplex $[b x]$. We can collapse $X$ to $X^{\prime}=[a b x] \cup[x c]$ (not relative to $X_{0}$ ) and a regular neighborhood of $X^{\prime} \bmod X_{0}$ is two balls joined along a point.

That relative collapsing does preserve homeomorphism type (and more) of regular neighborhoods in manifolds follows from the more general results in polyhedra.

Now assume that $X<Q_{0}<Q$. We call a collapse $\left(X \mid X_{0}\right) \searrow X^{\prime}$ homogenous in $\left(Q, Q_{0}\right)$ if $d\left(x ; Q, Q_{0}\right)$ is constant for $x$ varying in each $X^{i+1}-X^{i}$ and weakly homogenous if it is constant for $x$ varying in each $X^{i+1}-\left(X^{i} \cup X_{0}\right)$. Note that it does not follow that $d\left(x ; Q, Q_{0}\right)$ is constant as $x$ varies all over $X-X^{\prime}$. If there exists such a homogenous (resp. weakly homogenous) collapse, we will write $\left(X \mid X_{0}\right)_{h} \searrow X^{\prime}$ in $\left(Q, Q_{0}\right)$ (resp. $\left(X \mid X_{0}\right)_{w \downarrow} X^{\prime}$ in $\left.\left(Q, Q_{0}\right)\right)$.

The phrase homogenous collapse was introduced by Stallings in the absolute 
case $\left(X_{0}=\varnothing\right.$ and $Q=Q_{0}$ ), and in that case his definition is equivalent to ours though he does not use the notion of intrinsic dimension.

We note that if $\left(X \mid X_{0}\right)_{n} \searrow X^{\prime}$ (or $\left.w_{w}\right)$ in $Q_{0}$ and $\left(Q, Q_{0}\right)$ is locally unknotted, then $\left(X \mid X_{0}\right)_{h} \searrow X^{\prime}$ (resp. $\left.w \downarrow\right)$ in $\left(Q, Q_{0}\right)$.

THEOREM. Let $X_{0}<X<Q_{0}<Q$ be polyhedra with $\left(X \mid X_{0}\right)_{n \searrow} X^{\prime}$ in $\left(Q, Q_{0}\right)$. If $\left(V, V_{0}\right)$ is a regular neighborhood of $X \bmod X_{0}$ in $\left(Q, Q_{0}\right)$ then $\left(V, V_{0}\right)$ is a regular neighborhood of $X^{\prime} \bmod X^{\prime} \cap X_{0}$ in $\left(Q, Q_{0}\right)$.

THEOREM. Let $X_{0}<X<Q_{0}<Q$ be polyhedra with $\left(X \mid X_{0}\right)_{w} \backslash X^{\prime}$ in $\left(Q, Q_{0}\right)$. If $\left(V, V_{0}\right)$ and $\left(U, U_{0}\right)$ are regular neighborhoods of $X \bmod X_{0}$ and $X^{\prime} \bmod X_{0}$ respectively, in $\left(Q, Q_{0}\right)$ then there is a homeomorphism $f:\left(V, V_{0}\right) \rightarrow\left(U, U_{0}\right)$ which preserves the boundaries and is the identity on $X^{\prime}$.

The idea of the proof is quite simple. We triangulate and use induction to reduce the problem to one simplicial collapse, i.e. $X=X^{\prime}+B$ where $B=b A$ and $B \cap X^{\prime}$ $=b A$. The condition that the collapse is relative says that $B \cap X_{0}<A$. There are two cases, i.e. $B \cap X_{0}=A$ or $B \cap X_{0}<\partial A$. Then in the second theorem $A$ is a "nice face" of $B$ if $B \cap X_{0}<\partial A$ and in the first theorem $A$ is a "nice face" of $B$ regardless.

Using the simplicial method of "parametrization", taken from Cohen, we construct directly regular neighborhoods of both $X^{\prime} \bmod X_{0}$ and $X^{\prime} \cup x b A \bmod X_{0}$ in the second case and both $X^{\prime} \bmod X_{0}$ and $X^{\prime} \cup x b \dot{A}$ moci $X_{0} \cup x \dot{A}$ in the first case.

Then using the fact that $A$ is a "nice face" of $B$ we find an ambient homeomorphism of $\left(Q, Q_{0}\right)$ which is the identity on $X^{\prime}$ and maps $X^{\prime} \cup x b A$ to $X^{\prime} \cup B=X$. This will push the regular neighborhoods out to obtain regular neighborhoods of both $X^{\prime} \bmod X_{0}$ and $X \bmod X_{0}$ in each case.

The remaining possibility is that we are in the first case of the second theorem and $A$ is not a "nice face" of $B$. In this case the homeomorphism $f$ is just constructed directly using the method of parametrization.

From the above theorems we can then derive several characterizations of a regular neighborhood of $X$ mod $X_{0}$ in $Q$ using collapsing.

Cone unknotting. Given an embedding $f: a X \rightarrow b Y$ with $f^{-1}(Y)=X$ (i.e. a proper embedding), call $f$ unknotted if there exists a homeomorphism $\phi: b Y \rightarrow b Y$ rel $Y$, such that $\phi f$ is the cone on $f \mid X: X \rightarrow Y$. This definition, due to Lickorish, is much stronger than merely assuming that $(b Y, f(a X)) \cong c(Y, f(X))$ rel $Y$. In fact, in most applications, $f$ is a self-homeomorphism of a subcone of $b Y$, which we are attempting to cover by a self-homeomorphism of $b Y$. Such a covering homeomorphism will exist iff, regarded as an embedding, the little homeomorphism is unknotted.

The confusion between the two kinds of unknotting arises because for ball pairs they are equivalent. This is because an unknotted ball pair is of the form $(\dot{\Delta} * \bar{\Delta}, \bar{\Delta})$ and if $f: \bar{\Delta} \rightarrow \bar{\Delta}$ is a self-homeomorphism then $\dot{\Delta} * f$ is a covering self-homeomorphism of $\dot{\Delta} * \bar{\Delta}$. 
THEOREM. Let $f: a X \rightarrow b Y$ be a proper embedding. Then $f$ is unknotted iff

1. For every $x \in X, d(f(t x+(1-t) a) ; b Y, f(a X))$ is constant for $0<t<1$ (conewise homogeneity), and

2. $(b Y \mid Y)_{w \searrow} f(a X)$ in $b Y$.

The second condition is just to insure that $b Y$ is homeomorphic to a regular neighborhood of $f(a X)$ mod $f(X)$ in $b Y$. Then the result follows from:

THEOREM. Let $f: a X \rightarrow Q$ be an embedding, which is conewise homogenous. Then if $V$ is a regular neighborhood of $f(a X) \bmod f(X)$ in $Q$, then there exists a homeomorphism $\phi: V \rightarrow b \dot{V}$ (where $\dot{V}=\mathrm{Bdry} V \cup f(X)$ ) such $\phi \mid \dot{V}$ is the identity and $\phi f$ is the cone on $f \mid X: X \rightarrow \dot{V}$.

To get an idea of the proof note that the cone structure gives an obvious "conewise" collapse $(f(a X) \mid f(X)) \searrow f(a)$. Conewise homogeneity says precisely that this collapse is weakly homogenous in $(Q, f(a X))$. It follows that $(V, f(a X))$ is homeomorphic to a regular neighborhood of $f(a)$ in $(Q, f(a X))$. Thus, the weak "set-unknottedness" condition follows from the theorems of the regular neighborhood section.

The sharper "map-unknottedness" condition of the theorem is proved by going back to the appropriate regular neighborhood theorem and repeating the proof, taking care to preserve the additional structure on the polyhedron $f(a X)$ introduced by the cone structure of $a X$.

Note that the $d(f(t x+(1-t) a) ; f(a X))$ is constant for $0<t<1$. Thus, if $(Q, f(a X))$, is locally unknotted, conewise homogeneity always holds. Thus, for the first time, the oft-returning "red herring" effect of local unknottedness appears:

In general, the purpose of assuming local unknottedness of a pair $\left(X, X_{0}\right)$ is to transform conditions on the intrinsic dimension in $\left(X, X_{0}\right)$ to conditions on the intrinsic dimension in $X_{0}$, which are usually trivial.

On the other hand, if $(b Y, f(a X)) \cong b^{*}(Y, f(X))$ rel $Y$, then the collapsing condition $(b Y \mid Y) w \searrow f(a X)$ in $b Y$ holds.

Thus, if $a(Y, X)$ is a locally unknotted cone pair then any self-homeomorphism of $a X$ rel $X$ can be covered by a self-homeomorphism of $a Y$ rel $Y$.

Codimension $\geqq 3$. Using sunny collapsing at a crucial stage, we can strengthen the cone unknotting theorem when codimenion is $\geqq 3$.

THEOREM. Let $f: a X \rightarrow b Y$ be a proper embedding with $\operatorname{dim} f^{-1} I^{i}(Y) \leqq i-3$ for all $i$. If $f(a)=b$, and $f^{-1}\left(b I^{i}(Y)\right)=a f^{-1} I^{i}(Y)$ then $f$ is unknotted.

From this theorem we obtain Lickorish's theorem and also a criterion for local unknottedness in codimension $\geqq 3$.

Covering isotopies. From the cone unknotting theorems we can get our covering isotopy theorems using the Hudson-Zeeman methods.

We define an isotopy $F: X \times I \rightarrow Y \times I$ to be locally collared if for every subinterval $J$ of $I,(Y \times \dot{J}, F(X \times \dot{J})) \subset(Y \times J, F(X \times J))$ is locally collared. Rourke has 
proven that an isotopy is locally collared iff there is an ambient isotopy of $Y$ covering the track of $F$, i.e. there exists $G: Y \times I \rightarrow Y \times I$ such that $G_{t} F_{0}(X)=F_{t}(X)$.

THEOREM. Let $F: X \times I \rightarrow Y \times I$ be an isotopy. F can be covered by an ambient isotopy of $Y$ iff

1. $F$ is locally collared, and

2. for each $x \in X, d(F(x, t) ; Y \times I, F(X \times I))$ is constant for $0<t<1$.

Call an isotopy $F: X \times I \rightarrow Y \times I$ locally unknotted if it is locally collared and $(Y \times I, F(X \times I))$ is locally unknotted. Since $d(F(x, t) ; F(X \times I))$ is constant $0<t<1$, we have another "red herring" phenomenon:

COROLlary. Any locally unknotted isotopy can be covered by an ambient isotopy.

Now if $\left(X, X_{0}\right)$ is a polyhedral pair and $F: X_{0} \times I \rightarrow X_{0} \times I$ is an ambient isotopy of $X_{0}$, then regarded as an isotopy in $X$ it is clearly locally collared and hence we have

Corollary. If $F: X_{0} \times I \rightarrow X_{0} \times I$ is an ambient isotopy then $F$ can be covered by an ambient isotopy of $X$ iff $F^{-1}\left(I^{i}\left(X, X_{0}\right) \times I\right)=I^{i}\left(X, X_{0}\right) \times I$.

From this we get a relative covering isotopy theorem.

TheOREM. Let $\left(X, X_{0}\right)$ be a polyhedral pair and $Y<X$. Assume $F: X_{0} \times I \rightarrow X_{0} \times I$ is an ambient isotopy of $X_{0}$ rel $X_{0} \cap Y$. If $F$ can be covered by an ambient isotopy of $X$, then it can be covered by an ambient isotopy of $X$ rel $Y$.

For we can extend the obvious ambient isotopy of $X_{0} \cup Y$.

For local unknottedness we get the following:

COROLlaRY. Let $\left(X, X_{0}\right)$ be a locally unknotted pair, then any ambient isotopy of $X_{0}$ can be covered by an ambient isotopy of $X$.

Finally, from the codimension $\geqq 3$ cone unknotting theorem, we obtain a corresponding isotopy theorem:

THEOREM. Let $F: X \times I \rightarrow Y \times I$ be an isotopy with $\operatorname{dim} F_{0}^{-1} I^{i}(Y) \leqq i-3$. Then $F$ can be covered by an ambient isotopy of $Y$ iff $F^{-1}\left(I^{i}(Y) \times I\right)=F_{0}^{-1}\left(I^{i}(Y)\right) \times I$.

As a corollary of this we have Hudson's result that any isotopy of a polyhedron of codimension $\geqq 3$ in a manifold can be covered by an ambient isotopy.

Extending triangulations. Armstrong's theorem that any triangulation of a locally unknotted submanifold extends to a triangulation of the manifold generalizes to the following:

THEOREM. Let $\left(X, X_{0}\right)$ be a polyhedral pair and $\left(K_{0} ; h\right)$ a triangulation of $X_{0}$. Then $\left(K_{0} ; h\right)$ extends to a triangulation of $X$ iff $h^{-1} I^{i}\left(X, X_{0}\right)$ is a subcomplex of $K_{0}$ for all $i$. 
Since $I^{i}\left(X_{0}\right)=I^{i}\left(X, X_{0}\right)$ iff the pair is locally unknotted, we have another "red herring" effect.

Corollary. Let $\left(X, X_{0}\right)$ be a locally unknotted polyhedral pair. Then any triangulation of $X_{0}$ extends to a triangulation of $X$.

General position. We call a homotopy $F: X \times I \rightarrow Y$ homogenous if for every $x \in X d(F(x, t) ; Y)$ is constant for $t \in I$.

Using the isotopy theorems we can prove results on homogenous homotopy of maps:

THEOREM. Let $f: X \rightarrow Y$ be a map such that $f \mid X_{0}$ is nondegenerate (where $X_{0}<X$ ) and $\operatorname{dim} f^{-1} I^{i}(Y) \leqq i$. Then there exists a homotopy of $f$, homogenous and $\operatorname{rel} X_{0}$, to a nondegenerate map.

THEOREM. Let $f: X \rightarrow Y$ be a nondegenerate map, then $f$ can be homogeneously homotoped to a map $g: X \rightarrow Y$ such that $\operatorname{dim} S(f)-f^{-1} I^{i-1}(Y) \leqq 2 \operatorname{dim} X-i$.

We also mention some conjectures which relate these results to those of Stallings about $N D(n)$-spaces.

Acknowledgement. I would like to thank M. A. Armstrong, C. P. Rourke and Ralph Reid for many helpful chats. To George Cooke, I owe thanks not only for mathematical help but also for the unfailing confidence and warm friendship which helped to incubate this work. Finally, I would like to thank my advisor Marshall Cohen for his vast mathematical aid, without which this paper could probably not have been written by me, and for his dauntless editorial work, without which it could probably not have been read by anyone else.

I. Notation and background information. We will follow the standard treatments of p.l. topology: Zeeman [Z], Hudson $[\mathbf{H}]$ and Stallings [S]. These do not completely overlap so we will refer mostly to $[\mathbf{H}]$ and occasionally to $[\mathbf{S}]$.

Our polyhedra, polyhedral pairs, polyhedra with (finite) families of subpolyhedra, denoted $X,\left(X, X_{0}\right),\left(X,\left\{X_{i}\right\}\right)$ will be assumed to have a given family of compatible locally finite triangulations, denoted $(K ; h),\left(K, K_{0} ; h\right),\left(K,\left\{K_{i}\right\}, h\right),\left(K,\left\{K_{i}\right\} ; h\right)$ for complexes, complex pairs and complexes with a family of subcomplexes. All maps are assumed to be p.l.

For $A \in K$, we will denote by $\mathrm{Lk}(A ; K)$, St $(A ; K)$ and $\mathrm{St}^{\circ}(A ; K)$ the link, star and open star of $A$ in $K$. Note that these are finite, by local finiteness of $K$. For pairs, we will define $\mathrm{Lk}\left(A ; K, K_{0}\right)$, for example, to equal $\left(\mathrm{Lk}(A ; K), \operatorname{Lk}\left(A ; K_{0}\right)\right)$ for $A \in K_{0}$.

Following Armstrong [ $\left.\mathbf{A}_{1}\right]$, for $x \in X$, we will denote by $\operatorname{Lk}(x ; X)$ any $\mathbf{L k}$ $\left(h^{-1}(x) ; K\right)$ where $(K ; h)$ is a triangulation of $X$ and $h^{-1}(x)$ is a vertex of $K$. Similarly, for $x \in X_{0}$ define $\operatorname{Lk}\left(x ; X, X_{0}\right)$ using any triangulation of the pair. These are well defined up to homeomorphism, or homeomorphism of pairs, respectively. Similarly, define St $(x ; X)$ and St $\left(x ; X, X_{0}\right)$. 
We recall that joining gives a well-defined functor of pairs of compact polyhedra to compact polyhedra. Denoted $X * Y$. Points of $X * Y$ are denoted $t x+(1-t) y$ with $x \in X, y \in Y$ and $t \in I$. If $f: X \rightarrow X_{0}$ and $g: Y \rightarrow Y_{0}$ then $f * g: X * Y \rightarrow$ $X_{0} * Y_{0}$ maps by $f * g(t x+(1-t) y)=t f(x)+(1-t) g(y)$. If $X$ is one point, $c$, then $c * Y$ or $c Y$ is called the cone on $Y$ and if $X$ is two points, i.e. a 0 -sphere $S^{0}$, then $S^{0} * Y$ or $\Sigma Y$ is called the suspension of $Y$. The iterated suspension $\Sigma \Sigma^{n} Y=\Sigma^{n+1} Y$ $=S^{n} * Y$. We will often use the calculation of the link of a point of the join:

$$
\begin{aligned}
\operatorname{Lk}(t x+(1-t) y ; X * Y) & =X * \operatorname{Lk}(y ; Y), & & t=0 \\
& =\Sigma \operatorname{Lk}(x ; X) * \operatorname{Lk}(y ; Y), & & 0<t<1 \\
& =\operatorname{Lk}(x ; X) * Y, & & t=1 .
\end{aligned}
$$

This has an obvious generalization to $X *\left(Y, Y_{0}\right)$.

Note that any join factor and hence any cone or suspension factor is assumed to be compact.

The product of arbitrary polyhedra, denoted $X \times Y$, is defined and the identity for the link is

$$
\text { St }((x, y) ; X \times Y)=\operatorname{St}(x ; X) \times \operatorname{St}(y ; Y) .
$$

This implies that

$$
\operatorname{Lk}((x, y) ; X \times Y)=\operatorname{Lk}(x ; X) * \operatorname{Lk}(y ; Y)
$$

by the Alexander trick which we now describe. Given finite complexes $K$ and $L$, we can triangulate $c K \times c L$ so that $\mathrm{St}((c, c) ; c K \times c L)=c K \times c L$ and

$$
\operatorname{Lk}((c, c) ; c K \times c L)=c K \times L \cup K \times c L
$$

(where the union is along $K \times L$ ). Furthermore, $c K \times L \cup K \times c L \cong K * L$ (see [S; $\$ 4.3])$.

Of particular interest is the case where $L$ is one point so $c L \cong I$. This gives:

$$
c K \times I=(c, 1) *((c K \times 0) \cup(K \times I))
$$

with $c K \times 1$ included as the subcomplex $(c, 1) *(K \times 1)$. This construction is also natural on subcomplexes of $K$.

The Alexander trick proves that a homeomorphism of a cone rel its base is isotopic to the identity rel its base. We define the isotopy (after triangulating) to be the map of $c K \times I$ to itself obtained by coning the given homeomorphism on $c K \times 0$ and the identity of $K \times I$.

As an indispensible tool we will require the general theory of relative regular neighborhoods as developed by Cohen in $[\mathbf{C}]$. Following him, we define $\left(V, V_{0}\right)$ to be a regular neighborhood of $X \bmod X_{0}$ in $\left(Y, Y_{0}\right)$, if there is a triangulation $\left(J, J_{0} ; h\right)$ of $\left(Y, Y_{0}\right)$ such that $K=h^{-1}(X), K_{0}=h^{-1}\left(X_{0}\right)$ and $J_{0}$ are full subcomplexes of $J$, and

$$
\left(V, V_{0}\right)=h N\left(K-K_{0} ; \eta J, \eta J_{0}\right)
$$


where $\eta$ is a derived subdivision of $J$. Define the boundary of the regular neighbor$\operatorname{hood}\left(\dot{V}, \dot{V}_{0}\right)$ by

$$
\left(\dot{V}, \dot{V}_{0}\right)=h \dot{N}\left(K-K_{0} ; \eta J, \eta J_{0}\right) \text {. }
$$

By [C; Lemma 2.13], $\dot{V}=\operatorname{Bdry}_{Y} V \cup\left(X_{0}\right)_{R}$ where $\left(X_{0}\right)_{R}=X_{0} \cap(X)_{R}$ and $(X)_{R}=\mathrm{Cl}_{Y}\left(X-X_{0}\right)$.

We will use much of the notation of $[\mathrm{C}]$, such as $D(A ; K)$ for dual cell, $\dot{D}(A ; K)$ for boundary of the dual cell, and so on. We will also depend heavily on the results of [C] particularly the parametrization lemma [C; Lemma 2.14], the uniqueness theorem [C; Theorems 3.1-3.4] and the stellar neighborhood theorem [C; Theorem 6.1].

We will require the theory of collapsing and mention, in particular, the principle of excision.

Let $X, X_{0}$ and $Y$ be polyhedra with $Y \cap X \subset X_{0}$. Then $X \searrow X_{0}$ iff $X \cup Y$ $\searrow X_{0} \cup Y$.

Finally, a little convention, for convenience in some formulae. We distinguish between $\varnothing$, the empty complex, and $\{0\}$ the complex consisting of the empty simplex 0. Define $\varnothing * X=\varnothing$ and $\{0\} * X=X$, if $X \neq \varnothing . \varnothing \times X=\varnothing$ and $\{0\} \times X=\{0\}$, if $X \neq \varnothing$. Also if $A \notin K$, then $\operatorname{define} \operatorname{Lk}(A ; K)=\varnothing$. We will always explicitly mention this convention whenever it is around.

II. Intrinsic dimension. We give three equivalent definitions of intrinsic dimension:

Definition 1. Let $\left(X, X_{0}\right)$ be a polyhedral pair and $x \in X_{0}$. Then we define the intrinsic dimension of $x$ in $\left(X, X_{0}\right)$, denoted $d\left(x ; X, X_{0}\right)$ to be

a. the greatest $t$, such that $\operatorname{Lk}\left(x ; X, X_{0}\right)$ is a $t$-fold suspension,

b. the greatest $t$, such that there exists an embedding $f: \Delta^{t} \times c\left(Y, Y_{0}\right) \rightarrow\left(X, X_{0}\right)$ such that $f^{-1}\left(X_{0}\right)=\Delta^{t} \times c Y_{0}$ and $f\left(\Delta^{t} \times c Y\right.$ ) is a neighborhood of $x=f(d, c)$ (for $d$ some element of Int $\Delta^{t}$ ),

c. the greatest $t$, such that there exists a triangulation $\left(K, K_{0} ; h\right)$ of $\left(X, X_{0}\right)$ with $h^{-1}(x)$ in the interior of a $t$-simplex of $K_{0}$.

\section{Proof of the equivalence.}

$\mathrm{a} \rightarrow$ b. Lk $\left(x ; X, X_{0}\right) \cong \Sigma^{t}\left(Y, Y_{0}\right) \rightarrow$ St $\left(x ; X, X_{0}\right) \cong \Delta^{t} *\left(Y, Y_{0}\right)$ with $x$ corresponding to an interior point of $\Delta^{t}$. But a regular neighborhood of $\Delta$ in $\Delta *\left(Y, Y_{0}\right)$ is homeomorphic to $\Delta^{t} \times c\left(Y, Y_{0}\right)$.

$\mathrm{b} \rightarrow \mathrm{a} . \Sigma^{t}\left(Y, Y_{0}\right) \cong \mathrm{Lk}\left((d, c) ; \Delta^{t} \times c\left(Y, Y_{0}\right)\right) \cong \mathrm{Lk}\left(x ; X, X_{0}\right)$ since $f(d, c)=x$.

$\mathrm{c} \rightarrow \mathrm{a}$. If $\left(K, K_{0} ; h\right)$ is a triangulation of $\left(X, X_{0}\right)$ with $h^{-1}(x) \in \operatorname{Int} A$, with $A \in K_{0}$ and $\operatorname{dim} A=t$, then $\operatorname{Lk}\left(x ; X, X_{0}\right) \cong \dot{A} * \operatorname{Lk}\left(A ; K, K_{0}\right) \cong \Sigma^{t} \operatorname{Lk}\left(A ; K, K_{0}\right)$.

a $\rightarrow$ c. Let $\left(K, K_{0} ; h\right)$ be a triangulation of $\left(X, X_{0}\right)$ with $h^{-1}(x)$ a vertex of $K_{0}$. As in the proof $\mathrm{a} \rightarrow \mathrm{b}$ we cone to get a homeomorphism $q$,

$$
q: \operatorname{St}\left(h^{-1}(x) ; K, K_{0}\right) \cong \Delta^{t} *\left(Y, Y_{0}\right)
$$

with $q\left(h^{-1}(x)\right)=d_{0}$ an interior point of $\Delta^{t}$. Let $d_{1} \in$ Int $\Delta^{t}$ such that $q^{-1}\left(d_{1}\right)$ lies in the interior of a simplex of $K_{0}$ of dimension at least $t$. 
Let $g: \Delta^{t} \cong \Delta^{t}$ rel $\partial \Delta^{t}$ with $g\left(d_{0}\right)=d_{1}$. Join with the identity on $Y$ and conjugate with $q$ to obtain

$$
k: \mathrm{St}\left(h^{-1}(x) ; K, K_{0}\right) \rightarrow \mathrm{St}\left(h^{-1}(x) ; K, K_{0}\right)
$$

satisfying $k \mid \mathrm{Lk}\left(h^{-1}(x) ; K\right)=$ identity and $k\left(h^{-1}(x)\right)=q^{-1}\left(d_{1}\right)$. Extend $k$ by the identity to the rest of $K$. Let $\left(K, K_{0} ; h k^{-1}\right)$ triangulate $\left(X, X_{0}\right) \cdot\left(h k^{-1}\right)^{-1}(x)=q^{-1}\left(d_{1}\right)$ and so lies in the interior of a simplex of $K_{0}$ of dimension at least $t$.

Note. The proof that $\mathrm{a} \rightarrow \mathrm{b}$ gave a homeomorphism

$$
q: \Delta^{t} *\left(Y, Y_{0}\right) \rightarrow \operatorname{St}\left(x ; X, X_{0}\right)
$$

taking $\partial \Delta^{t} *\left(Y, Y_{0}\right)$ to $\mathrm{Lk}\left(x ; X, X_{0}\right)$ and hence $q\left(\Delta^{t} * Y-\partial \Delta^{t} * Y\right)$ was open in $X$. Also, the resultant embedding $f: \Delta^{t} \times c\left(Y, Y_{0}\right) \rightarrow\left(X, X_{0}\right)$ satisfied the condition that $f\left(\left(\right.\right.$ Int $\left.\left.\Delta^{t}\right) \times(c Y-Y)\right)$ is open in $X$.

$d\left(x ; X, X_{0}\right)$ is sometimes called the ambient intrinsic dimension to distinguish it from the absolute intrinsic dimension in a polyhedron, rather than a polyhedral pair. We obtain the absolute definitions by identifying a polyhedron $X$, with the pair $(X, X)$. Thus conditions on link pairs, triangulation of pairs etc. become conditions on links and triangulations of polyhedra.

Definition 2. For $x \in X$, we define the intrinsic dimension of $x$ in $X$, denoted $d(x ; X)$, by setting $d(x ; X)=d(x ; X, X)$.

REMARKS. The definitions are obviously p.l. invariants, i.e. if $f:\left(X, X_{0}\right) \rightarrow$ $\left(Y, Y_{0}\right)$ is a homeomorphism, then $d\left(x ; X, X_{0}\right)=d\left(f(x) ; Y, Y_{0}\right)$.

An easy comparison of definitions shows that

$$
d\left(x ; X, X_{0}\right) \leqq \min \left(d(x ; X), d\left(x ; X_{0}\right)\right) .
$$

Definition 3. For a polyhedral pair $\left(X, X_{0}\right)$ or a polyhedron $X$, we define the intrinsic $i$-skeleton of $\left(X, X_{0}\right)($ resp. $X)$, denoted $I^{i}\left(X, X_{0}\right)$ (resp. $\left.I^{i}(X)\right)$ by:

$$
\begin{aligned}
I^{i}\left(X, X_{0}\right) & =\left\{x \in X_{0}: d\left(x ; X, X_{0}\right) \leqq i\right\} \\
I^{i}(X) & =I^{i}(X, X)=\{x \in X: d(x ; X) \leqq i\} .
\end{aligned}
$$

The name intrinsic $i$-skeleton is suggested by Zeeman's alternate definition of the intrinsic $i$-skeleton as the intersection of all possible $i$-skeleta. More precisely, part c. of Definition 1 implies

$$
I^{i}\left(X, X_{0}\right)=\bigcap\left\{h\left(K_{0}^{i}\right):\left(K, K_{0} ; h\right) \text { a triangulation of }\left(X, X_{0}\right)\right\} .
$$

That is, the intersection is taken over all triangulations of the polyhedral pair $\left(X, X_{0}\right)$. This implies that $I^{i}\left(X, X_{0}\right)$ is a closed subset of $X_{0}$.

THEOREM 4. Let $\left(K, K_{0} ; h\right)$ be a triangulation of a polyhedral pair $\left(X, X_{0}\right)$, then for all $i, h^{-1}\left(I^{i}\left(X, X_{0}\right)\right)$ is a subcomplex of $K_{0}$.

Proof. If $h^{-1}\left(x_{0}\right), h^{-1}\left(x_{1}\right) \in$ Int $A$ for $A$ is a simplex of $K_{0}$, then $\operatorname{Lk}\left(x_{i} ; X, X_{0}\right)$ $\cong \partial A * \operatorname{Lk}\left(A ; K, K_{0}\right), i=0,1$ and hence $d\left(x_{0} ; X, X_{0}\right)=d\left(x_{1} ; X, X_{0}\right)$. So intrinsic dimension is constant on the interior of any simplex of $K_{0}$. 
Hence, $h^{-1}\left(I^{i}\left(X, X_{0}\right)\right)$ is a union of open simplices of $K_{0}$ and as it is closed, it is a subcomplex.

Now we examine the intrinsic skeleta locally.

Lemma 5. Let $f: \Delta^{i} *\left(Y, Y_{0}\right) \rightarrow\left(X, X_{0}\right)$ be an embedding with $f^{-1}\left(X_{0}\right)=\Delta^{i} * Y_{0}$ and $f\left(\Delta^{i} * Y-\partial \Delta^{i} * Y\right)$ open in $X$, then for $d_{0}, d_{1} \in \operatorname{Int} \Delta^{t}, 0<t_{0}, t_{1}<1$ and $y \in Y_{0}$, we have

a. $d\left(f\left(d_{0}\right) ; X, X_{0}\right)=d\left(f\left(d_{1}\right) ; X, X_{0}\right) \geqq i$.

b. $d\left(f\left(t_{0} d_{0}+\left(1-t_{0}\right) y\right) ; X, X_{0}\right)=d\left(f\left(t_{1} d_{1}+\left(1-t_{1}\right) y\right) ; X, X_{0}\right)>i$.

Proof. a. $\operatorname{Lk}\left(f\left(d_{p}\right) ; X, X_{0}\right) \cong \operatorname{Lk}\left(d_{p} ; \Delta^{i} *\left(Y, Y_{0}\right)\right) \cong \partial \Delta^{i} *\left(Y, Y_{0}\right), p=0,1$.

b. $\quad \operatorname{Lk}\left(f\left(t_{p} d_{p}+\left(1-t_{p}\right) y\right) ; X, X_{0}\right) \cong \operatorname{Lk}\left(t_{p} d_{p}+\left(1-t_{p}\right) y ; \Delta^{i} *\left(Y, Y_{0}\right)\right)$

$$
\cong \Sigma \partial \Delta^{i} * \operatorname{Lk}\left(y ; Y, Y_{0}\right), p=0,1
$$

THEOREM 6. $I^{i}\left(X, X_{0}\right)-I^{i-1}\left(X, X_{0}\right)$ is an i-manifold.

Proof. If $d\left(x ; X, X_{0}\right)=i$, then there exists an embedding $f: \Delta^{i} *\left(Y, Y_{0}\right) \rightarrow$ $\left(X, X_{0}\right)$ such that $f^{-1}\left(X_{0}\right)=\Delta^{i} * Y_{0}, f\left(\Delta^{i} * Y-\partial \Delta^{i} * Y\right)$ is open in $X$ and $f^{-1}(x) \in$ Int $\Delta^{i}$. Lemma 5 implies that

$$
f\left(\Delta^{i} * Y_{0}-\partial \Delta^{i} * Y_{0}\right) \cap I^{i}\left(X, X_{0}\right)=f\left(\operatorname{Int} \Delta^{i}\right)
$$

and

$$
f\left(\text { Int } \Delta^{i}\right) \cap I^{i-1}\left(X, X_{0}\right)=\varnothing .
$$

Hence, $f\left(\right.$ Int $\left.\Delta^{i}\right)$ is a neighborhood of $x$ in $I^{i}\left(X, X_{0}\right)-I^{i-1}\left(X, X_{0}\right)$.

We now demonstrate the relationship between homogeneity of the open intrinsic $i$-skeleton as an $i$-manifold and as a subset of $X_{0}$.

Definition 7. For a pair of points $x, y$ in $X_{0}<X$, the following are equivalent.

a. $d\left(x ; X, X_{0}\right)=d\left(y ; X, X_{0}\right)=i$ and $x$ and $y$ lie in the same component of $I^{i}\left(X, X_{0}\right)-I^{i-1}\left(X, X_{0}\right)$.

b. There exists an ambient isotopy $H:\left(X, X_{0}\right) \times I \rightarrow\left(X, X_{0}\right) \times I$ such that $H_{0}=\mathrm{id}_{x}$ and $H(x, 1)=(y, 1)$.

If these conditions hold we will write $x \sim y$ in $\left(X, X_{0}\right)$.

Proof of the equivalence. $\mathrm{b} \rightarrow \mathrm{a}$. Let $d\left(x ; X, X_{0}\right)=i$, then $t \leadsto H(x, t)$ is a path in $I^{i}\left(X, X_{0}\right)-I^{i-1}\left(X, X_{0}\right)$ joining $x$ to $y$.

$\mathrm{a} \rightarrow \mathrm{b}$. For $x \in I^{i}\left(X, X_{0}\right)-I^{i-1}\left(X, X_{0}\right)$, let $f: \Delta^{i} *\left(Y, Y_{0}\right) \rightarrow\left(X, X_{0}\right)$ be an embedding with $f^{-1}\left(X_{0}\right)=\Delta^{i} * Y_{0}, f\left(\Delta^{i} * Y-\partial \Delta^{i} * Y\right)$ open in $X$ and $f^{-1}(x) \in$ Int $\Delta^{i}$.

If $d \in$ Int $\Delta^{i}$, let $k: \Delta^{i} \rightarrow \Delta^{i}$ be a homeomorphism rel $\partial \Delta^{i}$ with $k\left(f^{-1}(x)\right)=d$. $k * 1_{Y}$ is a self-homeomorphism of $\Delta^{i} *\left(Y, Y_{0}\right)$, rel $\partial \Delta^{i} * Y$ and extending $k$. It is isotopic to the identity on $\Delta^{i} *\left(Y, Y_{0}\right)$ rel $\partial \Delta^{i} * Y$ by the Alexander trick.

Hence, if $\bar{k}:\left(X, X_{0}\right) \cong\left(X, X_{0}\right)$ is defined to be $f\left(k * 1_{Y}\right) f^{-1}$ on $f\left(\Delta^{i} * Y\right)$ and the identity elsewhere then $\bar{k}$ is ambient isotopic to the identity and $\bar{k}(x)=f(d)$.

Thus, $X$ ambient isotopes to any other point of $f\left(\right.$ Int $\left.\Delta^{i}\right)$, which is a neighborhood of $X$ in $I^{i}\left(X, X_{0}\right)-I^{i-1}\left(X, X_{0}\right)$ as we saw in the proof of Theorem 6. 
It follows that the set of points to which $x$ will ambient isotope is open and closed in $I^{i}\left(X, X_{0}\right)-I^{i-1}\left(X, X_{0}\right)$ and since $y$ is in the same component of $I^{i}\left(X, X_{0}\right)$ $-I^{i-1}\left(X, X_{0}\right)$ as $x, x$ ambient isotopes to $y$.

REMARK. It is clear that if $x \sim y$ in $\left(X, X_{0}\right)$ then $\operatorname{Lk}\left(x ; X, X_{0}\right) \cong \operatorname{Lk}\left(y ; X, X_{0}\right)$. The embedding of Lemma 5 is usually obtained, as in the proof that $\mathrm{a} \rightarrow \mathrm{b}$ in Definition 1, by coning an isomorphism of the link of a point with a suspension. To get a stronger hold on the simplicial situation, we must examine the intrinsic properties of suspensions.

Lemma 8. Let $\left(X, X_{0}\right)=S^{n-1} *\left(Y, Y_{0}\right)$ and assume that $\left(X, X_{0}\right)$ is not an $(n+1)$ fold suspension, then

$$
S^{n-1}=I^{n-1}\left(X, X_{0}\right)=\left\{x \in X_{0}: \Sigma \operatorname{Lk}\left(x ; X, X_{0}\right) \cong\left(X, X_{0}\right)\right\} .
$$

Proof. Clearly, we have

$$
S^{n-1} \subset\left\{x \in X_{0}: \Sigma \operatorname{Lk}\left(x ; X, X_{0}\right) \cong\left(X, X_{0}\right)\right\} \subset I^{n-1}\left(X, X_{0}\right)
$$

where the second inequality follows from the assumption that $\left(X, X_{0}\right)$ is not an $(n+1)$-fold suspension.

But $I^{n-1}\left(X, X_{0}\right) \subset S^{n-1}$, for if $x \in S^{n-1} * Y_{0}-S^{n-1}$, Lk $\left(x ; X, X_{0}\right)$ is an $n$-fold suspension and hence $x \notin I^{n-1}\left(X, X_{0}\right)$.

As a corollary, we have the following result of Armstrong and Morton, [ $\left.\mathbf{A}_{1}\right]$ and $[\mathbf{M}]$.

Lemma 9. If $\Sigma^{r}\left(X, X_{0}\right) \cong \Sigma^{n}\left(Y, Y_{0}\right), r \leqq n$, then $\left(X, X_{0}\right) \cong \Sigma^{n-r}\left(Y, Y_{0}\right)$.

Proof. First we assume that $n$ is maximal, i.e. that $\Sigma^{n}\left(Y, Y_{0}\right)$ is not an $(n+1)$-fold suspension and prove the result in this special case by induction on $r$.

For $r=0$, the result is clear.

Now assume we are given a homeomorphism

$$
h: S^{r-1} *\left(X, X_{0}\right) \rightarrow S^{n-1} *\left(Y, Y_{0}\right) \text {. }
$$

Let $x \in S^{r-1}$, then

$$
\Sigma^{r-1}\left(X, X_{0}\right) \cong \operatorname{Lk}\left(x ; S^{r-1} *\left(X, X_{0}\right)\right) \cong \operatorname{Lk}\left(h(x) ; S^{n-1} *\left(Y, Y_{0}\right)\right) .
$$

By Lemma $8, h(x) \in S^{n-1}$ and hence

$$
\operatorname{Lk}\left(h(x) ; S^{n-1} *\left(Y, Y_{0}\right)\right) \cong \Sigma^{n-1}\left(Y, Y_{0}\right) \text {. }
$$

Note that since $\Sigma^{n}\left(Y, Y_{0}\right)$ was not an $(n+1)$-fold suspension, $\Sigma^{n-1}\left(Y, Y_{0}\right)$ is not an $n$-fold suspension, and so the inductive hypothesis of the special case applies and $\left(X, X_{0}\right) \cong \Sigma^{n-r}\left(Y, Y_{0}\right)$.

Now if $n$ is not maximal, $\Sigma^{n}\left(Y, Y_{0}\right) \cong \Sigma^{m}\left(Z, Z_{0}\right)$ where $m$ is maximal. By two applications of the special case result,

$$
\Sigma^{n-r}\left(Y, Y_{0}\right) \cong \Sigma^{n-r}\left(\Sigma^{m-n}\left(Z, Z_{0}\right)\right) \cong \Sigma^{m-r}\left(Z, Z_{0}\right) \cong\left(X, X_{0}\right)
$$


Corollary 10. $\Sigma^{n}\left(X, X_{0}\right) \cong \Sigma^{n}\left(Y, Y_{0}\right)$ implies $\left(X, X_{0}\right) \cong\left(Y, Y_{0}\right)$.

Definition 11. For a suspension pair $\left(X, X_{0}\right)$ (resp. a suspension $\left.X\right)$, we define the intrinsic sphere $S\left(X, X_{0}\right)$ (resp. $\left.S(X)\right)$, to be

$$
\begin{aligned}
S\left(X, X_{0}\right) & =\left\{x \in X_{0}: \Sigma \operatorname{Lk}\left(x ; X, X_{0}\right) \cong\left(X, X_{0}\right)\right\} \\
S(X) & =S(X, X)=\{x \in X: \Sigma \operatorname{Lk}(x ; X) \cong X\},
\end{aligned}
$$

and if a compact pair $\left(X, X_{0}\right)$ is not a suspension we define $S\left(X, X_{0}\right)=\{0\}$.

LEMMA 12. For any compact pair $\left(X, X_{0}\right)$ there is a homeomorphism $\left(X, X_{0}\right)$ $\cong S\left(X, X_{0}\right) *\left(Y, Y_{0}\right)$ rel $S\left(X, X_{0}\right)$, with $\left(Y, Y_{0}\right)$ unique up to homeomorphism and not a suspension.

Proof. If $\left(X, X_{0}\right)$ is not a suspension then let $\left(Y, Y_{0}\right)=\left(X, X_{0}\right)$. Otherwise let $\left(X, X_{0}\right)$ be an $n$-fold suspension but not an $(n+1)$-fold suspension. Then there exists a homeomorphism

$$
q:\left(X, X_{0}\right) \rightarrow S^{n-1} *\left(Y, Y_{0}\right)
$$

and $\left(Y, Y_{0}\right)$ is not a suspension. By Lemma $8, q\left(S\left(X, X_{0}\right)\right)=S^{n-1}$ so

$$
q^{-1} \mid S^{n-1}: S^{n-1} \cong S\left(X, X_{0}\right) \text {, }
$$

and joining with the identity on $Y$ and preceding by $q$ gives

$$
\left(X, X_{0}\right) \rightarrow S^{n-1} *\left(Y, Y_{0}\right) \rightarrow S\left(X, X_{0}\right) *\left(Y, Y_{0}\right)
$$

rel $S\left(X, X_{0}\right)$. Uniqueness of $\left(Y, Y_{0}\right)$ comes from Corollary 10.

REMARKS. If $f: S^{m} *\left(Y, Y_{0}\right) \rightarrow\left(X, X_{0}\right)$ is a homeomorphism then

$$
f\left(S^{m}\right) \subset S\left(X, X_{0}\right) .
$$

Hence, $\left(X, X_{0}\right)$ is a $\left(\operatorname{dim} S\left(X, X_{0}\right)+1\right)$ fold suspension and not a $\left(\operatorname{dim} S\left(X, X_{0}\right)+2\right)$ fold suspension.

Thus, if $x \in X_{0}$, we have the equation

$$
d\left(x ; X, X_{0}\right)=\operatorname{dim} S\left(\operatorname{Lk}\left(x ; X, X_{0}\right)\right)+1 .
$$

$S\left(X, X_{0}\right)$ is a subcomplex of any triangulation of $\left(X, X_{0}\right)$. For Lemma 8 implies that if $\left(X, X_{0}\right)$ is a suspension, $S\left(X, X_{0}\right)$ is precisely the first nonempty intrinsic skeleton.

We now examine the important phenomena of the points interior to a face of a simplex having the same intrinsic dimension as the points interior to the simplex:

Definition 13. Let $B=A C$ be a simplex of $K_{0}$ in the complex pair $\left(K, K_{0}\right)$, then the following conditions on $A$ and $B$ are equivalent:

a. $d\left(x ; K, K_{0}\right)=d\left(y ; K, K_{0}\right) \quad x \in$ Int $A, y \in$ Int $B$.

b. $\operatorname{Lk}\left(x ; K, K_{0}\right) \cong \operatorname{Lk}\left(y ; K, K_{0}\right) \quad x \in \operatorname{Int} A, y \in \operatorname{Int} B$.

c. $\mathrm{Lk}\left(A ; K, K_{0}\right) \cong \Sigma \dot{C} * \mathrm{Lk}\left(B ; K, K_{0}\right)$.

If these conditions hold we will say that $A$ is a nice face of $B$ in $\left(K, K_{0}\right)$. 
Proof of the equivalence. a $\rightarrow \mathrm{b}$. Let $d\left(y ; K, K_{0}\right)=i$, then $d\left(x ; K, K_{0}\right)=d\left(y ; K, K_{0}\right)$ $\rightarrow x \sim y$ in $\left(K, K_{0}\right)$ since Int $A$ lies in the closure of Int $B$. Hence, the links are isomorphic by the remark following Definition 7.

$\mathrm{b} \rightarrow \mathrm{a}$. Clear.

$\mathrm{b} \rightleftarrows \mathrm{c} . \operatorname{Lk}\left(x ; K, K_{0}\right) \cong \dot{A} \operatorname{Lk}\left(A ; K, K_{0}\right)$ and $\operatorname{Lk}\left(y ; K, K_{0}\right) \cong \dot{B} \operatorname{Lk}\left(B ; K, K_{0}\right)$. But,

$$
\dot{B}=(A \dot{C}+\dot{A C}) \cong \Sigma \dot{A} \dot{C}=\dot{A} \Sigma \dot{C}
$$

So $\mathrm{b} \rightleftarrows \mathrm{c}$ by Corollary 10 .

It is the explicit condition c, which relates the links of the two simplices that will be most useful. Let us examine the simplicial situation more closely.

Since $\operatorname{Lk}(B)=\mathrm{Lk}(C ; \operatorname{Lk}(A))$, it follows that $C * \operatorname{Lk}\left(B ; K, K_{0}\right)$ is actually a subcomplex pair of $\operatorname{Lk}\left(A ; K, K_{0}\right)$, and in fact, we have the usual stellar decomposition

$$
\operatorname{Lk}\left(A ; K, K_{0}\right)=C * \operatorname{Lk}\left(B ; K, K_{0}\right)+\left(P, P_{0}\right)
$$

where $\left(P, P_{0}\right) \cap C * \operatorname{Lk}\left(B ; K, K_{0}\right)=\dot{C} * \operatorname{Lk}\left(B ; K, K_{0}\right)$.

Thus, we let $\left(Q, Q_{0}\right)=\dot{C} * \operatorname{Lk}\left(B ; K, K_{0}\right)$ and identify $C * \operatorname{Lk}\left(B ; K, K_{0}\right)$ with $c\left(Q, Q_{0}\right)$, then we have $c\left(Q, Q_{0}\right) \subset \operatorname{Lk}\left(A ; K, K_{0}\right)$ with $c Q-Q$ open in $\operatorname{Lk}(A ; K, K)$. Now condition $\mathrm{c}$, is precisely the statement that $\mathrm{Lk}\left(A ; K, K_{0}\right) \cong \Sigma\left(Q, Q_{0}\right)$.

So the question arises: When can we find a homeomorphism $\operatorname{Lk}\left(A ; K, K_{0}\right)$ $\cong \Sigma\left(Q, Q_{0}\right)$, which takes $c\left(Q, Q_{0}\right)$ identically onto the "upper half" of $\Sigma\left(Q, Q_{0}\right)$ ? We will show that we can always "straighten" the homeomorphism on $C$, and in a first derived $\bmod B$, we can straighten the homeomorphism on all of $c Q$, as required.

First, if $A$ is a nice face of $B$, then

$$
c\left(Q, Q_{0}\right) \subset \mathrm{Lk}(A) \cong \Sigma\left(Q, Q_{0}\right)
$$

If $x$ is any point of $c S\left(Q, Q_{0}\right)-S\left(Q, Q_{0}\right), \mathrm{Lk}\left(x ; c\left(Q, Q_{0}\right)\right) \cong \mathrm{Lk}(x ; \mathrm{Lk}(A))$ and the former is easily seen to be homeomorphic to $\left(Q, Q_{0}\right)$.

Hence, $c S\left(Q, Q_{0}\right) \subset S(\operatorname{Lk}(A))$ and is in fact a face. That is,

$$
C * S\left(\operatorname{Lk}\left(B ; K, K_{0}\right)\right) \text { is a face of } S\left(\operatorname{Lk}\left(A ; K, K_{0}\right)\right) .
$$

Consider the composite homeomorphism

$$
\operatorname{Lk}(A) \stackrel{\phi}{\longrightarrow}\left(v_{0}+v_{1}\right) \dot{C} \mathrm{Lk}(B) \stackrel{\psi}{\longrightarrow}\left(v_{0}+v_{1}\right) \dot{C} S(\operatorname{Lk}(B)) * R
$$

where $\psi$ is the homeomorphism of Lemma 12 , and $R$ is some compact pair, which is a join complement of $S(\mathrm{Lk}(B))$ in $\mathrm{Lk}(B) . \psi$ can be chosen to be the identity on $\left(v_{0}+v_{1}\right) \dot{C}$

Let $c$ be an interior point of $C$. We would like to alter $\phi$ to get $\phi$ which on $C$ sends $\dot{C}$ identically to $\dot{C}$ and $c$ to $v_{0}$ and joins. 
We know that $\psi \phi(C * S(\operatorname{Lk}(B)))$ is a fact of $\left(v_{0}+v_{1}\right) \dot{C} S(\operatorname{Lk}(B))$. So we can extend the map

$$
\psi \phi(C * S(\operatorname{Lk}(B))) \stackrel{\phi^{-1} \psi^{-1}}{\longrightarrow} c \dot{C} * S(\operatorname{Lk}(B)) \stackrel{1}{\longrightarrow} v_{0} \dot{C} * S(\operatorname{Lk}(B)),
$$

to an auto-homeomorphism of $\left(v_{0}+v_{1}\right) \dot{C} * S(\operatorname{Lk}(B))$, called $\rho$. Define

$$
\phi=\psi^{-1}(\rho * 1) \psi \phi .
$$

This proves the first part of the following:

Lemma 14. Let $\left(K, K_{0}\right)$ be a complex pair with $B=A C$ a simplex of $K_{0}$, and $c \in$ Int $C$. Assume $A$ is a nice face of $B$ in $\left(K, K_{0}\right)$, then

a. There exists a homeomorphism

$$
\phi: \operatorname{Lk}\left(A ; K, K_{0}\right) \rightarrow(c+x) \dot{C} \operatorname{Lk}\left(B ; K, K_{0}\right)
$$

with $\phi \mid C$ mapping identically onto $c \dot{C}$.

b. If $\eta_{B} K$ is a first derived of $K \bmod B$, then there exists a homeomorphism:

$$
\phi: \operatorname{Lk}\left(A ; \eta_{B} K, \eta_{B} K_{0}\right) \rightarrow(c+x) \dot{C} \operatorname{Lk}\left(B ; \eta_{B} K, \eta_{B} K_{0}\right)
$$

with $\phi \mid C * \operatorname{Lk}\left(B ; \eta_{B} K, \eta_{B} K_{0}\right)$ the identity.

Proof. $\mathrm{a}$ is proved above, we now prove $\mathrm{b}$.

A simplex of $\mathrm{Lk}\left(A ; \eta_{B} K\right)$ is of the form $D_{0} \eta\left(A D_{0} D_{1}\right) \cdots \eta\left(A D_{0} D_{n}\right)$ with $D_{0}<C$ and $D_{1}<\cdots<D_{n}$. Mapping this simplex to $D_{0} \eta\left(D_{0} D_{1}\right) \cdots \eta\left(D_{0} D_{n}\right)$ gives a simplicial homeomorphism

$$
\operatorname{Lk}\left(A ; \eta_{B} K, \eta_{B} K_{0}\right) \rightarrow \eta_{C} \operatorname{Lk}\left(A ; K, K_{0}\right) .
$$

This homeomorphism takes $C \operatorname{Lk}\left(B ; \eta_{B}\left(K, K_{0}\right)\right)=C \operatorname{Lk}\left(C ; \operatorname{Lk}\left(A ; \eta_{B}\left(K, K_{0}\right)\right)\right)$ to $C \operatorname{Lk}\left(C ; \eta_{C} \operatorname{Lk}\left(A ; K, K_{0}\right)\right)=\operatorname{St}\left(C ; \eta_{C} \operatorname{Lk}\left(A ; K, K_{0}\right)\right)$. This is a regular neighborhood of $C \bmod C \dot{C}$ in $\mathrm{Lk}\left(A ; K, K_{0}\right)$.

Hence, $C \operatorname{Lk}\left(B ; \eta_{B}\left(K, K_{0}\right)\right)$ is a regular neighborhood of $C \bmod C$ in

$$
\operatorname{Lk}\left(A ; \eta_{B}\left(K, K_{0}\right)\right) \text {. }
$$

Using part a, define a homeomorphism

$$
\phi: \mathrm{Lk}\left(A ; \eta_{B}\left(K, K_{0}\right)\right) \rightarrow(c+x) \dot{C} \mathrm{Lk}\left(B ; \eta_{B}\left(K, K_{0}\right)\right)
$$

rel $C$. By uniqueness of regular neighborhoods there exists a homeomorphism:

$$
k:(c+x) \dot{C} \mathrm{Lk}(B) \rightarrow(c+x) \dot{C} \mathrm{Lk}(B)
$$

such that $k \mid c \dot{C}=$ identity and $k \phi(C * \mathrm{Lk}(B))=c \dot{C} \mathrm{Lk}(B)$. In particular,

$$
k \phi(\dot{C} * \operatorname{Lk}(B))=\dot{C} \operatorname{Lk}(B) .
$$

Finally, define

$$
\bar{k}:(c+x) \dot{C} \mathrm{Lk}(B) \rightarrow(c+x) \dot{C} \operatorname{Lk}(B)
$$

to be $(k \phi)^{-1}$ on $c \dot{C} \mathrm{Lk}(B)$ and extended by coning with $x \rightarrow x$ over $x \dot{C} \mathrm{Lk}(B)$. 
The required homeomorphism is $k k \phi$.

From the first part of the above we get a useful "local" version of our homogeneity results.

CoRollary 15. Let $\left(K, K_{0}\right)$ be a complex pair with $A$ a nice face of $B=A C$ in $\left(K, K_{0}\right)$. Let $b \in$ Int $B$. There exists a homeomorphism

$$
f: \mathrm{St}\left(A ; K, K_{0}\right) \rightarrow \mathrm{St}\left(A ; K, K_{0}\right) \operatorname{rel} A \operatorname{Lk}(A, K)
$$

and such that $f(B)=b \dot{A} C$.

Proof. Let $\phi$ be a homeomorphism

$$
\operatorname{Lk}(A) \rightarrow(c+x) \dot{C} \mathrm{Lk}(B)
$$

rel $c C$. Join with the identity on $A$ to extend $\phi$ to

$$
\phi: \mathrm{St}(A) \rightarrow(c+x) A \dot{C} \mathrm{Lk}(B) .
$$

Let $q:(c+x) A \dot{C} \rightarrow(c+x) A \dot{C}$ be a homeomorphism rel $(c+x) \dot{A} \dot{C}=\partial(c+x) A \dot{C}$ and mapping $c A C$ to $c b A \dot{C}$.

Define $f=\phi^{-1}(q * 1) \phi$.

RemarK. By the Alexander trick $f$ is isotopic to the identity $\operatorname{rel} A \operatorname{Lk}(A ; K)$.

We now relate the intrinsic skeleton and the links of simplices.

Lemma 16. Let $\left(K, K_{0}\right)$ be a complex pair $B=A C \in K_{0}, x \in$ Int $B$ and $y \in \operatorname{Int} C$, then

a. $d\left(x ; K, K_{0}\right)=\operatorname{dim} S\left(\operatorname{Lk}\left(B ; K, K_{0}\right)\right)+\operatorname{dim} B+1$.

b. $d\left(x ; K, K_{0}\right)=d\left(y ; \operatorname{Lk}\left(A ; K, K_{0}\right)\right)+\operatorname{dim} A+1$.

Proof. a. $S\left(\operatorname{Lk}\left(x ; K, K_{0}\right)\right)=\dot{B} * S\left(\operatorname{Lk}\left(B ; K, K_{0}\right)\right)$. So

$d\left(x ; K, K_{0}\right)=\operatorname{dim} S\left(\operatorname{Lk}\left(x ; K, K_{0}\right)\right)+1=\operatorname{dim} S\left(\operatorname{Lk}\left(B ; K, K_{0}\right)\right)+\operatorname{dim} B+1$.

b. By two applications of a, using $\operatorname{Lk}(C ; \operatorname{Lk}(A))=\operatorname{Lk}(B)$ :

$$
\begin{aligned}
d\left(x ; K, K_{0}\right) & =\operatorname{dim} S(\operatorname{Lk}(B))+\operatorname{dim} B+1, \\
d(y ; \operatorname{Lk}(A)) & =\operatorname{dim} S(\operatorname{Lk}(C ; \operatorname{Lk}(A)))+\operatorname{dim} C+1, \\
d\left(x ; K, K_{0}\right) & =d(y ; \operatorname{Lk}(A))+\operatorname{dim} B-\operatorname{dim} C .
\end{aligned}
$$

Finally, $\operatorname{dim} B-\operatorname{dim} C=\operatorname{dim} A+1$.

Corollary 17. Let $\left(K, K_{0}\right)$ be a complex pair and let $A \in K_{0}$ and

$$
C_{0}, C_{1} \in \operatorname{Lk}\left(A ; K_{0}\right) \text {. }
$$

Then

a. $A$ is a nice face of $A C_{0}$ in $\left(K, K_{0}\right)$ iff $C_{0} \in S\left(\operatorname{Lk}\left(A ; K, K_{0}\right)\right)$.

b. $A C_{0}$ is a nice face of $A C_{1}$ in $\left(K, K_{0}\right)$ iff $C_{0}$ is a nice face of $C_{1}$ in $\mathrm{Lk}\left(A ; K, K_{0}\right)$.

Proof. a. Let $x \in \operatorname{Int} A, y \in \operatorname{Int} A C_{0}$ and $z \in \operatorname{Int} C_{0}$. By Lemma 16a. $d\left(x ; K, K_{0}\right)$ $=\operatorname{dim} S\left(\operatorname{Lk}\left(A ; K, K_{0}\right)\right)+\operatorname{dim} A+1$. By Lemma $16 \mathrm{~b}$.

$$
d\left(y ; K, K_{0}\right)=d\left(z ; \operatorname{Lk}\left(A ; K, K_{0}\right)\right)+\operatorname{dim} A+1 .
$$


$A$ is a nice face of $A C_{0}$ iff $d\left(x ; K, K_{0}\right)=d\left(y ; K, K_{0}\right)$ iff $d\left(z ; \operatorname{Lk}\left(A ; K, K_{0}\right)\right)$ $=\operatorname{dim} S\left(\operatorname{Lk}\left(A ; K, K_{0}\right)\right)$ iff (by Lemma 8$) z \in S\left(\operatorname{Lk}\left(A ; K, K_{0}\right)\right)$.

b. From Lemma 16a, since Lk $\left(A C_{i} ; K, K_{0}\right)=\operatorname{Lk}\left(C_{i} ; \operatorname{Lk}\left(A ; K, K_{0}\right)\right)$ for $i=0,1$. Hence, a fortiori, $\operatorname{dim} S\left(\operatorname{Lk}\left(A C_{i}\right)\right)=\operatorname{dim} S\left(\operatorname{Lk}\left(C_{i} ; \operatorname{Lk}(A)\right)\right)$.

Corollary 18. Let $\left(K, K_{0}\right)$ be a complex pair and $A$ a $j$ simplex of $K_{0}$, then for $j \leqq i$

$$
\operatorname{Lk}\left(A ; I^{i}\left(K, K_{0}\right)\right)=I^{i-j-1}\left(\operatorname{Lk}\left(A ; K, K_{0}\right)\right) .
$$

Proof. This is just a restatement of Lemma $16 \mathrm{~b}$.

We should note what Lemma 16 and this corollary do and do not say. If $C \in \operatorname{Lk}\left(A ; K_{0}\right)$ and $x \in \operatorname{Int} A C, y \in \operatorname{Int} C$, then Lemma $16 \mathrm{~b}$ relates $d\left(x ; K, K_{0}\right)$ and $d\left(y ; \mathrm{Lk}\left(A ; K, K_{0}\right)\right)$. We would like to in turn conclude something about $d\left(y ; K, K_{0}\right)$. Since $C$ is a face of $A C, d\left(y ; K, K_{0}\right) \leqq d\left(x ; K, K_{0}\right)$ and hence we can conclude

$$
d\left(y ; K, K_{0}\right) \leqq d\left(y ; \operatorname{Lk}\left(A ; K, K_{0}\right)\right)+\operatorname{dim} A+1 .
$$

Furthermore, it is easy to construct examples to show that equality need not hold. For equality to hold it is clearly necessary and sufficient that $d\left(y ; K, K_{0}\right)$ $=d\left(x ; K, K_{0}\right)$, i.e. that $C$ be a nice face of $A C$ in $\left(K, K_{0}\right)$. Since this comes up in another context we mention the following special case when equality does hold:

Lemma 19. Let $\left(K, K_{0}\right)$ be a complex pair and $B=A C \in K_{0}$. For

$$
\begin{gathered}
y \in \operatorname{Lk}\left(A ; \eta_{B} K_{0}\right)-C, \\
d\left(y ; K, K_{0}\right)=d\left(y ; \operatorname{Lk}\left(A ; \eta_{B} K, \eta_{B} K_{0}\right)\right)+\operatorname{dim} A+1 .
\end{gathered}
$$

Proof. $y \in$ Int $D_{0} \eta\left(A D_{0} D_{1}\right) \cdots \eta\left(A D_{0} D_{n}\right)$ with $D_{0}<C, D_{1}<\cdots<D_{n} \neq 0, D_{i} \in K_{0}$ $i=0, \ldots, n$.

Int $D_{0} \eta\left(A D_{0} D_{1}\right) \cdots \eta\left(A D_{0} D_{n}\right) \cup$ Int $A D_{0} \eta\left(A D_{0} D_{1}\right) \cdots \eta\left(A D_{0} D_{n}\right) \subset$ Int $A D_{0} D_{n}$.

Hence, $D_{0} \eta\left(A D_{0} D_{1}\right) \cdots \eta\left(A D_{0} D_{n}\right)$ is a nice face of $A D_{0} \eta\left(A D_{0} D_{1}\right) \cdots \eta\left(A D_{0} D_{n}\right)$ in $\eta_{B}\left(K, K_{0}\right)$ and equality holds by the above remarks.

Lemma 20. Let $\left(X, X_{0}\right)$ be a compact polyhedral pair, then for $i \geqq 0$,

$$
\Sigma I^{i}\left(X, X_{0}\right)=I^{i+1}\left(\Sigma\left(X, X_{0}\right)\right) \text {. }
$$

Proof. Since $\Sigma S\left(X, X_{0}\right)=S\left(\Sigma\left(X, X_{0}\right)\right)$, the result is true for the minimal nonempty intrinsic skeleton, if $\left(X, X_{0}\right)$ is a suspension. If $\left(X, X_{0}\right)$ is not a suspension, then $I^{0}\left(\Sigma\left(X, X_{0}\right)\right)=$ suspension vertices. In either case, this takes care of the suspension vertices.

If $y=t v+(1-t) x$, with $v$ a suspension vertex, $0 \leqq t<1$ and $x \in X_{0}$,

$$
d\left(y ; \Sigma\left(X, X_{0}\right)\right)=d\left(x ; X, X_{0}\right)+1 .
$$

REMARK. As we saw in the proof, if $\left(X, X_{0}\right)$ is not a suspension then $I^{0}\left(\Sigma\left(X, X_{0}\right)\right)$ $=$ suspension vertices $=\Sigma\{0\}$, so if $\left(X, X_{0}\right)$ is not a suspension, we interpret $I^{-1}\left(X, X_{0}\right)=\{0\}$, which fits with the corresponding convention of Definition 11 . 
We obtain the following amusing corollary,

Corollary 21. Let $X$ be a polyhedron, then $I^{j}\left(X, I^{i}(X)\right)=I^{j}(X)$ for $j \leqq i$.

Proof. Let $x \in I^{i}(X)$, then by Corollary 18 ,

$$
\operatorname{Lk}\left(x ; X, I^{i}(X)\right)=\left(\operatorname{Lk}(x ; X), I^{i-1}(\operatorname{Lk}(x ; X))\right) .
$$

If $d(x ; X)=j$, then $\operatorname{Lk}(x ; X) \cong \Sigma^{j} Y$, with $Y$ not a suspension, then by Lemma 20 (and the remark which follows for the case $i=j$ ),

$$
\operatorname{Lk}\left(x ; X, I^{i}(X)\right) \cong\left(\Sigma^{j} Y, \Sigma^{j} I^{i-j-1}(Y)\right) \cong \Sigma^{j}\left(Y, I^{i-j-1}(Y)\right)
$$

Since $Y$ does not desuspend, the pair does not and hence $d\left(x ; X, I^{i}(X)\right)=j$.

We now relate the absolute intrinsic skeleton with the ambient notion:

Definition 22. Let $\left(X, X_{0}\right)$ be a polyhedral pair, with $x \in X_{0}$, then $\left(X, X_{0}\right)$ is called locally unknotted at $x$ if $d\left(x ; X, X_{0}\right)=d\left(x ; X_{0}\right)$. If $\left(X, X_{0}\right)$ is locally unknotted at all points of $X_{0}$, we call $\left(X, X_{0}\right)$ a locally unknotted pair.

Local unknotting translates absolute conditions on $X_{0}$ into ambient conditions on the pair, and can be defined in terms of any of the notions we have introduced:

THEOREM 23. For a complex pair $\left(K, K_{0}\right)$, the following are equivalent:

a. $\left(K, K_{0}\right)$ is locally unknotted.

b. For all $A \in K_{0}, S\left(\operatorname{Lk}\left(A ; K, K_{0}\right)\right)=S\left(\operatorname{Lk}\left(A ; K_{0}\right)\right)$.

c. $A$ a nice face of $B$ in $K_{0}$ implies that $A$ is a nice face of $B$ in $\left(K, K_{0}\right)$.

d. $x \sim y$ in $K_{0}$ implies $x \sim y$ in $\left(K, K_{0}\right)$.

e. $I^{i}\left(K, K_{0}\right)=I^{i}\left(K_{0}\right)$ for all $i$.

Proof. $\mathrm{a} \rightarrow \mathrm{e} \rightarrow \mathrm{d} \rightarrow \mathrm{c}$ are clear. To complete the proof, note that $\mathrm{c} \rightarrow \mathrm{b}$ by Corollary $17 \mathrm{a}$ applied to $\left(K, K_{0}\right)$ and $K_{0} . \mathrm{b} \rightarrow$ a by Lemma $16 \mathrm{a}$ applied to $\left(K, K_{0}\right)$ and $K_{0}$.

We relate these notions to the usual definition of locally unknottedness for manifolds, by the lemma.

Lemma 24. a. A sphere pair $\left(S^{n}, S^{p}\right)$ is unknotted iff $S\left(S^{n}, S^{p}\right)=S^{p}$.

b. $A$ ball pair $\left(B^{n}, B^{p}\right)$ is unknotted iff $S\left(B^{n}, B^{p}\right)=\partial B^{p}$.

Proof. In each case unknottedness clearly implies the intrinsic sphere condition and conversely $\left(S^{n-p},\{0\}\right)$ and $\left(B^{n-p+1}, p\right)$ (with $p \in$ Int $B^{n-p+1}$ ) are unknotted and hence so are any suspensions of them.

Corollary 25. Let $\left(X, X_{0}\right)$ be a proper manifold pair, i.e. $\partial X \cap X_{0}=\partial X_{0}$, then $\left(X, X_{0}\right)$ is locally unknotted iff it is locally unknotted in the usual sense, i.e. $x \in X_{0}$ implies $\mathrm{Lk}\left(x ; X, X_{0}\right)$ is an unknotted ball or sphere pair.

Proof. From Lemma 24, and the equivalence of locally unknottedness with $S\left(\operatorname{Lk}\left(x ; X, X_{0}\right)\right) \cong S\left(\operatorname{Lk}\left(x ; X_{0}\right)\right)$. 
Remark. Note that $\left(X, I^{i}(X)\right)$ need not be locally unknotted. Theorem 23 and Corollary 21 imply that this is equivalent to $I^{j}\left(I^{i}(X)\right)=I^{j}(X)$ for all $j \leqq i$. It is easy to see that this need not be true, e.g. the wedge of two different dimensional spheres.

III. Homogeneous collapsing and regular neighborhoods. We begin by defining a general form of relative collapsing.

Definition 1. Let $X$ be a polyhedron, with $X^{\prime}, X_{0}<X$ and let $X_{0}^{\prime}=X^{\prime} \cap X_{0}$, then $X$ collapses to $X^{\prime}$ relative to $X_{0}$, or more simply $\left(X, X_{0}\right)$ collapses to $\left(X^{\prime}, X_{0}^{\prime}\right)$, denoted $\left(X \mid X_{0}\right) \searrow X^{\prime}$ if there exists a sequence of elementary collapses

$$
X=X^{n} \searrow X^{n-1} \searrow \cdots \searrow X^{0}=X^{\prime}
$$

with $\mathrm{Cl}\left(X^{i+1}-X^{i}\right)=B^{i}$ and $B^{i} \cap X^{i}=F^{i}$, a ball and face, as usual, satisfying the condition that $B^{i} \cap X_{0}<\mathrm{Cl}\left(\partial B^{i}-F^{i}\right)$.

In such a case, we will call the sequence $\left(X^{n}, X^{n-1}, \ldots, X^{0}\right)$ a collapse of $X$ to $X^{\prime}$ rel $X_{0}$, or of $\left(X, X_{0}\right)$ to $\left(X^{\prime}, X_{0}^{\prime}\right)$.

There is a corresponding simplicial definition:

Definition 2. Let $K$ be a complex, with $K^{\prime}, K_{0}<K$ and let $K_{0}^{\prime}=K_{0} \cap K^{\prime}$, then $K$ collapses simplicially to $K^{\prime}$ relative to $K_{0}$, or $\left(K, K_{0}\right)$ collapses simplicially to $\left(K^{\prime}\right.$, $\left.K_{0}^{\prime}\right)$ if there exists a simplicial collapse $\left(K^{n}, K^{n-1}, \ldots, K^{0}\right)$ of $K$ to $K^{\prime}$ which is relative to $K_{0}$ in the sense of Definition 1, i.e. $K^{i+1}=K^{i}+B^{i}+A^{i}$ and $K_{0} \cap B^{i}<A^{i}$. We denote this by $\left(K \mid K_{0}\right) \searrow^{s} K^{\prime}$.

REMARKS. In the simplicial case, there are two possibilities, which represent the two kinds of things that can occur: Either $K_{0} \cap B^{i}=A^{i}$, or $K_{0} \cap B^{i}<\partial A^{i}$. The first corresponds to a punch out from $K_{0}$ and the second is in a strong sense, "away from $K_{0} "$.

Any subdivision of a collapse of $X$ to $X^{\prime}$ relative to $X_{0}$ or of a simplicial collapse of $K$ to $K^{\prime}$ relative to $K_{0}$ is clearly still a collapse relative to $X_{0}$, or $K_{0}$ respectively. Also, any collapse of $X$ to $X^{\prime}$ rel $X_{0}$ is a collapse relative to any subpolyhedron of $X_{0}$.

If $X_{0}<X^{\prime}$, then for all $i$ in a relative collapse, $B_{i} \cap X_{0}<\partial F^{i}$ and the definition reduces to Cohen's definition of relative collapsing. In this case, of course, $X_{0}^{\prime}=X_{0}$.

We now relate the geometrical and simplicial notions using the following elegant proof due to Marshall Cohen.

LEMMA 3. Let $\Delta^{n}=a \Delta^{n-1}=a d \Delta^{n-1}$ (with $d \in$ Int $\Delta^{n-1}$ ) and let $r: \Delta^{n} \rightarrow a \Delta^{n-1}$ be the simplicial retraction sending $d$ to $a$. Let $\alpha \Delta^{n}$ be any subdivision of $\Delta^{n}$ such that $r: \alpha \Delta^{n} \rightarrow \alpha a \dot{\Delta}^{n-1}$ is simplicial. Then $\left(\alpha \Delta^{n} \mid \alpha \Delta^{n-1}\right) \searrow^{s} \alpha \dot{a} \Delta^{n-1}$.

Proof. Let $A$ be a simplex of $\alpha a \dot{\Delta}^{n-1}-\alpha \dot{\Delta}^{n-1} \cdot r^{-1}(A)$ is a convex cell of dimension $\operatorname{dim} A+1$, with $A$ as a face and $r^{-1}(A) \cap \Delta^{n-1}$ as a face. The simplices of $r^{-1}(A)-\left(A \cup r^{-1}(A)\right)$ are mapped by $r$ onto $A$. They are ordered by the line segment $r^{-1}(\hat{A})$ which joins $\hat{A}$ to $r^{-1}(\hat{A}) \cap \operatorname{Int} \Delta^{n-1}$. We remove $\operatorname{dim} A$ and $\operatorname{dim} A+1$ simplices of $r^{-1}(A)$ in pairs moving down $r^{-1}(\hat{A})$ from $r^{-1}(\hat{A}) \cap \operatorname{Int} \Delta^{n-1}$. This gives a collapse $r^{-1}(A) \searrow^{s} r^{-1}(\dot{A}) \cup A$. Furthermore, this collapse is 
relative to $\Delta^{n-1}$, or more precisely, $\left(r^{-1}(A) \cap \alpha \Delta^{n-1}\right)$. For if $B$ and $C$ with $B=b C$ are a pair of simplices removed during this collapse then $r B=r C=A$ and so there is a vertex $c$ of $C$ such that $r b=r c$ is a vertex of $A$, and hence $b \notin \dot{\Delta}^{n-1}$ and since it lies between $c$ and $r b$ on the segment $r^{-1} r b, b \notin$ Int $\Delta^{n-1}$ so $B \cap \alpha \Delta^{n-1}<C$.

Now order the simplices of $\alpha a \dot{\Delta}^{n-1}-\alpha \dot{\Delta}^{n-1}$ in decreasing order of dimension and in order, for each such simplex $A$, perform the above simplicial collapse $r^{-1}(A) \searrow^{s} A \cup r^{-1}(\dot{A})$, giving a collapse $\alpha \Delta^{n} \searrow^{s} \alpha a \dot{\Delta}^{n-1}$, which is clearly relative to $\alpha \Delta^{n-1}$.

Lemma 4. Let $\left(X \mid X_{0}\right) \searrow X^{\prime}$ and let $(K ; h)$ be a triangulation of $X$. Then there exists a subdivision $\alpha K$ such that $h^{-1}\left(X_{0}\right)$ and $h^{-1}\left(X^{\prime}\right)$ are subcomplexes of $\alpha K$ and $\left(\alpha K \mid h^{-1}\left(X_{0}\right)\right) \searrow^{s} h^{-1}\left(X^{\prime}\right)$.

Proof. Let $X=X^{n}, \ldots, X^{0}=X^{\prime}$ be a collapse of $X$ to $X^{\prime}$ rel $X_{0}$, and let $B^{i}=h^{-1}\left(\mathrm{Cl}\left(X^{i}-X^{i-1}\right)\right)$ and $F^{i}$ the face of $B^{i}$ complementary to $B^{i} \cap h^{-1}\left(X^{i-1}\right)$. So $B^{i} \cap h^{-1}\left(X_{0}\right) \subset F^{i}$.

Let $q^{i}:\left(\Delta_{i}, \Delta_{i}^{\prime}\right) \rightarrow\left(B^{i}, F^{i}\right)$ be a homeomorphism with $\Delta_{i}=a_{i} \Delta_{i}^{\prime}$. If $r^{i}: \Delta_{i} \rightarrow a_{i} \dot{\Delta}_{i}^{\prime}$ is the retraction of Lemma 3, then define $s^{i}: h^{-1}\left(X^{i}\right) \rightarrow h^{-1}\left(X^{i-1}\right)$ to be $q^{i} r^{i}\left(q^{i}\right)^{-1}$ on $B^{i}$ and the identity on $h^{-1}\left(X^{i-1}\right)$.

First, let $\beta K$ be a subdivision of $K$ so that $h^{-1}\left(X_{0}\right)$ and $h^{-1}\left(X^{i}\right) i=0, \ldots, n$ are subcomplexes of $\beta K$. Then subdivide to make the following diagram simplicial:

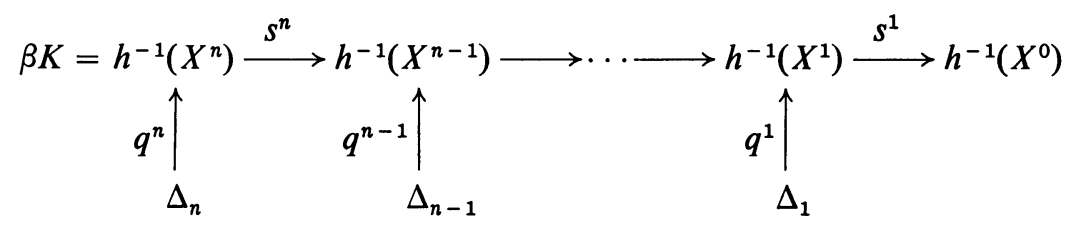

If $\alpha K$ is the subdivision of $\beta K$ obtained, then Lemma 3 implies that

$$
\left(\alpha K \mid h^{-1}\left(X_{0}\right)\right) \searrow^{s} h^{-1}\left(X^{\prime}\right) .
$$

RemarK. Notice that the simplicial collapse obtained is a subdivision of the original geometric collapse.

We now relate relative collapsing with intrinsic skeleta.

Definition 5. Assume that $X^{\prime}, X_{0}<X<Q_{0}<Q$, let $X=X^{n}, X^{n-1}, \ldots, X^{0}=X^{\prime}$ be a collapse of $X$ to $X^{\prime}$ rel $X_{0}$. Call this collapse homogenous (resp. weakly homogenous) with respect to $\left(Q, Q_{0}\right)$, if for each $i=0, \ldots, n-1, d\left(x ; Q, Q_{0}\right)$ is constant as $x$ varies over $X^{i+1}-X^{i}$ (resp. as $x$ varies over $X^{i+1}-\left(X^{i} \cup X_{0}\right)$ ). If there exists a collapse of $X$ to $X^{\prime}$ rel $X_{0}$ which is homogenous (resp. weakly homogenous) we will write $\left(X \mid X_{0}\right)_{h} \searrow X^{\prime}$ with respect to $\left(Q, Q_{0}\right)$ (resp. $\left(X \mid X_{0}\right)$ ${ }_{w} \searrow X^{\prime}$ with respect to $\left.\left(Q, Q_{0}\right)\right)$.

Note that $\left(X \mid X_{0}\right)_{n} \searrow X^{\prime}$ with resp. to $\left(Q, Q_{0}\right)$ does not imply that $d\left(x ; Q, Q_{0}\right)$ is constant as $x$ varies all over $X-X^{\prime}$. The intrinsic dimension of the cells which are removed may vary; $d\left(x ; Q, Q_{0}\right)$ is constant only on each cell. 
As usual we will write $\left(X \mid X_{0}\right)_{n} \downarrow X^{\prime}$ in $Q$ or $\left(X \mid X_{0}\right)_{w} \downarrow X^{\prime}$ in $Q$ for $\left(X \mid X_{0}\right)$ ${ }_{n} \succ X^{\prime}$ in $(Q, Q)$ or $\left(X \mid X_{0}\right)$ w $\downarrow X^{\prime}$ in $(Q, Q)$.

Note that if $X_{0}<X^{\prime}$, then $\left(X \mid X_{0}\right)_{n} \searrow X^{\prime}$ is equivalent to $\left(X \mid X_{0}\right)_{w} \searrow X^{\prime}$.

Assume that $\left(X \mid X_{0}\right)_{h} \downarrow X^{\prime}$ in $\left(Q, Q_{0}\right)$ and let $\left(K, K_{0} ; h\right)$ be any triangulation of $\left(Q, Q_{0}\right)$. Subdividing $K$ so that $h^{-1}(X)$ is a subcomplex, and then applying Lemma 4 , and the remark that follows it, and finally extending the resulting subdivision of $h^{-1}(X)$ to a subdivision of $K$, we obtain a subdivision $\left(\alpha K, \alpha K_{0} ; h\right)$ satisfying:

a. $h^{-1}(X), h^{-1}\left(X_{0}\right)$ and $h^{-1}\left(X^{\prime}\right)$ are subcomplexes of $\alpha K_{0}$.

b. $h^{-1}(X) \searrow^{s} h^{-1}\left(X^{\prime}\right)$ by a sequence of collapses of simplices $B^{i}=b^{i} A^{i}$ across faces $A^{i}$, satisfying:

1. $B^{i} \cap h^{-1}\left(X_{0}\right)<A^{i}$.

2. $A^{i}$ is a nice face of $B^{i}$ in $\left(\alpha K, \alpha K_{0}\right)$.

Similarly, if $\left(X \mid X_{0}\right){ }_{w} \succ X^{\prime}$ in $\left(Q, Q_{0}\right)$ and $\left(K, K_{0} ; h\right)$ is a triangulation of $\left(Q, Q_{0}\right)$, then there exists a subdivision $\left(\alpha K, \alpha K_{0} ; h\right)$ of $\left(Q, Q_{0}\right)$ satisfying:

a. $h^{-1}(X), h^{-1}\left(X_{0}\right)$ and $h^{-1}\left(X^{\prime}\right)$ are subcomplexes of $\alpha K_{0}$.

b. $h^{-1}(X) \searrow^{s} h^{-1}\left(X^{\prime}\right)$ by a sequence of collapses of simplices $B^{i}=b^{i} A^{i}$ across faces $A^{i}$, satisfying:

1. $B^{i} \cap h^{-1}\left(X_{0}\right)<A^{i}$.

2. If $B^{i} \cap h^{-1}\left(X_{0}\right)<A^{i}$ then $A^{i}$ is a nice face of $B^{i}$ in $\left(\alpha K, \alpha K_{0}\right)$.

In these simplicial situations, condition b1 says that the collapse is relative to $h^{-1}\left(X_{0}\right)$ and b2 says that the collapse is homogenous, or weakly homogenous.

Now after all these definitions, the collapsing theorems:

TheOREM 6. Assume $X_{0}<X<Q_{0}<Q$, and let $\left(V, V_{0}\right)$ be a regular neighborhood pair of $X \bmod X_{0}$ in $\left(Q, Q_{0}\right)$. Then if $\left(X \mid X_{0}\right)_{n} \searrow X^{\prime}$ with respect to $\left(Q, Q_{0}\right)$, then $\left(V, V_{0}\right)$ is a regular neighborhood pair of $X^{\prime} \bmod X^{\prime} \cap X_{0}$ in $\left(Q, Q_{0}\right)$.

Proof. We assume that everything is triangulated as above to obtain the situation: a complex pair $\left(K, K_{0}\right)$ with $K_{0}$ full in $K$ and subcomplexes $L, L^{\prime}, L_{0}$ of $K_{0}$ with $L_{0}$ full in $K_{0}$, and a simplicial collapse: $L=L^{n}, L^{n-1}, \ldots, L^{0}=L^{\prime}$ with $L^{i+1}$ $=L^{i}+A^{i}+B^{i}$ with $A^{i}$ a nice face of $B^{i}$ and $L_{0} \cap B^{i}<A^{i}$. If we prove that every $\left(K, K_{0}\right)$ neighborhood of $L^{i+1} \bmod L^{i+1} \cap L_{0}$ is one of $L^{i} \bmod L^{i} \cap L_{0}$, we are clearly finished by induction. So it suffices to prove the result across one simplicial collapse.

Let $L=L^{\prime}+A+B$ with $B=b A, L^{\prime} \cap B=b \dot{A}, L_{0} \cap B<A$ and $A$ a nice face of $B$ in $\left(K, K_{0}\right)$, where $L_{0}$ is a full subcomplex of $K$ and we can assume $L$ is a full subcomplex of $K$ by deriving mod $L$, if it were not. We show that every $\left(K, K_{0}\right)$ neighborhood of $L \bmod L_{0}$ is also a $\left(K, K_{0}\right)$ neighborhood of $L^{\prime} \bmod L_{0} \cap L^{\prime}$. By the uniqueness theorem for regular neighborhoods, it suffices to exhibit any regular neighborhood of $L \bmod L_{0}$ which is also a regular neighborhood of $L^{\prime} \bmod L_{0} \cap L^{\prime}$.

There are two cases, according to whether $B \cap L_{0}<\dot{A}$ or $B \cap L_{0}=A$.

(1) $B \cap L_{0}<\dot{A}$. Then $B \cap L_{0}$ is a proper face of $A$, by the fullness of $L_{0}$. 
Let $\left(H, H_{0}\right)=\mathrm{Lk}\left(B ; K, K_{0}\right)$. Let $\alpha K=\sigma_{b a} \sigma_{A} K$ where $\sigma_{A} K$ is stellar subdivision of $K$ at $A$ introducing a vertex $a \in \operatorname{Int} A$, and $\sigma_{b a} \sigma_{A} K$ is stellar subdivision of $\sigma_{A} K$ at $b a$ introducing a vertex $x \in \operatorname{Int} b a$.

$$
\alpha(B * H)=\sigma_{b a} \sigma_{A} b A * H=\sigma_{b a} b a \dot{A} * H=x(a+b) A * H=(\alpha B) * H .
$$

So

$$
\alpha\left(B *\left(H, H_{0}\right)\right)=\alpha \operatorname{St}\left(B ; K, K_{0}\right)=(\alpha B) *\left(H, H_{0}\right) .
$$

Define $L_{1}=L^{\prime}+x b \dot{A}$. This is a full subcomplex of $\alpha K$.

Assertion. There exists a derived $\eta \alpha K$ such that $N\left(L_{1}-L_{0} ; \eta \alpha\left(K, K_{0}\right)\right)$ is a regular neighborhood of $L^{\prime} \bmod L_{0}$ in $\left(K, K_{0}\right)$.

Note that as a first derived neighborhood $N\left(L_{1}-L_{0} ; \eta \alpha\left(K, K_{0}\right)\right)$ is by definition, a regular neighborhood of $L_{1} \bmod L_{0}$.

Assuming the above assertion, we are almost done, for by Corollary II.15 there exists a homeomorphism $f:\left(K, K_{0}\right) \rightarrow\left(K, K_{0}\right)$ supported by $\mathrm{St}^{\circ}(A ; K)$ such that $f(B)=x b \dot{A}$. Note that $f$ is the identity on $L^{\prime}$ and $L_{0}$ and $f(L)=L_{1}$. Hence,

$$
f^{-1} N\left(L_{1}-L_{0} ; \eta \alpha\left(K, K_{0}\right)\right)
$$

is a regular neighborhood of both $L \bmod L_{0}$ and $L^{\prime} \bmod L_{0}$ in $\left(K, K_{0}\right)$.

To prove the assertion, we parametrize $\sigma_{A} \mathrm{St}(B ; K)$. As above, $\sigma_{A}(B * H)$ $=\left(\sigma_{A} B\right) * H=b a \dot{A} * H$. Now let $\Delta=\left[e_{0}, e_{1}, e_{2}, e_{3}, e_{4}\right]$ be the standard four simplex, and define a simplicial map $p: \sigma_{A} B * H \rightarrow \Delta$ as the join of the following:

$$
\begin{array}{rlrl}
p\left(\dot{A} \cap L_{0}\right) & =e_{0}, & p(b)=e_{3}, \\
p\left(C\left(A \cap L_{0} ; \dot{A}\right)\right) & =e_{1}, & p(H)=e_{4}, \\
p(a) & =e_{2} .
\end{array}
$$

Note that the inverse of each vertex of $\Delta$ is a full subcomplex of $\sigma_{A} B * H$.

Furthermore, if we subdivide $\Delta: \sigma_{e_{2} e_{3}} \Delta$ at the point $e_{5}=p(x)$, then $p: \alpha B * H$ $\rightarrow \sigma_{e_{2} e_{3}} \Delta$ is simplicial, for $\alpha B * H=x(a+b) \dot{A} * H$ and $\sigma_{e_{2} e_{3}} \Delta=e_{5}\left(e_{2}+e_{3}\right) e_{0} e_{1} e_{4}$.

We will now define two derived subdivisions, one of $\Delta$ and one of $\sigma_{e_{2} e_{3}} \Delta$, called $\eta \Delta$ and $\bar{\eta} \sigma_{e_{2} e_{3}} \Delta$ respectively, satisfying:

(1) $\eta\left|e_{0} e_{1} e_{2} e_{4}+e_{0} e_{1} e_{3} e_{4}=\bar{\eta}\right| e_{0} e_{1} e_{2} e_{4}+e_{0} e_{1} e_{3} e_{4}$.

(2) $\left|N\left(e_{0} e_{1} e_{3}-e_{0} ; \eta \Delta\right)\right|=\left|N\left(e_{0} e_{1} e_{3} e_{5}-e_{0} ; \bar{\eta} \sigma_{e_{2} e_{3}} \Delta\right)\right|$.

Let $\bar{\eta}$ be any derived subdivision of $\sigma_{e_{2} e_{3}} \Delta$ and then pick $\eta \Delta$ to agree with $\bar{\eta}$ on $e_{0} e_{1} e_{2} e_{4}+e_{0} e_{1} e_{3} e_{4}$ and for $C$ any simplex (including 0$)$ of $e_{0} e_{1} e_{4}$, let $b\left(e_{2} e_{3} C\right)$ for $\eta \Delta$ be defined to equal $\bar{b}\left(e_{2} e_{5} C\right)$ of $\bar{\eta} \Delta$. (1) is clear and (2) is easily proved by looking at the complements of the two sets in $\Delta$ and seeing that they are made up of the same simplices.

Now, note that $\sigma_{A} K$ and $\alpha K$ agree outside of $\mathrm{St}^{\circ}(B ; K)$. Define derived subdivisions $\eta \sigma_{A} K$ and $\bar{\eta} \alpha K$ so that

(a) $\eta \sigma_{A}\left|K-\mathrm{St}^{\circ}(B ; K)=\bar{\eta} \alpha\right| K-\mathrm{St}^{\circ}(B ; K)$,

(b) $p: \eta \sigma_{A} B * H \rightarrow \eta \Delta$ and $p: \bar{\eta} \alpha B * H \rightarrow \bar{\eta} \sigma_{e_{2} e_{3}} \Delta$ are simplicial. 
Here (1) above is used to make (a) possible.

Now we claim that

$$
\left|N\left(L^{\prime}-L_{0} ; \eta \sigma_{A}\left(K, K_{0}\right)\right)\right|=\left|N\left(L_{1}-L_{0} ; \bar{\eta} \alpha\left(K, K_{0}\right)\right)\right|,
$$

which would prove the assertion since $L^{\prime}$ is full in $\sigma_{A} K$ and $L_{1}$ is full in $\alpha K$.

To prove the above equality note that the sets are clearly equal outside of $\mathrm{St}^{\circ}(B ; K)$. So it suffices to prove the equality of their intersection with $\mathrm{St}(B ; K)$, i.e. to prove

$$
\left|N\left(b \dot{A}-\dot{A} \cap L_{0} ; \eta \sigma_{A} B *\left(H, H_{0}\right)\right)\right|=\left|N\left(x b \dot{A}-\dot{A} \cap L_{0} ; \bar{\eta} \alpha B *\left(H, H_{0}\right)\right)\right| .
$$

But, by the parametrization Lemma [C; Lemma 2.14], these sets are respectively $\left(p, p \mid B * H_{0}\right)^{-1}\left|N\left(e_{0} e_{1} e_{3}-e_{0} ; \eta \Delta\right)\right|$ and $\left(p, p \mid B * H_{0}\right)^{-1}\left|N\left(e_{0} e_{1} e_{3} e_{5}-e_{0} ; \bar{\eta} \sigma_{e_{2} e_{3}} \Delta\right)\right|$ and are consequently equal by condition (2) above on $\eta \Delta$ and $\bar{\eta} \sigma_{e_{2} e_{3}} \Delta$.

This completes the proof of the first case.

(2) $B \cap L_{0}=A$. Let $L_{0}^{\prime}=L_{0} \cap L^{\prime}=L_{0}-A$. Again we assume that $L$ is full in $K$. Define $\alpha K=\sigma_{a b} \sigma_{A} K$ and $L_{1}=L^{\prime}+x b A$ as in the first case.

We reduce the proof of case (2), precisely as we did case (1) to a proof of the following:

ASSERTION. There exists a derived $\eta \alpha K$ such that $N\left(L_{1}-\left(L_{0}+x \dot{A}\right) ; \eta \alpha\left(K, K_{0}\right)\right)$ is a regular neighborhood of $L^{\prime} \bmod L_{0}^{\prime}$ in $\left(K, K_{0}\right)$.

Completing the proof of case (2) from the assertion uses the same homeomorphism $f$, and noting this time that $f\left(L_{0}\right)=L_{0}^{\prime}+x A$.

To prove the assertion, we parametrize again, this time to $\Delta=\left[e_{0} e_{1} e_{2} e_{3}\right]$ the standard three simplex. Define $p: \sigma_{A} B * H \rightarrow \Delta$ by joining

$$
\begin{aligned}
p(\dot{A}) & =e_{0}, & p(b) & =e_{2}, \\
p(a) & =e_{1}, & p(H) & =e_{3} .
\end{aligned}
$$

Let $p(x)=e_{4}$ and let $\sigma_{e_{1} e_{2}} \Delta$ introduce the vertex $e_{4}$, so that $p: \alpha B * H \rightarrow \sigma_{e_{1} e_{2}} \Delta$ is simplicial.

We now define derived subdivisions $\eta \Delta$ and $\bar{\eta} \sigma_{e_{1} e_{2}} \Delta$ to satisfy:

(1) $\eta\left|e_{0} e_{1} e_{3}+e_{0} e_{2} e_{3}=\bar{\eta}\right| e_{0} e_{1} e_{3}+e_{0} e_{2} e_{3}$.

(2) $\left|N\left(e_{0} e_{2}-e_{0} ; \eta \Delta\right)\right|=\left|N\left(e_{0} e_{2} e_{4}-e_{0} e_{4} ; \bar{\eta} \sigma_{e_{1} e_{2}} \Delta\right)\right|$.

Let $\bar{\eta}$ be any derived of $\sigma_{e_{1} e_{2}} \Delta$ and then pick $\eta \Delta$ to agree with $\bar{\eta}$ on $e_{0} e_{1} e_{3}+e_{0} e_{2} e_{3}$ and let $b\left(e_{1} e_{2}\right)=e_{4}, b\left(e_{1} e_{2} e_{0}\right)=\bar{b}\left(e_{0} e_{4}\right)$ of $\bar{\eta} \sigma_{e_{1} e_{2}} \Delta$ and $b\left(e_{1} e_{2} e_{3}\right)$ and $b\left(e_{0} e_{1} e_{2} e_{3}\right)$ $=\bar{b}\left(e_{2} e_{4} e_{3}\right)$ and $\bar{b}\left(e_{0} e_{2} e_{4} e_{3}\right)$ of $\bar{\eta} \sigma_{e_{1} e_{2}} \Delta$ respectively.

Define $\eta \sigma_{A} K$ and $\bar{\eta} \alpha K$ as in case (1) and we prove, precisely, as in case (1) the equality

$$
\left|N\left(L^{\prime}-L_{0}^{\prime} ; \eta \sigma_{A}\left(K, K_{0}\right)\right)\right|=\left|N\left(L_{1}-\left(L_{0}^{\prime}+x \dot{A}\right) ; \bar{\eta} \alpha\left(K, K_{0}\right)\right)\right| .
$$

This completes the proof of case (2) and hence of Theorem 6.

COROLlaRY 7. Let $X_{0}<X^{\prime}<X<Q$, and $V$ be a regular neighborhood of $X^{\prime} \bmod X_{0}$ in $V$, with $V$ a topological neighborhood of $X-X_{0}$. Then, if $\left(X \mid X_{0}\right)_{n} \backslash X^{\prime}$, then $V$ is a regular neighborhood of $X$ and $X_{0}$ in $Q$. 
Proof. Let $U$ be a regular neighborhood of $X \bmod X_{0}$ with $U-X_{0} \subset$ Int $V$ e.g. triangulate so that everything in the statement is a subcomplex with $X$ and $X_{0}$ full and then let $U=N\left(X-X_{0} ; \eta Q\right) \subset$ Int $V$ since $V$ is a topological neighborhood of $X-X_{0}$ and a subcomplex.

By Theorem 6, $U$ is a regular neighborhood of $X^{\prime} \bmod X_{0}$ and hence by [C; Proposition 7.3] $V$ is a regular neighborhood of $U \bmod X_{0}$ and hence by [C; Proposition 7.4] $V$ is a regular neighborhood of $X \bmod X_{0}$.

Now for the theorem associated with weakly homogenous collapsing:

THEOREM 8. Assume $X_{0}<X<Q_{0}<Q$, and let $\left(V, V_{0}\right)$ be a regular neighborhood of $X \bmod X_{0}$ in $\left(Q, Q_{0}\right)$. Then if $\left(X \mid X_{0}\right) w_{w} X^{\prime}$ with respect to $\left(Q, Q_{0}\right)$, there exists an embedding $f:\left(V, V_{0}\right) \rightarrow\left(V, V_{0}\right)$ with $f \mid X^{\prime}=$ identity and such that if $\left(U, U_{0}\right)$ $=f\left(V, V_{0}\right)$, then $\left(U, U_{0}\right)$ is a regular neighborhood of $X^{\prime} \cup X_{0} \bmod X_{0}$ in $\left(Q, Q_{0}\right)$ and $\left(\dot{U}, \dot{U}_{0}\right)=f\left(\dot{V}, \dot{V}_{0}\right)$.

Proof. We assume that everything is triangulated as in the remarks preceding Theorem 6, and so obtain the situation: a complex pair $\left(K, K_{0}\right)$ with $K_{0}$ full in $K$ and subcomplexes $L, L_{0}, L^{\prime}$ of $K_{0}$ with $L_{0}$ full in $K_{0}$, and a simplicial collapse $L=L^{n}, \ldots, L^{0}=L^{\prime}$, with $L^{i+1}=L^{i}+A^{i}+B^{i}$ with $L_{0} \cap B^{i}<A^{i}$ and $A^{i}$ a nice face of $B^{i}$ if $L_{0} \cap B^{i}<A^{i}$. If we prove that every $\left(K, K_{0}\right)$ neighborhood of $L^{i+1} \cup L_{0} \bmod L_{0}$ embeds in itself as required with image a regular neighborhood of $L^{i} \cup L_{0} \bmod L_{0}$, then the result follows by induction, by composing the embeddings. So again it suffices to prove the result across one simplicial collapse.

Let $L=L^{\prime}+A+B$ with $B=b A, L^{\prime} \cap B=b \dot{A}, L_{0} \cap B<A$ and if $L_{0} \cap B<A$, then $A$ is a nice face of $B . L_{0}$ is a full subcomplex of $K$ and we can again assume that $L$ is also full. As in Theorem 6, there are two cases:

(1) $L_{0} \cap B<A$ and $A$ is a nice face of $B$. Then Theorem 6 itself applies and any regular neighborhood of $L \bmod L_{0}$ is a regular neighborhood of $L^{\prime} \bmod L^{\prime} \cap L_{0}=L_{0}$ so we can let $f=$ identity, in this case.

(2) $L_{0} \cap B=A$. Note that as in Theorem 6, uniqueness of regular neighborhoods implies that we need only prove the existence of such an $f$ for one regular neighborhood of $L \cup L_{0} \bmod L_{0}$, since any other can be mapped to it by a homeomorphism of $\left(K, K_{0}\right)$ rel $L \cup L_{0}$.

As in the proof of Theorem 6 , let $\left(H, H_{0}\right)=\mathrm{Lk}\left(B ; K, K_{0}\right), \alpha K=\sigma_{a b} \sigma_{A} K$ and define $p: \sigma_{A} B * H \rightarrow \Delta=\left[e_{0} e_{1} e_{2} e_{3}\right]$, by joining

$$
\begin{aligned}
& p(\dot{A})=e_{0}, \quad p(b)=e_{2}, \\
& p(a)=e_{1}, \quad p(H)=e_{3} .
\end{aligned}
$$

Again, let $\sigma_{e_{1} e_{2}} \Delta$ introduce the vertex $e_{4}=p(x)$. Let $e_{5} \in \operatorname{Int} e_{2} e_{3}$. There exist deriveds $\eta \Delta$ and $\bar{\eta} \Delta$ satisfying

(1) $\eta\left|e_{0} \partial\left(e_{1} e_{2} e_{3}\right)=\bar{\eta}\right| e_{0} \partial\left(e_{1} e_{2} e_{3}\right)$.

(2) $\left|N\left(e_{0} e_{1} e_{2}-e_{0} e_{1} ; \eta \Delta\right)\right|=\left|e_{0} e_{1} e_{2} e_{5}\right|$ and $\left|\dot{N}\left(e_{0} e_{1} e_{2}-e_{0} e_{1} ; \eta \Delta\right)\right|=\left|e_{0} e_{1} e_{5}\right|$.

(3) $\left|N\left(e_{0} e_{2}-e_{0} ; \bar{\eta} \Delta\right)\right|=\left|e_{0} e_{4} e_{2} e_{5}\right|$ and $\left|\dot{N}\left(e_{0} e_{2}-e_{0} ; \bar{\eta} \Delta\right)\right|=\left|e_{0} e_{4} e_{5}\right|$. 
Now define derived $\eta \sigma_{A} K$ and $\bar{\eta} \sigma_{A} K$ to agree outside $\mathrm{St}^{\circ}(B ; K)$ and so that $p: \eta \sigma_{A} B * H \rightarrow \eta \Delta$ and $p: \bar{\eta} \sigma_{A} B * H \rightarrow \bar{\eta} \Delta$ are simplicial.

We will define a homeomorphism

$$
f:\left|N\left(L \cup L_{0}-L_{0} ; \eta \sigma_{A}\left(K, K_{0}\right)\right)\right| \rightarrow\left|N\left(L^{\prime} \cup L_{0}-L_{0} ; \bar{\eta} \sigma_{A}\left(K, K_{0}\right)\right)\right|
$$

taking $\dot{N}$ to $\dot{N}$ and the identity on $L^{\prime}$, which will prove the result.

To define $f$, note first that

$$
N\left(L \cup L_{0}-L_{0} ; \eta \sigma_{A} K\right)-\mathrm{St}^{\circ}(B ; K)-A=N\left(L^{\prime} \cup L_{0}-L_{0} ; \bar{\eta} \sigma_{A} K\right)-\mathrm{St}^{\circ}(B ; K),
$$

and

$$
\dot{N}\left(L \cup L_{0}-L_{0} ; \eta \sigma_{A} K\right)-\mathrm{St}^{\circ}(B ; K)-A=\dot{N}\left(L^{\prime} \cup L_{0}-L_{0} ; \bar{\eta} \sigma_{A} K\right)-\mathrm{St}^{\circ}(B ; K) .
$$

So define $f$ to be the identity on these sets. To complete the proof we must define $f$ on the intersection with $\mathrm{St}(B, K)$, i.e. we must extend $f$ to a homeomorphism

$$
N\left(B-A ; \eta \sigma_{A} B *\left(H, H_{0}\right)\right) \rightarrow N\left((b+a) \dot{A}-A ; \bar{\eta} \sigma_{A} B *\left(H, H_{0}\right)\right)
$$

taking boundary to boundary and the identity on the intersection with $b \dot{A} H$ (to extend the previous definition of $f$ ).

By the parametrization lemma, these sets are respectively,

$$
\left(p, p \mid B * H_{0}\right)^{-1}\left|e_{0} e_{1} e_{2} e_{5}\right| \text { and }\left(p, p \mid B * H_{0}\right)^{-1}\left|e_{0} e_{4} e_{2} e_{5}\right|,
$$

and the respective boundaries are the inverses under $\left(p, p \mid B * H_{0}\right)$ of $\left|e_{0} e_{1} e_{5}\right|$ and $\left|e_{0} e_{4} e_{5}\right|$.

Define $f: \sigma_{A} B * H \rightarrow \alpha B * H$ by $f(a)=x$ joined with the identity on $b \dot{A} * H$. We will prove that $f \mid N\left(B-A ; \eta \sigma_{A} B *\left(H, H_{0}\right)\right)$ is the required homeomorphism. To do this, it suffices to show that

$$
f p^{-1}\left|e_{0} e_{1} e_{2} e_{5}\right|=p^{-1}\left|e_{0} e_{4} e_{2} e_{5}\right| \text { and } f p^{-1}\left|e_{0} e_{1} e_{5}\right|=p^{-1}\left|e_{0} e_{4} e_{5}\right| .
$$

Define $f_{\Delta}: \Delta \rightarrow \sigma_{e_{1} e_{2}} \Delta$ by $f_{\Delta}\left(e_{1}\right)=e_{4}$ joined with the identity on $e_{0} e_{2} e_{3}$. Clearly, $p f=f_{\Delta} p$. Since $f_{\Delta}^{-1}\left(\left|e_{0} e_{4} e_{2} e_{5}\right|,\left|e_{0} e_{4} e_{5}\right|\right)=\left(\left|e_{0} e_{1} e_{2} e_{5}\right|,\left|e_{0} e_{1} e_{5}\right|\right)$ and since $f\left(\sigma_{A} B * H\right)$ contains $p^{-1}\left|e_{0} e_{4} e_{2} e_{5}\right|$, a little diagram chase proves that

$$
f p^{-1}\left(\left|e_{0} e_{1} e_{2} e_{5}\right|,\left|e_{0} e_{1} e_{5}\right|\right)=p^{-1}\left(\left|e_{0} e_{4} e_{2} e_{5}\right|,\left|e_{0} e_{4} e_{5}\right|\right) .
$$

Thus, $f$ defines a homeomorphism of a regular neighborhood of $L \cup L_{0} \bmod L_{0}$ onto a regular neighborhood of $L^{\prime} \cup L_{0} \bmod L_{0}$ within it. Proving case (2) and the theorem.

Corollary 9. Let $\left(X, X_{0}\right)$ be a polyhedral pair contained in a polyhedron $Q$. Let $V$ be a regular neighborhood of $X \bmod X_{0}$ in $Q$. If $\left(X \mid X_{0}\right){ }_{w} \backslash X^{\prime}$ in $Q$, then there are regular neighborhoods $U$ and $U^{\prime}$ of $X^{\prime} \bmod X_{0} \cap X^{\prime}$ in $Q$ contained in $V$ and there is an isotopy $H$ of $V$ into itself such that $H \mid V \times 0+U^{\prime} \times I=$ identity and such that $H_{1}(V)=U$. 
Proof. By Theorem 8, $V$ is homeomorphic rel $X^{\prime}$ to a regular neighborhood of $X^{\prime} \bmod X_{0} \cap X^{\prime}=X_{0}^{\prime}$. Hence, $V \cap X_{0}^{\prime}=\left(X_{0}^{\prime}\right)_{R}$ and $V$ supports a stellar triangulation as required by the hypothesis of part a of the stellar neighborhood theorem [C; Theorem 6.1(a)]. The conclusion is that of that theorem.

Before showing the various resolutions that these theorems give to the recognition problem for regular neighborhoods, we present the following equivalent to homogenous collapsing:

Lemma 10. Assume $X_{0}<X<Q_{0}<Q$ with $X-X_{0}$ finite dimensional, then $\left(X \mid X_{0}\right)$ ${ }_{n} \searrow X^{\prime}$ iff for all $0 \leqq i \leqq \operatorname{dim} X-X_{0}$ :

$$
\left(X \cap I^{i}\left(Q, Q_{0}\right) \mid X_{0} \cap I^{i}\left(Q, Q_{0}\right)\right) \searrow\left(X^{\prime} \cap I^{i}\left(Q, Q_{0}\right)\right) \cup\left(X \cap I^{i-1}\left(Q, Q_{0}\right)\right) .
$$

Proof. Let $I^{i}=I^{i}\left(Q, Q_{0}\right)$. The collapsing through intrinsic skeleta clearly implies homogenous collapsing since we can go from $X_{h} \downarrow X^{\prime} \cup\left(X \cap I^{i}\right)_{h} \searrow X^{\prime}$ by induction using the hypothesis for the inductive step.

To go the other way, we simply note that if we have a homogenous collapsing of $X$ to $X^{\prime}$, we can rearrange the collapses in descending order of intrinsic dimension, i.e. If $X^{i-1}+B^{i-1}=X^{i}$ and $X^{i+1}=X^{i}+B^{i+1}$ and $d\left(x ; Q, Q_{0}\right)$ is less for $x \in X^{i+1}-X^{i}$ than for $X^{i}-X^{i-1}$, then $B^{i+1} \cap X^{i}<X^{i-1}$ so we can first remove $B^{i-1}$ from $X^{i+1}$ and then remove $B^{i+1}$.

TheORem 11. Let $X_{0}<X<Q$, with $\mathrm{Cl}\left(X-X_{0}\right)=X_{R}$ compact, and let $V<Q$ be the closure of an open neighborhood of $X-X_{0}$, i.e. $V=\mathrm{Cl}$ Int $V$, with $X-X_{0} \subset \mathrm{Int} V$. Assume $V \cap X_{0}=\mathrm{Cl}\left(X-X_{0}\right) \cap X_{0}=\left(X_{0}\right)_{R}$, and let $\dot{V}=(\operatorname{Bdry} V) \cup\left(X_{0}\right)_{R}$. Then the following are equivalent:

a. $V$ is a regular neighborhood of $X \bmod X_{0}$ in $Q$.

b. $(V \mid \dot{V})_{n} \searrow(X)_{R}$.

c. $(V \mid \dot{V})_{w} \downarrow(X)_{R}$ and $\dot{V}-X_{0}$ is locally collared in $\mathrm{Cl}(Q-V)=Q-$ Int $V$.

d. $\left(V \mid\left(X_{0}\right)_{R}\right)_{n} \downarrow(X)_{R}$ and $\dot{V}-X_{0}$ is locally bicollared in $Q$.

e. $\mathrm{Cl}\left(V \cap I^{i}(Q)-X_{0}\right)$ is a regular neighborhood of

$$
\left(X \cap I^{i}(Q)\right) \cup\left(V \cap I^{i-1}(Q)\right) \bmod \left(X_{0} \cap I^{i}(Q)\right) \cup\left(\dot{V} \cap I^{i-1}(Q)\right)
$$

in $I^{i}(Q)$ for all $i$, and

$$
\left(\operatorname{Bdry}_{I^{i}(Q)} \mathrm{Cl}\left(V \cap I^{i}(Q)-X_{0}\right)\right) \cup\left(X_{0} \cap I^{i}(Q)\right)=\left(\dot{V} \cup X_{0}\right) \cap I^{i}(Q) .
$$

REMARK. All of the collapsing conditions $b-d$ have equivalent formulations including all of $X_{0}$. The equivalence comes from excision, and the assumption that $V \cap X_{0}=\left(X_{0}\right)_{R}$. The corresponding conditions are

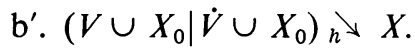

$\mathrm{c}^{\prime} .\left(V \cup X_{0} \mid \dot{V} \cup X_{0}\right)_{w} \searrow X+$ local collaring on the outside.

$\mathrm{d}^{\prime} .\left(V \cup X_{0} \mid X_{0}\right)_{n} \searrow X+$ local bicollaring. 
Proof. $a \rightarrow b$. Directly from the definition of regular neighborhoods, we have full subcomplexes $L, L_{0}$ of $K$, with $L-L_{0}$ finite and we wish to show that

$$
\left(N\left(L-L_{0} ; \eta K\right) \mid \dot{N}\left(L-L_{0} ; \eta K\right)\right)_{h} \backslash(L)_{R}
$$

in $K$. Note that by local finiteness $N\left(L-L_{0} ; \eta K\right)$ is a finite complex.

Every simplex of $N\left(L-L_{0} ; K\right)-L$ is of the form $A B C$ with $A \in\left(L_{0}\right)_{R}, B \in C\left(L_{0}, L\right)$ and $C \in \dot{N}\left((L)_{R} ; \dot{N}\left(L-L_{0} ; K\right)\right)$ [C; Proposition 2.9], with $B \neq 0 \neq C$. Order these simplices in descending order of dimension.

$\left(N\left(L-L_{0} ; \eta K\right), \dot{N}\left(L-L_{0} ; \eta K\right)\right) \cap A B C=(N(A B-A ; \eta A B C), \dot{N}(A B-A ; \eta A B C))$

from the parametrization lemma. By [C; Lemma 2.16] this is a ball and face, with complementary face

$$
N(A B-A, \eta \partial A B C)=N(A B-A ; \eta(B * \partial A C))=\bigcup_{i}\left(N\left(L-L_{0} ; \eta K\right) \cap A_{i} B C_{i}\right)
$$

for $A_{i} B C_{i} \leqq \partial A B C$. Thus, removing these pairs in decreasing order of dimension provides a collapse $N\left(L-L_{0} ; \eta K\right) \searrow(L)_{R}$ which is clearly rel $\dot{N}\left(L-L_{0} ; \eta K\right)$. Since the points removed in the collapse corresponding to $A B C$ all lie in the interior of $A B C$, the collapse is homogenous in $K$.

$\mathrm{a} \rightarrow \mathrm{c}$ and $\mathrm{d}$. Follows from $\mathrm{a} \rightarrow \mathrm{b}$ and [C; Theorem 5.2] which says that $\dot{V}-X_{0}$ is locally collared in $V$ and in $Q-$ Int $V$.

a $\rightarrow$ e. Triangulate the situation to obtain $L_{0}<L$ full subcomplexes of $K$ and $V=N\left(L-L_{0} ; \eta K\right)$. Recall that the intrinsic skeleta $I^{i}$ are subcomplexes of $K$. Let $V^{i}=\mathrm{Cl}\left(N\left(L-L_{0} ; \eta K\right) \cap I^{i}-L_{0}\right)$. By [C; Lemma 2.13], $V^{i}=N\left(L \cap I^{i}-L_{0} \cap I^{i} ; \eta I^{i}\right)$ for all $i$. Hence, $\left(V^{i}, V^{i-1}\right)$ is a regular neighborhood pair of $L \cap I^{i} \bmod L_{0} \cap I^{i}$ in $\left(I^{i}, I^{i-1}\right)$. This implies by [C; Proposition 7.10] that $V^{i}$ is a regular neighborhood, in $I^{i}$, of

$$
\left(L \cap I^{i}\right) \cup V^{i-1} \bmod \left(\operatorname{Bdry}_{I^{i-1}} V^{i+1}\right) \cup\left(L_{0} \cap I^{i}\right) .
$$

But $\left(L \cap I^{i}\right) \cup V^{i-1}=\left(L \cap I^{i}\right) \cup\left(V \cap I^{i-1}\right)$. And

$$
\begin{aligned}
\left(\operatorname{Bdry}_{I^{i-1}} V^{i-1}\right) \cup\left(L_{0} \cap I^{i}\right) & =\dot{N}\left(L \cap I^{i-1}-L_{0} \cap I^{i-1} ; \eta I^{i-1}\right) \cup\left(L_{0} \cap I^{i}\right) \\
& =\left(\dot{N}\left(L-L_{0} ; \eta K\right) \cap I^{i-1}\right) \cup\left(L_{0} \cap I^{i}\right) \\
& =\left(\dot{V} \cap I^{i-1}\right) \cup\left(L_{0} \cap I^{i}\right) .
\end{aligned}
$$

For in each of these equalities the only points where the varying terms change lie in $L_{0} \cap I^{i-1}$.

$\mathrm{b} \rightarrow \mathrm{a} . V$ is obviously a regular neighborhood of $V \cup X_{0} \bmod X_{0} \cup \dot{V}$ $\left(V \cap X_{0}=\left(X_{0}\right)_{R}\right.$ and $V=\mathrm{Cl}($ Int $V)$ ). So a follows from Theorem 6.

$\mathrm{c} \rightarrow \mathrm{a}$. As above, using Theorem 8 , we have $(V, \dot{V})$ is homeomorphic rel $X$ to a regular neighborhood of $X \bmod X_{0}$ and its boundary. Hence, $\dot{V}-X_{0}$ is locally bicollared and the stellar neighborhood theorem [C; Theorem 6.1b] implies that $V$ is a regular neighborhood of $X \bmod X_{0}$. 
$\mathrm{d} \rightarrow \mathrm{a}$. Triangulate and subdivide so that $X, X_{0}$ and $V$ are full subcomplexes of $Q$. The local bicollaring assumption implies that $\dot{V}-X_{0}$ is locally collared in $V$ and $Q-$ Int $V\left(Q-X_{0}=\left(Q-\left(V \cup X_{0}\right)\right) \cup\left(\dot{V}-X_{0}\right) \cup\left(\right.\right.$ Int $\left.\left.V-X_{0}\right)\right)$. Using this we can produce a homeomorphism $f: Q \rightarrow Q$ rel $X$ with $f V=N\left(V-X_{0} ; \eta Q\right)$, see for example the proof of [C; Theorem 6.1]. Theorem 6 implies that $N\left(V-X_{0} ; \eta Q\right)$ is a regular neighborhood of $X \bmod X_{0}$ in $Q$ and hence so is $V$.

$\mathrm{e} \rightarrow \mathrm{b}$. Let $V^{i}=\mathrm{Cl}\left(V \cap I^{i}-X_{0}\right)$. We have assumed the identity

$$
\left(\operatorname{Bdry}_{I^{i}} V^{i}\right) \cup\left(X_{0} \cap I^{i}\right)=\left(\dot{V} \cup X_{0}\right) \cap I^{i} .
$$

Then, since $a \rightarrow b^{\prime}$, the hypothesis of e gives

$$
\begin{aligned}
\left(V^{i} \cup\left(X_{0} \cap I^{i}\right) \cup\left(\dot{V} \cap I^{i-1}\right) \mid(\right. & \left.\left(\operatorname{Bdry}_{I^{i}} V^{i}\right) \cup\left(X_{0} \cap I^{i}\right) \cup\left(\dot{V} \cap I^{i-1}\right)\right) \\
& \searrow\left(X \cap I^{i}\right) \cup\left(V \cap I^{i-1}\right) .
\end{aligned}
$$

Apply the assumed identity, this becomes

$$
\left(\left(V \cup X_{0}\right) \cap I^{i} \mid\left(\dot{V} \cup X_{0}\right) \cap I^{i}\right) \searrow\left(X \cap I^{i}\right) \cup\left(V \cap I^{i-1}\right) .
$$

So Lemma 10 implies that $\left(V \cup X_{0} \mid \dot{V} \cup X_{0}\right)_{n} \downarrow X$.

REMARKS. The identity at the end of e is equivalent to the assumption $(V-\dot{V})$ $\cap I^{i}(Q)=$ Int $V^{i}-X_{0}$. Without this assumption $\mathrm{e} \rightarrow \mathrm{a}$. Let $Q$ be the upper half plane, $V=[(-2,0),(-1,1),(0,0)]+[(0,0),(1,1),(2,0)]$ and let $X=\{(-1,0)\}$ and $X_{0}=\varnothing$, then the conditions of e except for the identity are satisfied and yet $V$ is not a regular neighborhood of $X$.

There is an interesting example which shows that the local collaring conditions of $\mathrm{c}$ and $\mathrm{d}$ are necessary:

Let $\Delta_{1}=[(0,1),(0,-1),(1,0)]$ and $\Delta_{2}=[(0,0),(1 / 2,1 / 2),(1 / 2,-1 / 2)]$ in the plane $R^{2}$. Let $V=\Delta_{1} \times[0,1]+\Delta_{2} \times[-1,0]$ in the half space of $R^{3}=\{(x, y, z): x \geqq 0\}$ $=Q$. Let $X=(0,0,1 / 2)$ and $X_{0}=\varnothing$. One can check that $(V \mid \dot{V})_{w \downarrow} X$ and $V \downarrow \backslash X$ (by two different collapsings), but $V$ is not a regular neighborhood of $X$. In fact, $V \cap \partial Q$ is not a manifold.

We remark that the equivalence of a and $\mathrm{d}$ in the case $X_{0}=\varnothing$ is due to Stallings, by a rather different approach, see [S; Chapter VI].

If instead of condition e, we assume that $V^{i}$ is a regular neighborhood of $\left(X \cap I^{i}\right) \cup\left(V \cap I^{i-1}\right)=\left(X \cap I^{i}\right) \cup V^{i-1} \bmod \left(X_{0} \cap I^{i}\right) \cup\left(\operatorname{Bdry}_{I^{i-1}} V^{i-1}\right)$, then the identity follows by downward induction since, $x \in\left(\dot{V} \cap I^{i}\right)-\left(\operatorname{Bdry}_{I^{i}} V^{i} \cup X_{0}\right)$ implies $x \in \operatorname{Int} V^{i}$ and hence by hypothesis $x \in \operatorname{Int} V^{i+1}$, contradicting inductive hypothesis for $i+1$. Note that identity is clear for $i=\operatorname{dim} V$.

In the unlikely event that the reader is interested we mention a generalization of Theorems 6 and 8.

In our definition $\left(X \mid X_{0}\right)_{h} \searrow X^{\prime}$ in $\left(Q, Q_{0}\right)$ we assumed $X<Q_{0}$. To discard this assumption we can extend the previous definitions of homogenous and weakly homogenous collapsing using the old definitions as follows: Define $\left(X \mid X_{0}\right)_{h} \backslash X^{\prime}$ in $\left(Q, Q_{0}\right)$ to mean $\left(X \mid X_{0}\right)_{n \searrow} X^{\prime} \cup\left(X \cap Q_{0}\right)$ in $Q$ and $\left(X \cap Q_{0} \mid X_{0} \cap Q_{0}\right)$ 
${ }_{n} \searrow X^{\prime} \cap Q_{0}$ in $\left(Q, Q_{0}\right)$ (old definition). Similarly, we can extend the definition of $\left(X \mid X_{0}\right) w \downarrow X^{\prime}$ in $\left(Q, Q_{0}\right)$. Theorems 6 and 8 remain true as stated, deleting the assumption that $X, X_{0}<Q_{0}$ and using the new definitions. The proofs are the same but there are four cases, in each theorem, the two of Theorem 6 for collapses in $Q-Q_{0}$ and the two of Theorem 6 for collapses in $Q_{0}$. Similarly, for Theorem 8 .

Finally, we note that our solution of the "recognition problem" for regular neighborhoods, Theorem 11, has the unsatisfactory aspect of requiring $\mathrm{Cl}\left(X-X_{0}\right)$ to be compact. To eliminate this hypothesis, presumably one could replace collapsing conditions by the existence of some kind of "homogenously collapsible retraction", and construct a proof à la [C; Chapter 9] by using the retraction to reduce the problem to collapsing conditions on the dual cells, which are finite, and use Theorem 11 where Cohen uses Whitehead's shelling process. However, this would seem to require more attention than this outline suggests as the dual cells are cones, not balls in this case. Probably, a proof can be constructed using the results on cones, later in this paper, to generalize $a \rightleftarrows d$ to this context.

IV. Unknotting cone pairs. Given an embedding of a cone into a polyhedron: $f: a X \rightarrow Q$ and $V$ a regular neighborhood of $f(a X) \bmod f(X)$ in $Q$. We consider the problem of when $f: a X \rightarrow V$ looks like the inclusion of a subcone in a cone, i.e. when does there exist a homeomorphism $h: V \rightarrow a \dot{V}$ such that $h f=\bar{f}$ when $\bar{f}$ is defined as the coning of $f \mid X$, i.e. $a(f \mid X): a X \rightarrow a \dot{V}$. This is a much stronger condit ${ }^{\circ}$ on that the existence of a homeomorphism of pairs: $(V, f(a X)) \cong a(\dot{V}, f(X))$, for this homeomorphism preceded by $f$ need not equal $\bar{f}$, but will, in general, be $\bar{f}$ preceded by a homeomorphism $k: a X \rightarrow a X$ rel $X$. So this problem has two aspects: Unknotting the pair $(V, f(a X))$ and unknotting the homeomorphism $k$. The solution of the second is by far the more important and more difficult. In fact, it is an easy exercise to show using Theorem III.8, that $(V, f(a X)) \cong a(\dot{V}, f(X))$ iff there

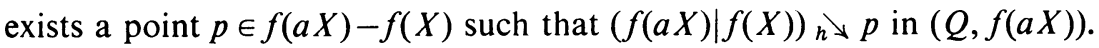

The importance of unknotting the embeddings of cones (rather than the set pairs) and the above version of unknotting is due to Lickorish [L] and this whole section is just a generalization of his Proposition 1, and in broad design mimics its proof.

Definition 1. Let $f: a X \rightarrow Q$ be an embedding. Call $f$ conewise homogenous, denoted $\mathrm{CH}$, if for each $x \in X, d(f(t a+(1-t) x) ; Q, f(a X))$ is constant for $0<t<1$.

LEMmA 2. Let $a\left(X, X_{0}\right)$ be a cone pair, then the inclusion $a X_{0} \subset a X$ is conewise homogenous.

Proof. If $x \in X_{0}$, then for any $0<t<1$ :

$$
\operatorname{Lk}\left(t a+(1-t) x ; a\left(X, X_{0}\right)\right) \cong \Sigma \operatorname{Lk}\left(x ; X, X_{0}\right),
$$

which is independent of $t$. 
REMARKS. It follows that conewise homogeneity is surely a necessary condition for unknotting an embedding as described above. The remainder of this section is devoted to showing that it is sufficient.

Note that for each $x \in X, d(f(t a+(1-t) x) ; f(a X))=d(t a+(1-t) x ; a X)=1$ $+d(x ; X)$ for $0<t<1$ and hence if $(Q, f(a X))$ is locally unknotted at every point of $f(a X)-f(X)$, then $f$ is conewise homogenous.

Since every triangulation of a cone has a conical subdivision [Z; Lemma 2.1], we can triangulate and subdivide an embedding $f: a X \rightarrow Q$ to obtain the simplicial situation:

$f: \gamma a K \rightarrow J$ a simplicial embedding with $\gamma a K$ a conical subdivision of $a K$ and $\gamma K=K$. We will call such an embedding $f: \gamma a K \rightarrow J$ a conical simplicial embedding.

We will need some properties of and notation about conical subdivisions which we now describe:

On a cone $a X$, there is a canonical projection $\pi: a X-a \rightarrow X$ given by

$$
\pi(t a+(1-t) x)=x
$$

for $t \neq 1$. This map is a "standard mistake" and is not p.l.; however, to say that $\gamma a K$ is a conical subdivision of $a K$ is precisely to say that if $A$ is a simplex of $\gamma a K-a$, then $\pi(A-a)$ is a simplex of $\gamma K$.

Let $\gamma a K$ be a conical subdivision with $\gamma K=K$. Then for $A \in \gamma a K-a$ we will denote by $\pi A$ the simplex $\pi(A-a)$ of $K$. For $A \in \gamma a K-a$, either $\operatorname{dim} A=\operatorname{dim} \pi A$, in which case we call $A$ horizontal, or $\operatorname{dim} A=\operatorname{dim} \pi A+1$, in which case we call $A$ vertical. If $B \in K$, then $\gamma a B-\gamma a \dot{B}$ consists of precisely those simplices $A$ such that $\pi A=B$, and the simplices of Int $\gamma a B$ are those $A$ such that $\pi A=B$ and $A \neq B$.

If $A$ is a horizontal simplex of $\gamma a B-\gamma a \dot{B}$, then the component of $a B-A$ which contains $a$, has precisely one vertical simplex with $A$ as a face. We will call this simplex, the simplex above $A$. If $A$ is a horizontal simplex of Int $\gamma a B$, then the component of $a B-A$ which intersects $K$ has precisely one vertical simplex with $A$ as a face. We will call this the simplex below $A$. Thus, if $A \in \gamma a K-a$ the simplex above $A$ lies in Int $\gamma a(\pi A)$ and if $A \notin K$ then the simplex below $A$ is also defined and lies in Int $\gamma a(\pi A)$.

Lemma 3. Let $f: \gamma a K \rightarrow J$ be a conical simplicial embedding. Then the following are equivalent:

a. $f$ is conewise homogenous.

b. If $B \in K$, then $d(f(x) ; J, f(a K))$ is constant for $x$ varying in Int $a B$.

c. If $A \in \gamma a K-(a \cup K)$ and $B$ is the simplex above or below $A$, then $f A$ is a nice face of $f B$ in $(J, f(\gamma a K))$.

Proof. Clearly, $\mathrm{c} \rightarrow \mathrm{b} \rightarrow \mathrm{a}$. We show that $\mathrm{a} \rightarrow \mathrm{c}$. If $x \in \operatorname{Int} \pi A=\pi B$, then the open cone line $\{t a+(1-t) x ; 0<t<1\}$ intersects Int $A$ and Int $B$. Since $f$ is $\mathbf{C H}$, $d(f(y) ; J, f(a K))$ is constant for $y$ along this line, so $f A$ is a nice face of $f B$. 
THEOREM 4. Let $f: a X \rightarrow Q$ be a conewise homogenous embedding. Let $V$ be $a$ regular neighborhood of $f(a X) \bmod f(X)$ in $Q$. Then there exists a homeomorphism $h: V \rightarrow a \dot{V}$ rel $\dot{V}$ such that $h f=\bar{f}$, where $\bar{f}$ is defined to be $a *(f \mid X)$.

Proof. We will inductively assume the theorem for $\operatorname{dim} X<n$ and prove it for $\operatorname{dim} X=n$.

Triangulate to obtain $f: \gamma a K \rightarrow J$ a conical simplicial embedding. There is a canonical simplicial collapse $(\gamma a K \mid K) \searrow a$, obtained by ordering the simplices of $K$ in descending order of dimension $\left\{D_{j}\right\}$ and for each $D_{j}$, in order, collapse $\gamma a D \searrow \gamma a \dot{D}$ by removing in pairs a horizontal simplex and the vertical simplex above it. This is clearly relative to $K$. The image of this collapse under $f$ is a collapse: $f(\gamma a K)=L^{k}, L^{k-1}, \ldots, L^{0}=f(a)$, with $L^{i+1}=L^{i}+A^{i}+B^{i}$ with $B^{i}=b^{i} A^{i}$ and $B^{i} \cap L^{i}$ $=b^{i} A^{i}$ and $f^{-1} A^{i}$ horizontal in $\gamma a K$ and $f^{-1} B^{i}$ the vertical simplex above it.

First we assume the following:

ASSERTION. Let $\left(V^{i}, V_{0}^{i},\left\{V_{j}^{i}\right\}\right)$ be a regular neighborhood of $L^{i} \bmod L^{i} \cap f(K)$ in $\left(J, f(a K),\left\{f\left(a D_{j}\right)\right\}\right)$. Then there exists a homeomorphism $h_{i}:\left(V^{i}, V_{0}^{i},\left\{V_{j}^{k}\right\}\right)$ $\rightarrow\left(V^{0}, V_{0}^{0},\left\{V_{j}^{0}\right\}\right)$, taking boundary to boundary.

All the work and in particular, the inductive application of Theorem 4, is contained in the proof of the assertion.

From the assertion, there exists

$$
h_{k}:\left(V, f(a K),\left\{f\left(a D_{j}\right)\right\}\right) \rightarrow \operatorname{St}\left(a ;\left(\eta J, \eta f(\gamma a K),\left\{\eta f\left(\gamma a D_{j}\right)\right\}\right)\right)
$$

taking $\left(\dot{V}, f(K),\left\{f\left(D_{j}\right)\right\}\right)$ to the corresponding link family. Thus we can define:

$$
g=\left(a *\left(h_{k} \mid \dot{V}\right)^{-1}\right) h_{k}:\left(V, f(a K),\left\{f\left(a D_{j}\right)\right\}\right) \rightarrow a *\left(\dot{V}, f(K),\left\{f\left(D_{j}\right)\right\}\right)
$$

rel $\dot{V}$. Now $g f \bar{f}^{-1}$ is a homeomorphism of $a * f(K)$ with itself rel $f(K)$ and mapping $a * f\left(D_{j}\right)$ to itself for all $j$. Since $\dot{V}$ can be triangulated with $f(K)$ as a subcomplex (e.g. map $\dot{V}$ to $\dot{N}\left(f(a K)-f(K) ; \eta_{f} J\right)$ rel $f(K)$ where $\eta_{f} J$ is a derived of $J \bmod f(\gamma a K)$ ), $a \dot{V}$ can be triangulated with $a f(K)$ as a subcomplex. The homeomorphism of $a f(K) \cup \dot{V}$ given by $g f \bar{f}^{-1}$ on $a f(K)$ and the identity on $\dot{V}$ maps each simplex of such a triangulation to itself and so extends to a homeomorphism of $a \dot{V}$ by induction up the dimension of the remaining simplices. Call this extension $\bar{g}: a(\dot{V}, f(K))$ $\rightarrow a(\dot{V}, f(K))$ rel $\dot{V}$. Let

$$
h=\bar{g}^{-1} g: V \rightarrow a \dot{V}
$$

rel $\dot{V}$, then $h f=\bar{f}$.

This reduces the proof of the theorem to a proof of the above assertion. Clearly, it suffices to prove the following:

AsSERTION'. There exists a homeomorphism $k_{i}$, for each $i$,

$$
k_{i}:\left(V^{i+1}, V_{0}^{i+1},\left\{V_{j}^{i+1}\right\}\right) \rightarrow\left(V^{i}, V_{0}^{i},\left\{V_{j}^{i}\right\}\right)
$$

and such that

$$
k_{i}\left(\dot{V}^{i+1}, \dot{V}_{0}^{i+1},\left\{\dot{V}_{j}^{i+1}\right\}\right)=\left(\dot{V}^{i}, \dot{V}_{0}^{i},\left\{\dot{V}_{j}^{i}\right\}\right)
$$


This assertion is seen to be just a "family" version of Theorem III.8. Thus, the proof will completely parallel that one, but with the difficulty, as will be seen, appearing at an unexpected point.

By the uniqueness theorem for regular neighborhoods, [C; Addendum 3.3] it suffices to construct such a $k_{i}$, for any choice of $V^{i+1}$ and any choice of $V^{i}$.

Now $L^{i+1}=L^{i}+A+B$ with $B=b A, L^{i} \cap B=b A$ and $f^{-1} B$ the vertical simplex above the horizontal simplex $f^{-1} A$. As in Theorem III.8, there are two cases: $A \in f K$ or $A \notin f K$.

Case (1) $A \in f K$. This corresponds to case (2) of the proof of Theorem III.8. There it is directly proved that there exist deriveds $\eta \sigma_{A} J$ and $\bar{\eta} \sigma_{A} J$ agreeing outside $\mathrm{St}^{\circ}(B, J)$ and a homeomorphism:

$$
k: N\left(L^{i+1} \cup f(K)-f(K) ; \eta \sigma_{A}(J, f(\gamma a K))\right) \rightarrow N\left(L^{i} \cup f(K)-f(K) ; \bar{\eta} \sigma_{A}(J, f(\gamma a K))\right)
$$

taking boundary pairs to boundary pairs. The naturality of the construction is such that if $D_{j} \in \mathrm{St}^{\circ}\left(f^{-1} A ; K\right)$ then

$$
k\left(N\left(L^{i+1} \cup f K-f K ; \eta \sigma_{A} f\left(\gamma a D_{j}\right)\right)\right)=N\left(L^{i} \cup f K-f K ; \bar{\eta} \sigma_{A} f\left(\gamma a D_{j}\right)\right)
$$

and similarly for the boundaries. For in $\mathrm{St}^{\circ}\left(B ; f\left(\gamma a D_{j}\right)\right)$ where the two neighborhoods differ, both are parametrized by the same $p: \operatorname{St}(B) \rightarrow \Delta^{3}$ as before.

Finally, if $D_{j} \in K-\mathrm{St}^{\circ}\left(f^{-1} A ; K\right)$, then $\eta$ and $\bar{\eta}$ are the same on $f\left(\gamma a D_{j}\right)$, $L^{i+1} \cap f\left(a D_{j}\right)=L^{i} \cap f\left(a D_{j}\right)$ and $k$ on its domain intersect $f\left(a D_{j}\right)$ is the identity.

Thus, if we let $\left(V^{i+1}, V_{0}^{i+1},\left\{V_{j}^{i+1}\right\}\right)=N\left(L^{i+1} \cup f K-f K ; \eta \sigma_{A}\left(J, f(\gamma a K),\left\{f\left(\gamma a D_{j}\right)\right\}\right)\right)$ and $\left(V^{i}, V_{0}^{i},\left\{V_{j}^{i}\right\}\right)=N\left(L^{i} \cup f K-f K ; \bar{\eta} \sigma_{A}\left(J, f(\gamma a K),\left\{f\left(\gamma a D_{j}\right)\right\}\right)\right)$, then the $k$ constructed as above following the proof of Theorem III.8 is the required $k_{i}$.

Case (2) $A \notin f K$. This corresponds to case (1) of Theorem III.8, or more precisely to case (1) of the proof of Theorem III.6. There it is shown directly, that there exist deriveds $\eta \sigma_{A} J$ and $\bar{\eta} \alpha J$ (where $\alpha J=\sigma_{b a} \sigma_{A} J$ ) agreeing outside of $\operatorname{St}^{\circ}(B ; J$ ) and such that:

$$
\left|N\left(L^{i}-L^{i} \cap f K ; \eta \sigma_{A}(J, f(\gamma a K))\right)\right|=\left|N\left(\left(L^{i}+x b \not{A}\right)-L^{i} \cap f K ; \bar{\eta} \alpha(J, f(\gamma a K))\right)\right| .
$$

As before, naturality of the construction, will imply that for $D_{j} \in \mathrm{St}^{\circ}\left(\pi f^{-1} A ; K\right)$,

$$
\left|N\left(L^{i}-L^{i} \cap f K ; \eta \sigma_{A} f\left(\gamma a D_{j}\right)\right)\right|=\left|N\left(\left(L^{i}+x b A\right)-L^{i} \cap f K ; \bar{\eta} \alpha f\left(\gamma a D_{j}\right)\right)\right| .
$$

For $D_{j} \in K-\mathrm{St}^{\circ}\left(\pi f^{-1} A ; K\right)$ this is clear because $L^{i} \cap f\left(a D_{j}\right)=\left(L^{i}+x b A\right)$ $\cap f\left(a D_{j}\right)$ and the two subdivisions of $f\left(\gamma a D_{j}\right)$ are the same, as $f\left(\gamma a D_{j}\right) \cap \mathrm{St}^{\circ}(B, J)$ $=\varnothing$ in this case.

Thus, if we let $\left(V^{i}, V_{0}^{i},\left\{V_{j}^{i}\right\}\right)=N\left(L^{i}-L^{i} \cap f K ; \eta \sigma_{A}\left(J, f(\gamma a K),\left\{f\left(\gamma a D_{j}\right)\right\}\right)\right)$.

Then, this is also a regular neighborhood of $L^{i}+x b A \bmod L^{i} \cap f K$ in $(J, f(a K)$, $\left.\left\{f\left(a D_{j}\right)\right\}\right)$.

From this stage to the end of the proof of Theorem III.6 is a simple little application of Corollary II.15 to push $x b \dot{A}$ out to $B$ rel $L^{i}$. This is, of course, the rub because if we just apply Corollary II.15 as before the resulting homeomorphism 
may mess up the cone structure i.e. it may not map the $f\left(a D_{j}\right)$ into themselves. What we need is the following strengthening of Corollary II.15 to this situation:

MAP. There exists a homeomorphism

$$
m:\left(J, f(a K),\left\{f\left(a D_{j}\right)\right\}\right) \rightarrow\left(J, f(a K),\left\{f\left(a D_{j}\right)\right\}\right)
$$

such that $m \mid L^{i}=$ identity and $m(B)=x b A$.

Assuming the existence of $m$, define $\left(V^{i+1}, V_{0}^{i+1},\left\{V_{0}^{i+1}\right\}\right)=m^{-1}\left(V^{i}, V_{0}^{i},\left\{V_{j}^{i}\right\}\right)$ and let $k^{i}=m \mid V^{i+1}$. Since $k^{i}$ is the restriction of a homeomorphism of the ambient spaces, it takes boundary into boundary.

Instead of proving Map, we prove the following sharpening of Lemma II.14b from which Map follows using the proof of Corollary II.15:

MAP'. Let $\eta_{B} J$ be a first derived $\bmod B$, then there exists a homeomorphism

$$
\phi: \operatorname{Lk}\left(A ; \eta_{B}(J, f(\gamma a K))\right) \rightarrow(b+x) \operatorname{Lk}\left(B ; \eta_{B}(J, f(\gamma a K))\right)
$$

such that

1. $\phi \mid b * \mathrm{Lk}\left(B ; \eta_{B} J\right)=$ identity.

2. For $D \in \mathrm{St}^{\circ}\left(\pi f^{-1} A ; K\right)$

$$
\phi\left(\operatorname{Lk}\left(A ; \eta_{B} f(\gamma a D)\right)\right)=(b+x) \operatorname{Lk}\left(B ; \eta_{B} f(\gamma a D)\right) .
$$

Condition 2 is precisely what is required to insure that if $m$ is defined using $\phi$ as in the proof of Corollary II.15, $m\left(f\left(a D_{j}\right)\right)=f\left(a D_{j}\right)$ for all $D_{j} \in K$. We only have to worry about $D \in \mathrm{St}^{\circ}\left(\pi f^{-1} A ; K\right)$ because $m$ will be the identity on the other $f\left(a D_{j}\right)$ 's.

We prove Map' in a sequence of claims:

Claim A. There exists a homeomorphism

$$
\phi_{0}: \operatorname{Lk}\left(A ; \eta_{B}(J, f(\gamma a K))\right) \rightarrow(b+x) \operatorname{Lk}\left(B ; \eta_{B}(J, f(\gamma a K))\right)
$$

with $\phi_{0} \mid b * \operatorname{Lk}\left(B ; \eta_{B} J\right)=$ identity.

Proof. By the assumption of conewise homogeneity, Lemma 3 implies that $A$ is a nice face of $B$ in $(J, f(\gamma a K))$. So we can apply Lemma II.14b.

Notation. For convenience, we will use $A, B$ and $b$ to denote $f^{-1} A, f^{-1} B$ and $f^{-1} b$, also, and allow context to determine whether it lies in the domain or range. Also, we let $\eta_{B} \gamma a K$ be the derived $\bmod B$ (i.e. $f^{-1} B$ ) of $\gamma a K$, such that $f: \eta_{B} \gamma a K$ $\rightarrow \eta_{B} f(\gamma a K)$ is simplicial.

Claim B. There exists a homeomorphism

$$
\phi: \operatorname{Lk}\left(A ; \eta_{B} \gamma a K\right) \rightarrow(b+x) \operatorname{Lk}\left(B ; \eta_{B} \gamma a K\right)
$$

such that:

1. $\phi \mid b * \mathrm{Lk}\left(B ; \eta_{B} \gamma a K\right)=$ identity.

2. $\phi\left(\operatorname{Lk}\left(A ; \eta_{B} \gamma a D\right)\right)=(b+x) \operatorname{Lk}\left(B ; \eta_{B} \gamma a D\right)$ for $D \in \mathrm{St}^{\circ}(\pi A ; K)$. 
Proof. Let $C=\pi A$. Then since $A \in \operatorname{Int} a C$ and $\operatorname{dim} A=\operatorname{dim} C=\operatorname{dim} a C-1$, Lk $\left(A ; \eta_{B} \gamma a C\right)$ is two points. One of them is $b$ and call the other $x$. Then define $\phi_{-1}(b+x)=b+x$ and this defines

$$
\phi_{-1}: \operatorname{Lk}\left(A ; \eta_{B} \gamma a C\right) \rightarrow(b+x) \operatorname{Lk}\left(B ; \eta_{B} \gamma a C\right) .
$$

Let $S^{i}=C * \operatorname{Lk}(C ; K)^{(i)}$ and $S^{-1}=C$. We define inductively

$$
\phi_{i}: \operatorname{Lk}\left(A ; \eta_{B} \gamma a S^{i}\right) \rightarrow(b+x) \operatorname{Lk}\left(B ; \eta_{B} \gamma a S^{i}\right)
$$

with $\phi_{i}$ the identity on $b * \operatorname{Lk}\left(B ; \eta_{B} \gamma a S^{i}\right)$ and satisfying 2 for any $D \in \operatorname{St}^{\circ}\left(C ; S^{i}\right)$, i.e. for any $D$ of dimension $\leqq \operatorname{dim} C+i+1$.

As we have defined $\phi_{-1}$ above, we will assume that $\phi_{i-1}$ is defined and construct $\phi_{i}$ to extend it.

Let $D \in S^{i}-S^{i-1}$, i.e. $D=C E$ with $\operatorname{dim} E=i$. We want to define

$$
\phi_{i}: \operatorname{Lk}\left(A ; \eta_{B} \gamma a D\right) \rightarrow(b+x) \operatorname{Lk}\left(B ; \eta_{B} \gamma a D\right)
$$

to extend the identity on $b \operatorname{Lk}\left(B ; \eta_{B} \gamma a D\right)$ and $\bar{\phi}_{i-1}$ on $\operatorname{Lk}\left(A ; \eta_{B} \gamma a * C \dot{E}\right)$. Doing this for each $E \in \operatorname{Lk}(C, K)^{(i)}-\mathrm{Lk}(C ; K)^{(i-1)}$ defines the required $\phi_{i}$.

Let $j=\operatorname{dim} A=\operatorname{dim} C$, then $\eta_{B} \gamma a C *(E, E)$ is an $i+j+2$ ball-and-face pair. $A$ and $B$ are interior $j$ and $j+1$ simplices of $\eta_{B} \gamma a C \dot{E}$. So $\operatorname{Lk}\left(A ; \eta_{B} \gamma a C *(E, E)\right)$ and $(b+x) \mathrm{Lk}\left(B ; \eta_{B} \gamma a C(E, \dot{E})\right)$ are $i+1$ ball-and-boundary pairs.

On the other hand, $b \mathrm{Lk}\left(B ; \eta_{B} \gamma a C E\right)$ is an $i+1$ ball contained in each of these and meeting each boundary in $b \mathrm{Lk}\left(B ; \eta_{B} \gamma a C \dot{E}\right)$, a face.

Hence, by the Alexander-Newman theorem, we can define $\phi_{i}$ on Lk $\left(A ; \eta_{B} \gamma a C E\right)$ to extend $\phi_{i-1}+$ identity on $\mathrm{Lk}\left(A ; \eta_{B} \gamma a C \dot{E}\right)+b \mathrm{Lk}\left(B ; \eta_{B} \gamma a C E\right)$.

This defines $\phi_{i}$ by induction and we define $\phi=\phi_{i}$ for $i=\operatorname{dim~Lk}(C ; K)$.

Claim C. $\phi_{0} f \bar{\phi}^{-1} \mid x \mathrm{Lk}\left(B ; \eta_{B} \gamma a K\right)$ is $\mathrm{CH}$, where

$$
\phi_{0} f \phi^{-1}: x \operatorname{Lk}\left(B ; \eta_{B} \gamma a K\right) \rightarrow(b+x) \operatorname{Lk}\left(B ; \eta_{B} J\right) .
$$

Proof. Since the definition of conewise homogeneity is an invariant under homeomorphism of the range, it suffices to show that

$$
f \bar{\phi}^{-1}: x \operatorname{Lk}\left(B ; \eta_{B} \gamma a K\right) \rightarrow \operatorname{Lk}\left(A ; \eta_{B} J\right)
$$

is $\mathrm{CH}$. If $y \in x \mathrm{Lk}\left(B ; \eta_{B} \gamma a K\right)$ then

$$
\begin{aligned}
d\left(f \bar{\phi}^{-1}(y) ; \operatorname{Lk}\left(A ; \eta_{B} J\right), f \bar{\phi}^{-1} x \operatorname{Lk}\left(B ; \eta_{B} \gamma a K\right)\right) & =d\left(f \bar{\phi}^{-1}(y) ; \operatorname{Lk}\left(A ; \eta_{B}(J ; \gamma a K)\right)\right) \\
& =d\left(f \bar{\phi}^{-1}(y) ; J, f(a K)\right)-\operatorname{dim} A-1
\end{aligned}
$$

with the last equality from Lemma II.19.

Thus, it suffices to show that $d\left(f \bar{\phi}^{-1}(y) ; J, f(a K)\right)$ is constant as $y$ varies along a cone-line: $\left\{t x+(1-t) y_{1}: 0<t<1\right\}$ with $y_{1} \in \operatorname{Lk}\left(B ; \eta_{B} \gamma a K\right)$.

There is a unique $D \in \mathrm{St}^{\circ}(C ; K)$ with $y_{1} \in \operatorname{Int} a D$. Let $D=C E$ and $y=t x+$ $(1-t) y_{1}$ with $0<t<1$. Then by condition 2 on $\phi, \phi^{-1}(y) \in \operatorname{Lk}\left(A ; \eta_{B} \gamma a C E\right)$ $-\operatorname{Lk}\left(A ; \eta_{B} \gamma a C \dot{E}\right)$. From this it follows that $\phi^{-1}(y) \in a D-a \dot{D}=a C E-a C \dot{E}-a \dot{C} E$, 
and since $A$ is an interior simplex, $\operatorname{Lk}\left(A ; \eta_{B} \gamma a K\right) \cap K$ contains at most $b$ and so $\phi^{-1}(y) \in$ Int $a D$.

Thus, for all $0<t<1, \phi^{-1}\left(t x+(1-t) y_{1}\right) \in$ Int $a D$, so the result follows from Lemma 3.

MAP'. The required $\phi$ exists.

Proof. $\phi_{0} f \phi^{-1}$ on $b * \operatorname{Lk}\left(B ; \eta_{B} \gamma a K\right)$ is just $b * f \mid \operatorname{Lk}(B)$ and hence has the image $b * \operatorname{Lk}\left(B ; \eta_{B} f(\gamma a K)\right)$. So $\phi_{0} f \phi^{-1}$ maps the pair $\left(x * \operatorname{Lk}\left(B ; \eta_{B} \gamma a K\right), \operatorname{Lk}\left(B ; \eta_{B} \gamma a K\right)\right)$ homeomorphically onto $\left(x * \operatorname{Lk}\left(B ; \eta_{B} f \gamma a K\right), \operatorname{Lk}\left(B ; \eta_{B} f \gamma a K\right)\right)$, and by $C$, it is $\mathrm{CH}$ regarded as an embedding into $(b+x) \mathrm{Lk}\left(B ; \eta_{B} J\right)$.

Clearly, $x * \operatorname{Lk}\left(B ; \eta_{B} J\right)$ is a regular neighborhood of

$$
x \operatorname{Lk}\left(B ; \eta_{B} f \gamma a K\right) \bmod \operatorname{Lk}\left(B ; \eta_{B} f \gamma a K\right)
$$

in $(b+x) \mathrm{Lk}\left(B ; \eta_{B} J\right)$, with boundary $\mathrm{Lk}\left(B ; \eta_{B} J\right)$.

By an inductive application of Theorem 4 , there exists a homeomorphism

$$
h: x * \operatorname{Lk}\left(B ; \eta_{B} J\right) \rightarrow x * \operatorname{Lk}\left(B ; \eta_{B} J\right)
$$

rel Lk $\left(B ; \eta_{B} J\right)$ such that, $h \phi_{0} f \phi^{-1}=x *\left(f \mid \operatorname{Lk}\left(B ; \eta_{B} \gamma a K\right)\right)$.

Extend $h$ over $b * \operatorname{Lk}\left(B ; \eta_{B} J\right)$ by the identity and call the extension $h_{1}$.

Define $\phi=h_{1} \phi_{0}$.

Clearly, $\phi \mid b * \operatorname{Lk}\left(B ; \eta_{B} J\right)$ is the identity

$$
h\left(x * \operatorname{Lk}\left(B ; \eta_{B} f \gamma a K\right)\right)=x * \operatorname{Lk}\left(B ; \eta_{B} f \gamma a K\right)
$$

and so $\phi\left(\operatorname{Lk}\left(A ; \eta_{B} f \gamma a K\right)\right)=(b+x) \operatorname{Lk}\left(B ; \eta_{B} f \gamma a K\right)$.

Finally, $\phi \cdot(f \mid \operatorname{Lk}(A))=((b+x) *(f \mid \operatorname{Lk}(B))) \cdot \phi$ and hence for $D \in \operatorname{St}^{\circ}(C ; K)$ :

$$
\begin{aligned}
\phi\left(\operatorname{Lk}\left(A ; \eta_{B} f \gamma a D\right)\right)=\phi f\left(\operatorname{Lk}\left(A ; \eta_{B} \gamma a D\right)\right) & =((b+x) * f)(b+x) * \operatorname{Lk}\left(B ; \eta_{B} \gamma a D\right) \\
& =(b+x) * \operatorname{Lk}\left(B ; \eta_{B} f \gamma a D\right) .
\end{aligned}
$$

Thus, $\phi$ is the required homeomorphism.

This completes the proof of Map' and hence the theorem.

From Theorem 4, we obtain several corollaries about unknotting cone pairs.

Call an embedding $f: a X \rightarrow b Y$ a proper embedding if $f^{-1}(Y)=X$.

DEFINITION 5. We call a proper embedding $f: a X \rightarrow b Y$ unknotted if there exists a homeomorphism $h: b Y \rightarrow b Y$ rel $Y$ such that $h f=\bar{f}=$ cone $f \mid X$.

COROLlaRY 6. Let $f: a X \rightarrow b Y$ be a proper embedding, then $f$ is unknotted iff

1. $f$ is conewise homogenous, and

2. $(b Y \mid Y)_{w} \searrow f(a X)$ in $b Y$.

Proof. Lemma 2 implies that 1 is necessary and if $f$ is unknotted then the pair $(b Y, f(a X)) \cong b *(Y, f(X))$ and the conewise collapse of $b Y$ to $b f(X)$ is rel $Y$ and weakly homogenous in $b Y$ and induces under the homeomorphism a similar collapse to $f(a X)$. 
Since $b Y$ is a regular neighborhood of $b Y \bmod Y$ in $b Y$, Theorem III.8 implies that there exists a homeomorphism

$$
k:(b Y, Y) \rightarrow(V, \dot{V})
$$

rel $f(a X)$, where $V$ is a regular neighborhood of $f(a X) \bmod f(X)$ in $b Y$.

By Theorem 4 , there is a homeomorphism $h_{1}: V \rightarrow b \dot{V}$ rel $\dot{V}$ such that $h_{1} f=\bar{f}$.

Let $h=\left(b *(k \mid Y)^{-1}\right) h_{1} k$.

COROLlaRY 7. Let $f: a X \rightarrow b Y$ be a proper embedding with $(b Y, f(a X))$ locally unknotted at every point of $f(a X)-f(X)$. Then $f$ is unknotted iff $\left(b Y|Y|{ }_{w} \searrow f(a X)\right.$ in $b Y$.

Proof. Since the local unknottedness condition implies $f$ is $\mathrm{CH}$, this is clear from Corollary 6.

COROllaRY 8. Let $a\left(X, X_{0}\right)$ be $a$ cone pair and $h: a X_{0} \rightarrow a X_{0}$ a homeomorphism rel $X_{0}$. Then $h$ can be covered by a homeomorphism $H: a X \rightarrow a X$ rel $X$, iff regarded as an embedding of $a X_{0} \rightarrow a X, h$ is conewise homogenous.

Proof. $\bar{h}=$ inclusion $a X_{0} \subset a X$ so $H: a X \rightarrow a X$ rel $X$ extends $h$ iff $H^{-1}$ unknots the embedding $h: a X_{0} \rightarrow a X$. Since $(a X \mid X)_{w} \searrow a X_{0}$ in $a X, h$ unknots iff it is conewise homogenous by Corollary 6.

REMARKS. If $f_{0}=f_{1}: a X \rightarrow b Y$ are proper unknotted embeddings with $f_{0}\left|X=f_{1}\right| X$ then $f_{0}$ is ambient isotopic to $f_{1}$ rel $Y$. For if $h_{0}, h_{1}: b Y \rightarrow b Y$ rel $Y$ unknot the two embeddings, then $h_{0} h_{1}^{-1}$ is ambient isotopic to the identity rel $Y$.

Note that if $f: c X_{0} \cong c X_{0}$ rel $X_{0} \cup c\left(X_{0} \cap Y\right)$, where $X_{0}, Y<X$, is conewise homogenous, regarded as an embedding of $c X_{0}$ into $c X$, then so is $f \cup$ identity: $c\left(X_{0} \cup Y\right)=c X_{0} \cup c Y \rightarrow c X_{0} \cup c Y$, which gives a relative form of Corollary 8 .

V. Codimension three unknotting criteria. In this section we apply the sunny collapsing lemma of Stallings-Zeeman-Lickorish-Hudson to obtain much stronger theorems on unknotting cones in what may be called the codimension $\geqq 3$ situation.

Definition 1. A pair $\left(X, X_{0}\right)$ is called a codimension $\geqq 3$ pair if it satisfies the condition, for all $i$

$$
\operatorname{dim} X_{0} \cap I^{i}(X) \leqq i-3 .
$$

This notion is singled out because we will repeatedly use the fact that it is inductive, i.e.

Lemma 2. Let $\left(K, K_{0}\right)$ be a codimension $\geqq 3$ pair of complexes and $A \in K_{0}$. Then the pair Lk $\left(A ; K, K_{0}\right)$ is a codimension $\geqq 3$ pair.

Proof. Let $\operatorname{dim} A=j$. By Corollary II.18

$$
\begin{aligned}
\operatorname{Lk}\left(A ; K_{0}\right) \cap I^{i}(\operatorname{Lk}(A ; K)) & =\operatorname{Lk}\left(A ; K_{0}\right) \cap \operatorname{Lk}\left(A ; I^{i+j+1}(K)\right) \\
& =\operatorname{Lk}\left(A ; K_{0} \cap I^{i+j+1}(K)\right) . \\
\operatorname{dim} K_{0} \cap I^{i+j+1}(K) & \leqq(i+j+1)-3 .
\end{aligned}
$$


So $\operatorname{dim} \operatorname{Lk}\left(A ; K_{0} \cap I^{i+j+1}(K)\right) \leqq(i+j+1-3)-(j+1)=i-3$.

We will also need the following technical remark, which we will dignify as a lemma because we use it repeatedly:

Lemma 3. Consider a pair $\left(X, X_{0}\right)$ and an embedding $g:\left(v_{0}+v_{1}\right) X_{0} \rightarrow\left(v_{0}+v_{1}\right) X$ such that

1. $g \mid v_{0} * X_{0}$ is the inclusion.

2. $g \mid v_{1} * X_{0}$ maps $v_{1} * X_{0}$ into $v_{1} * X$ and is a proper unknotted embedding of cones.

Then there exists $G:\left(v_{0}+v_{1}\right) X \rightarrow\left(v_{0}+v_{1}\right) X$ rel $v_{0} * X$ such that $G g=$ inclusion.

Proof. If $H: v_{1} * X \rightarrow v_{1} * X$ unknots $g \mid v_{1} * X_{0}$ then let $G=H$ on $v_{1} * X$ and identify on $v_{0} * X$.

Now we state the main theorem of this section:

THEOREM 4. Let $\left(X, X_{0}\right)$ be a compact codimension $\geqq 3$ pair and $f: a X_{0} \rightarrow a X$ a proper embedding with $f \mid X_{0}=$ inclusion. Assume that for all $i, f^{-1}\left(a I^{i}(X)\right)=$ $a *\left(X_{0} \cap I^{i}(X)\right)$ and $f(a) \in a * S(X)$. Then the embedding is unknotted.

REMARKS. The fact that $X_{0}<X$ is a merely formal restriction for convenience. To apply this theorem to a proper embedding $f: a X_{0} \rightarrow a X$, just identify $X_{0}$ with $f X_{0}$ by $f$ and $f$ with $f \cdot a *\left(f \mid X_{0}\right)^{-1}$.

The assumption $f(a) \in a * S(X)$ can be included in the first assumption if we recall our convention that $I^{-1}(X)=\{0\}$ if $X$ is not a suspension and assume that $a *\left(X_{0} \cap I^{i}(X)\right)=a$ if $X_{0}$ and $I^{i}(X)$ are disjoint.

We begin by examining the condition put on the embedding:

LemmA 5. Let $f: a X_{0} \rightarrow a X$ be a proper embedding. Then the following are equivalent:

a. $f^{-1}\left(a * I^{i}(X)\right)=a *\left(f \mid X_{0}\right)^{-1}\left(I^{i}(X)\right)$ and $f(a) \in a * S(X)$.

b. For all $x \in a X_{0}-X_{0}, d(\bar{f}(x) ; a X)=d(f(x) ; a X)$.

c. For all $x \in a X_{0}-X_{0}, \bar{f}(x) \sim f(x)$ in $a X$.

d. $d(a ; a X)=d(f(a) ; a X)$ and if $x=t a+(1-t) x_{1}$, with $0<t<1$ and $x_{1} \in X_{0}$, then $d(f(x) ; a X)=d\left(f\left(x_{1}\right) ; X\right)+1$.

Note that if $f \mid X_{0}$ is an inclusion, then $\bar{f}$ is an inclusion.

Proof. $\mathrm{a} \rightarrow \mathrm{c}$. If $x=a$, this is clear. If $x \in a X_{0}-\left(X_{0} \cup a\right)$ then $x=t a+(1-t) x_{1}$ for $x_{1} \in X_{0}$ and $0<t<1$. Let $\overline{x x}_{1}$ be the path along this cone line from $x$ to $x_{1}$. If $x \in a *\left(f \mid X_{0}\right)^{-1}\left(I^{i}(X)-I^{i-1}(X)\right)$, then $f\left(\overline{x x}_{1}\right)+\bar{f}\left(\overline{x x}_{1}^{-1}\right)$ (addition and inverse of paths) is a path joining $f(x)$ to $\bar{f}(x)$ in $a I^{i}(X)-a I^{i-1}(X)$ which is an $i$ manifold with boundary $I^{i}-I^{i-1}$. The path meets the boundary at one point $f\left(x_{1}\right)$ and so can be pushed off the boundary rel the end points by local collaring.

$\mathrm{c} \rightarrow$ b. Clear.

$\mathrm{b} \rightarrow \mathrm{a} . \bar{f}^{-1}\left(a * I^{i}\right)=a *\left(f \mid X_{0}\right)^{-1}\left(I^{i}\right)$ and $\mathrm{b}$ implies the left side equals $f^{-1}\left(a * I^{i}\right)$. Explicitly at the vertex we have $d(f(a) ; X)=d(a ; X)=\operatorname{dim} S(X)+1$. Points of 
$a X-(a S(X) \cup X)$ have higher intrinsic dimension and so since $f(a) \notin X$ ( $f$ is a proper embedding) $f(a) \in a S(X)$.

$\mathrm{b} \rightleftarrows \mathrm{d} . d(\bar{f}(x) ; a X)=d\left(f\left(x_{1}\right) ; X\right)+1$.

Now we begin the proof of Theorem 4 . There are essentially two parts to the proof. There is a collapsing statement proved using the sunny collapsing lemma, and then an inductive argument.

Lemma 6. Let $\left(X, X_{0}\right)$ and $f: a X_{0} \rightarrow a X$ satisfy the hypothesis of Theorem 4 and in addition, assume that $\left(X, X_{0}\right) \subset\left(a X, f\left(a X_{0}\right)\right)$ is locally collared. Then $(a X \mid X)$ ${ }_{w} \searrow f\left(a X_{0}\right)$ in $a X$.

Proof. The local collaring assumption implies that there is a homeomorphism

$$
h:\left(a X, f\left(a X_{0}\right)\right) \rightarrow\left(a X, f\left(a X_{0}\right)\right) \cup_{\left(X, X_{0}\right)}\left(X \times I, X_{0} \times I\right)
$$

such that $h(x)=(x, 1)$ for $x \in X$ (see Theorem IV.2).

So it suffices to show that

$$
(a X \cup X \times I \mid X \times 1){ }_{w} \searrow f\left(a X_{0}\right) \cup X_{0} \times I \text { in } a X \cup X \times I .
$$

We begin by the obvious cylindrical collapse:

$$
(a X \cup X \times I \mid X \times 1)_{w} \searrow a X \cup X_{0} \times I \text { in } a X \cup X \times I
$$

given by $(X \times I \mid X \times 1) \searrow X \times 0 \cup X_{0} \times I$ in $X \times I$.

So now, by excision, it suffices to show that $a X_{n} \searrow f\left(a X_{0}\right)$ in $a X \cup X \times I$. So we will prove

$$
a I^{i}(X) \searrow f\left(a *\left(X_{0} \cap I^{i}(X)\right)\right) \cup a I^{i-1}(X) .
$$

Since $a I^{i}(X)=a X \cap I^{i+1}(a X \cup X \times I)$ and $f\left(a X_{0}\right) \cap a I^{i}(X)=f\left(a\left(X_{0} \cap I^{i}(X)\right)\right)$ by hypothesis, Lemma III.10 will then imply that $a X_{h} \searrow f\left(a X_{0}\right)$ in $a X \cup X \times I$.

To prove $a I^{i} \searrow f\left(a\left(X_{0} \cap I^{i}\right)\right) \cup a I^{i-1}$, we will require two sublemmas:

Sublemma A (SunNy Collapsing). Let $X_{0}<X<M$ with $M$ a compact manifold. Assume $M \searrow X_{0}, X \searrow X_{0}$ and $\operatorname{dim} M-\operatorname{dim} X-X_{0} \geqq 3$. Then $M \searrow X$.

Proof. See [H; Theorem 5.2].

Sublemma B (Marshall Cohen). Let $X \searrow X_{0}$ and $V_{0}$ be a regular neighborhood of $X_{0}$ in $X$. Then $X \searrow V_{0}$ and $X$-Int $V_{0} \searrow \dot{V}_{0}$.

Proof. By uniqueness of regular neighborhoods it suffices to prove it for any $V_{0}$. So triangulate with $K_{0}$ full to obtain $K \searrow^{s} K_{0}$ and let $V_{0}=N\left(K_{0} ; \eta K\right)$. It suffices to prove $C\left(K_{0} ; \eta K\right) \searrow \dot{N}\left(K_{0} ; \eta K\right)$.

If the simplicial collapse $K \searrow^{s} K_{0}$ is given by removing pairs of simplices: $\left(A_{n}, B_{n}\right), \ldots,\left(A_{0}, B_{0}\right)$ with $A_{i}<B_{i}$, then a geometrical collapse $C\left(K_{0} ; \eta K\right) \searrow$ $\dot{N}\left(K_{0} ; \eta K\right)$ is given by removing ball pairs: $\left(\bar{A}_{n}, \bar{B}_{n}\right), \ldots,\left(\bar{A}_{0}, \bar{B}_{0}\right)$ defined by $\bar{A}_{i}=C\left(A_{i} \cap K_{0} ; \eta A_{i}\right)$ and $\bar{B}_{i}=C\left(B_{i} \cap K_{0} ; \eta B_{i}\right)$. 
To prove $a I^{i} \searrow f\left(a\left(X_{0} \cap I^{i}\right)\right) \cup a I^{i-1}$ : By collapsing conewise $a I^{i} \searrow a I^{i-1}$, or in the lowest dimensional case $a S(X) \searrow f(a)$.

Also, by hypothesis $f\left(a\left(X_{0} \cap I^{i}\right)\right) \cap a I^{i-1}=f\left(a\left(X_{0} \cap I^{i-1}\right)\right)$. Hence, by excision, $f\left(a\left(X_{0} \cap I^{i}\right)\right) \cup a I^{i-1} \searrow a I^{i-1}$, or in the lowest dimensional case, $f\left(a\left(X_{0} \cap S(X)\right)\right)$ $\searrow f(a)$. Let $Q=a I^{i}$ or $a S(X), F=f\left(a\left(X_{0} \cap I^{i}\right)\right) \cup a I^{i-1}$ or $f\left(a\left(X_{0} \cap S(X)\right)\right)$ and $F_{0}=a I^{i-1}$, or $f(a)$. Then $Q \searrow F_{0}$ and $F \searrow F_{0}$ and we wish to show that $Q \searrow F$. Note that $\left(Q, F_{0}\right)$ is a relative manifold and the codimension $\geqq 3$ assumption states precisely that $F-F_{0}$ has codimension at least 3 in $Q-F_{0}$.

Let $\left(V, V_{0}\right)$ be a regular neighborhood of $F_{0}$ in $(Q, F)$. Let $\bar{Q}=Q-$ Int $V$ and $\vec{F}=\left(F-\right.$ Int $\left.V_{0}\right) \cup \dot{V}$ and $\bar{F}_{0}=\dot{V}$. By Sublemma B, $\bar{Q} \searrow \bar{F}_{0}$ and by Sublemma B and excision $\bar{F} \searrow \bar{F}_{0}$. Now $\bar{Q}$ is a compact manifold and $\bar{F}-\bar{F}_{0} \subset F-F_{0}$ has codimension at least three so sunny collapsing implies that $\bar{Q} \searrow \bar{F}$. So by excision $Q \searrow F \cup V$.

Now by [C; Proposition 7.10], $V$ is a regular neighborhood of $V_{0} \bmod \dot{V}_{0}$ in $Q$ so $V \searrow V_{0}$ and hence by excision $F \cup V \searrow F$.

Thus, $Q \searrow F \cup V \searrow F$, as required, and we have for all $i$

$$
a I^{i} \searrow f\left(a\left(X_{0} \cap I^{i}\right)\right) \cup a I^{i-1} .
$$

Proof of Theorem 4. We assume inductively that the theorem is true for $\operatorname{dim} X<n$ and prove it for $\operatorname{dim} X=n$. The proof is in two parts: $\mathrm{CH}: f$ is conewise homogenous and LC: $\left(X, X_{0}\right) \subset\left(a X, f\left(a X_{0}\right)\right)$ is locally collared. Lemma 6 and Corollary IV.6 then imply that $f$ is unknotted.

Triangulate to obtain $f: \gamma a K_{0} \rightarrow \beta a K$ a conical simplicial embedding with $\beta K=K$. $f \mid K_{0}=$ inclusion of $K_{0}$ into $K$. Let $i: a K_{0} \rightarrow a K$ be inclusion $=\bar{f}$. Our assumptions are: $\left(K, K_{0}\right)$ is a codimension $\geqq 3$ situation and

$$
f^{-1}\left(a I^{i}(K)\right)=a\left(I^{i}(K) \cap K_{0}\right) .
$$

$\mathrm{CH}: f$ is conewise homogenous.

Proof. By Lemma IV.3 it suffices to consider $A$ and $B=b A$ such that $f^{-1} A$ is a horizontal simplex and $f^{-1} B$ is a vertical simplex above or below it, both in $\gamma a K_{0}-\left(a \cup K_{0}\right)$, and prove that $A$ is a nice face of $B$ in $\left(\beta a K, f\left(\gamma a K_{0}\right)\right)$. We shall do this by showing that the link pair of $A$ is a suspension of the link pair of $B$. This we shall do in a sequence of claims.

Notation. Let $\eta_{B} \gamma a K_{0}$ and $\eta_{B} \beta a K$ be deriveds $\bmod f^{-1} B$ and $B$ respectively such that $f: \eta_{B} \gamma a K_{0} \rightarrow \eta_{B} \beta a K$ is simplicial.

Also, let $C=\pi f^{-1} A=\pi f^{-1} B \in K_{0}$.

Claim A. There exists a homeomorphism

$$
\psi: \operatorname{Lk}\left(A ; \eta_{B} \beta a K\right) \rightarrow(b+x) \operatorname{Lk}\left(B ; \eta_{B} \beta a K\right) \operatorname{rel} b * \operatorname{Lk}\left(B ; \eta_{B} \beta a K\right) .
$$

Proof. By Lemma 5, $f(a) \sim i(a) \sim i(b) \sim f(b)$ in $a K$ where $a$ and $b$ are interior points of $f^{-1} A$ and $f^{-1} B$ respectively. Hence, $A$ is a nice face of $B$ in $a K$ and the claim comes from Lemma II.14b. 
Claim B. There exists a homeomorphism

$$
\phi: \operatorname{Lk}\left(A ; \eta_{B} f\left(\gamma a K_{0}\right)\right) \rightarrow(b+x) \operatorname{Lk}\left(B ; \eta_{B} f\left(\gamma a K_{0}\right)\right)
$$

rel $b * \mathrm{Lk}\left(B ; \eta_{B} f\left(\gamma a K_{0}\right)\right)$ and such that if $D \in \mathrm{St}^{\circ}\left(C ; K_{0}\right)$ then

$$
\phi\left(\operatorname{Lk}\left(A ; \eta_{B} f(\gamma a D)\right)\right)=(b+x) \operatorname{Lk}\left(B ; \eta_{B} f(\gamma a D)\right) .
$$

Proof. This is essentially Claim B of the proof of Theorem IV.4. If $\phi$ is defined as it is there then $\phi=((b+x) * f) \phi f^{-1}$.

Claim C. $\psi \phi^{-1} \mid x \operatorname{Lk}\left(B ; \eta_{B} f\left(\gamma a K_{0}\right)\right)$ satisfies the hypotheses of Theorem 4, regarded as embedding

$$
x \operatorname{Lk}\left(B ; \eta_{B} f\left(\gamma a K_{0}\right)\right) \rightarrow x \operatorname{Lk}\left(B ; \eta_{B} \beta a K\right) .
$$

Proof. First, we show that $\operatorname{Lk}\left(B ; \eta_{B} \beta a K, \eta_{B} f\left(\gamma a K_{0}\right)\right)$ is a codimension $\geqq 3$ pair. As in Lemma 2, we have

$\operatorname{Lk}\left(B ; \eta_{B} f\left(\gamma a K_{0}\right)\right) \cap I^{i}\left(\operatorname{Lk}\left(B ; \eta_{B} \beta a K\right)\right)=\operatorname{Lk}\left(B ; \eta_{B} f\left(\gamma a K_{0}\right) \cap I^{i+j+1}\left(\eta_{B} \beta a K\right)\right)$.

Since $B \notin K_{0}$, this equals, in turn

$$
\operatorname{Lk}\left(B ; \mathrm{Cl}\left(\eta_{B} f\left(\gamma a K_{0}\right) \cap I^{i+j+1}\left(\eta_{B} \beta a K\right)-K\right)\right) .
$$

So it suffices to know that $\operatorname{dim} f\left(a K_{0}\right) \cap I^{i+j+1}(a K)-K \leqq(i+j+1)-3$. But $f\left(a K_{0}\right) \cap I^{i+j+1}(a K)-K=f\left(a K_{0}\right) \cap a I^{i+j}(K)-K$ and by hypothesis on $f$ this is $f\left(a\left(K_{0} \cap I^{i+j}(K)\right)\right)$ which has dimension $\leqq i+j-2$ as required.

Next, if $y \in x \operatorname{Lk}\left(B ; \eta_{B} f\left(\gamma a K_{0}\right)\right)-\operatorname{Lk}\left(B ; \eta_{B} f\left(\gamma a K_{0}\right)\right)$ we invoke Lemma 5 and prove

$$
d\left(\psi \phi^{-1}(y) ; x \operatorname{Lk}\left(B ; \eta_{B} \beta a K\right)\right)=d\left(y ; x \operatorname{Lk}\left(B ; \eta_{B} \beta a K\right)\right) .
$$

These equal, respectively, the intrinsic dimension of $\psi \phi^{-1}(y)$ and $y$ in

$$
(b+x) \operatorname{Lk}\left(B ; \eta_{B} \beta a K\right) \text {. }
$$

If $y$ does not equal $x$, then $y=t x+(1-t) y_{1}$ with $0<t<1$ and

$$
y_{1} \in \operatorname{Lk}\left(B ; \eta_{B} f\left(\gamma a K_{0}\right)\right) \text {. }
$$

Clearly $y \sim y_{1}$ in $(b+x)$ Lk $\left(B ; \eta_{B} \beta a K\right)$.

There exists a unique $D \in \mathrm{St}^{\circ}\left(C ; K_{0}\right)$ with $y_{1} \in \operatorname{Int} \operatorname{Lk}\left(B ; \eta_{B} f(\gamma a D)\right)$, and $y_{1} \in \operatorname{Int} a D$. Since $y \in x \operatorname{Lk}\left(B ; \eta_{B} f(\gamma a D)\right)-x \operatorname{Lk}\left(B ; \eta_{B} f(\gamma a \dot{D})\right)$, it follows that $\phi^{-1}(y) \in \operatorname{Int} f(a D)$.

Hence, $d\left(y_{1} ; a K\right)=d\left(\phi^{-1}(y) ; a K\right)$ and hence by Lemma II.19,

$$
d\left(y_{1} ; \operatorname{Lk}\left(A ; \eta_{B} \beta a K\right)\right)=d\left(\phi^{-1}(y) ; \operatorname{Lk}\left(A ; \eta_{B} \beta a K\right)\right) .
$$

So

$$
d\left(\psi \phi^{-1}(y) ;(b+x) \operatorname{Lk}\left(B ; \eta_{B} \beta a K\right)\right)=d\left(y_{1} ;(b+x) \operatorname{Lk}\left(B ; \eta_{B} \beta a K\right)\right),
$$

which completes the proof when $y \neq x$ since $y_{1} \sim y$. 
As for $x, \phi^{-1}(x) \in \operatorname{Lk}\left(A ; \eta_{B} f(\gamma a C)\right)$ which consists of $b$ and this point. At any rate $A$ is a nice face of $A \phi^{-1}(x)$ in $\eta_{B} \beta a K$ since they are both interior to $\eta_{B} f(\gamma a C)$. Hence, $\phi^{-1}(x) \in S\left(\operatorname{Lk}\left(A ; \eta_{B} \beta a K\right)\right)$ and so

$$
\psi \phi^{-1}(x) \in S\left((b+x) \operatorname{Lk}\left(B ; \eta_{B} \beta a K\right)\right)=(b+x) S\left(\operatorname{Lk}\left(B, \eta_{B} \beta a K\right)\right) .
$$

So $\psi \phi^{-1}(x) \in x S\left(\operatorname{Lk}\left(B ; \eta_{B} \beta a K\right)\right)$.

Claim D. $\operatorname{Lk}\left(A ; \eta_{B}\left(\beta a K, f\left(\gamma a K_{0}\right)\right)\right) \cong \Sigma \operatorname{Lk}\left(B ; \eta_{B}\left(\beta a K, f\left(\gamma a K_{0}\right)\right)\right)$.

Proof. Consider the embedding:

$$
\psi \phi^{-1}:(b+x) \operatorname{Lk}\left(B ; \eta_{B} f\left(\gamma a K_{0}\right)\right) \rightarrow(b+x) \operatorname{Lk}\left(B ; \eta_{B} \beta a K\right) .
$$

By Claim $\mathrm{C}$ and an application of the inductive assumption on Theorem 4, this embedding satisfies the requirements of Lemma 3. Thus,

$$
\begin{aligned}
\left((b+x) \operatorname{Lk}\left(B ; \eta_{B} \beta a K\right), \psi \phi^{-1}(b+x) \operatorname{Lk}\right. & \left.\left(B ; \eta_{B} f\left(\gamma a K_{0}\right)\right)\right) \\
& \cong(b+x) \operatorname{Lk}\left(B ; \eta_{B} \beta a K, \eta_{B} f\left(\beta a K_{0}\right)\right),
\end{aligned}
$$

and the first pair is homeomorphic, by $\psi$ to

$$
\operatorname{Lk}\left(A ; \eta_{B} \beta a K, \eta_{B} f\left(\gamma a K_{0}\right)\right),
$$

which proves Claim D and hence completes the proof of $\mathrm{CH}$.

LC: $\left(K, K_{0}\right) \subset\left(a K, f\left(a K_{0}\right)\right)$ is locally collared.

Proof. Given a point $y$ of $K_{0}$ we have to show that $\operatorname{Lk}\left(y ; a K, f\left(a K_{0}\right)\right) \cong$ $c \mathrm{Lk}\left(y ; K, K_{0}\right)$. Clearly, by going to a subdivision if necessary, we can assume that $y=v$ is a vertex of $K_{0}$.

Notation. Let $\eta \gamma a K_{0}$ and $\eta \beta a K$ be deriveds such that $f: \eta \gamma a K_{0} \rightarrow \eta \beta a K$ is simplicial.

Claim A. There exists a homeomorphism

$$
\psi: \operatorname{Lk}(v ; \eta \beta a K) \rightarrow x \operatorname{Lk}(v ; \eta K) \operatorname{rel} \mathrm{Lk}(v ; \eta K) .
$$

Proof. This is because $K \subset a K$ is locally collared.

Claim B. There exists a homeomorphism

$$
\phi: \operatorname{Lk}\left(v ; \eta f\left(\gamma a K_{0}\right)\right) \rightarrow x \operatorname{Lk}\left(v ; \eta K_{0}\right) \operatorname{rel~Lk}\left(v ; \eta K_{0}\right)
$$

and such that if $D \in \mathrm{St}^{\circ}\left(v ; K_{0}\right)$ :

$$
\phi(\operatorname{Lk}(v ; \eta f(\gamma a D)))=x \operatorname{Lk}(v ; \eta D) .
$$

Proof. Pseudo-radial projection gives a homeomorphism

$$
\phi: \operatorname{Lk}\left(v ; \eta \gamma a K_{0}\right) \rightarrow \operatorname{Lk}\left(v ; a K_{0}\right)=a \operatorname{Lk}\left(v ; K_{0}\right)
$$

such that for $D \in \mathrm{St}^{\circ}\left(v ; K_{0}\right)$ :

$$
\phi(\operatorname{Lk}(v ; \eta \gamma a D))=\operatorname{Lk}(v ; a D) .
$$


So let $\phi=\left(x * f \mid \operatorname{Lk}\left(v ; \eta \gamma a K_{0}\right)\right)\left(x * \phi \mid \operatorname{Lk}\left(v ; K_{0}\right)\right)^{-1} \phi f^{-1}$.

Claim C. $\psi \phi^{-1}$ satisfies the hypotheses of Theorem 4 .

Proof. Lk $\left(v ; \eta K, \eta K_{0}\right)$ is a codimension $\geqq 3$ pair by Lemma 2 .

Now we check the intrinsic skeleton condition.

First, on the vertex $x . d\left(\psi \phi^{-1}(x) ; x \operatorname{Lk}(v ; \eta K)\right)=d\left(\phi^{-1}(x) ; \operatorname{Lk}(v ; \eta \beta a K)\right)$ $=d\left(\phi^{-1}(x) ; a K\right)-1$ by Corollary II.19. Now by Claim B, $\phi^{-1}(x)=\operatorname{Lk}(v ; \eta f(\gamma a v))$ and so $\phi^{-1}(x)=f(t a+(1-t) v)$ for some $0<t<1$. Hence, by Lemma $5 \mathrm{~d}$ applied to $f, d\left(\phi^{-1}(x) ; a K\right)=d(v ; K)+1$. Note that $d(v ; K)$ clearly equals $d(x ; x \operatorname{Lk}(v ; \eta K))$. So we have

$$
d\left(\psi \phi^{-1}(x) ; x \operatorname{Lk}(v ; \eta K)\right)=d(x ; x \operatorname{Lk}(v ; \eta K)) .
$$

Now let $y=t x+(1-t) y_{1}$ with $0<t<1$ and $y_{1} \in \operatorname{Lk}\left(v ; \eta K_{0}\right)$. We will show that

$$
d\left(\psi \phi^{-1}(y) ; x \operatorname{Lk}(v ; \eta K)\right)=d\left(y_{1} ; \operatorname{Lk}(v ; \eta K)\right)+1 .
$$

By Corollary II.19 again, it suffices to show

$$
d\left(\phi^{-1}(y) ; a K\right)=d\left(y_{1} ; K\right)+1 .
$$

However, if $y_{1} \in \operatorname{Int} \mathrm{Lk}(v ; \eta D)$ for $D \in \mathrm{St}^{\circ}\left(v ; K_{0}\right)$ then by Claim $\mathrm{B}, \phi^{-1}(y)$ $\in$ Int $f(a D)$. But by Lemma $5 \mathrm{~b} d\left(\phi^{-1}(y) ; a K\right)=d\left(f^{-1} \phi^{-1}(y) ; a K\right)$. Since $f^{-1} \phi^{-1}(y)$ $\in$ Int $a D$, clearly $d\left(f^{-1} \phi^{-1}(y) ; a K\right)=d\left(y_{1} ; K\right)+1$.

Thus, $\psi \phi^{-1}$ satisfies the hypotheses of Theorem 4 , by Lemma $5 \mathrm{~d}$.

Claim D. Lk $\left(v ; \eta \beta a K, \eta f\left(\gamma a K_{0}\right)\right) \cong c \operatorname{Lk}\left(v ; \eta K, \eta K_{0}\right)$.

Proof. Let $h: x \operatorname{Lk}(v ; \eta K) \rightarrow x \operatorname{Lk}(v ; \eta K)$ rel Lk $(v ; \eta K)$ unknot $\psi \phi^{-1}$, by an inductive application of Theorem 4. Then $h \psi \mid \operatorname{Lk}\left(v ; \eta f\left(\gamma a K_{0}\right)\right)=\phi$ and so $h \psi$ is the required homeomorphism of pairs.

This completes the proof of LC and hence completes the induction for Theorem 4.

The most important corollaries of Theorem 4 are local unknottedness criteria in the codimension $\geqq 3$ situation.

Definition 7. We will say that a complex pair $\left(K, K_{0}\right)$ preserves intrinsic skeleta if it satisfies the following equivalent conditions:

a. For $x, y \in K_{0}, x \sim y$ in $K_{0}$ implies $x \sim y$ in $K$.

b. For $A<B$ in $K_{0}$, then $A$ is a nice face of $B$ in $K_{0}$ implies $A$ is a nice face of $B$ in $K$.

c. For $A$ a simplex of $K_{0}, S\left(\operatorname{Lk}\left(A ; K_{0}\right)\right) \subset S(\operatorname{Lk}(A ; K))$.

Proof of equivalence. $\mathrm{a} \rightleftarrows \mathrm{b}$ because $x \sim y$ in $K_{0}$ implies there exists a sequence $A_{0}, \ldots, A_{n}$ with $x \in \operatorname{Int} A_{0}, y \in \operatorname{Int} A_{n}$ and $A_{i}$ a nice face of $A_{i+1}$ or $A_{i+1}$ a nice face of $A_{i}$ for each $i$.

$\mathrm{b} \rightleftarrows \mathrm{c}$ by Corollary II.17a.

Remark. Part a of the above definition is obviously p.l. invariant. So we will say a polyhedral pair $\left(X, X_{0}\right)$ preserves intrinsic skeleta if any triangulation of $\left(X, X_{0}\right)$ does. 
THEOREM 8. Let $\left(X, X_{0}\right)$ be a codimension $\geqq 3$ pair. If $\left(X, X_{0}\right)$ preserves intrinsic skeleta, then $\left(X, X_{0}\right)$ is locally unknotted.

Note that preserving intrinsic skeleta is much weaker, in general, than local unknottedness. See Theorem II.23. In particular, if $X$ is a manifold and $X_{0}<X-\partial X$, then $\left(X, X_{0}\right)$ clearly preserves intrinsic skeleta.

Proof. Let $\left(K, K_{0}\right)$ triangulate the pair $\left(X, X_{0}\right)$. To show that $\left(X, X_{0}\right)$ is locally unknotted it suffices, by Theorem II.23, to show that $A$ is a nice face of $B$ in $K_{0}$ implies $A$ is a nice face of $B$ in $\left(K, K_{0}\right)$. Clearly, it suffices to show this where $\operatorname{dim} B=\operatorname{dim} A+1$, i.e. $B=b A$. So we will show that in this case

$$
\operatorname{Lk}\left(A ; K, K_{0}\right) \cong \Sigma \operatorname{Lk}\left(B ; K, K_{0}\right) .
$$

Since $\left(K, K_{0}\right)$ preserves intrinsic skeleta, $A$ is a nice face of $B$ in $K$. Hence, by Lemma II.14b, there exist, for $\eta_{B} K$ a derived $\bmod B$, homeomorphisms

$$
\begin{gathered}
\phi: \operatorname{Lk}\left(A ; \eta_{B} K_{0}\right) \rightarrow(b+x) \operatorname{Lk}\left(B ; \eta_{B} K_{0}\right) \operatorname{rel} b \operatorname{Lk}\left(B ; \eta_{B} K_{0}\right) \\
\psi: \operatorname{Lk}\left(A ; \eta_{B} K\right) \rightarrow(b+x) \operatorname{Lk}\left(B ; \eta_{B} K\right) \operatorname{rel} b \operatorname{Lk}\left(B ; \eta_{B} K\right)
\end{gathered}
$$

By Lemma 3, it suffices to prove that $\psi \phi^{-1}$ is an unknotted embedding. To do this it suffices to show that $\psi \phi^{-1}$ satisfies the hypotheses of Theorem 4, embedding $x \operatorname{Lk}\left(B ; \eta_{B} K_{0}\right)$ in $x \operatorname{Lk}\left(B ; \eta_{B} K\right)$.

$\mathrm{Lk}\left(B ; \eta_{B}\left(K, K_{0}\right)\right)$ is a codimension $\geqq 3$ pair by Lemma 2 .

Lk $\left(A ; \eta_{B}\left(K, K_{0}\right)\right)$ preserves intrinsic skeleta by Corollary II.12b. Hence, so does $\left((b+x) \operatorname{Lk}\left(B ; \eta_{B} K\right), \psi \phi^{-1}(b+x) \operatorname{Lk}\left(B ; \eta_{B} K_{0}\right)\right)$.

$\phi^{-1}(x) \in S\left(\operatorname{Lk}\left(A ; \eta_{B} K_{0}\right)\right) \subset S\left(\operatorname{Lk}\left(A ; \eta_{B} K\right)\right)$ by part c of Definition 7. Hence,

$$
\psi \phi^{-1}(x) \in S\left((b+x) \operatorname{Lk}\left(B ; \eta_{B} K\right)\right) \cap x \operatorname{Lk}\left(B ; \eta_{B} K\right)=x S\left(\operatorname{Lk}\left(B ; \eta_{B} K\right)\right) .
$$

Finally, if $y=t x+(1-t) y_{1}$ with $0<t<1$ and $y_{1} \in \operatorname{Lk}\left(B ; \eta_{B} K_{0}\right)$. Then, clearly, $y \sim y_{1}$ in $(b+x) \mathrm{Lk}\left(B ; \eta_{B} K\right)$ and in $(b+x) \mathrm{Lk}\left(B ; \eta_{B} K_{0}\right)$. The latter implies that $\psi \phi^{-1} y \sim \psi \phi^{-1} y_{1}=y_{1}$ in $\psi \phi^{-1}(b+x) \operatorname{Lk}\left(B ; \eta_{B} K_{0}\right)$ and since the pair

$$
\left((b+x) \operatorname{Lk}\left(B ; \eta_{B} K\right), \psi \phi^{-1}(b+x) \operatorname{Lk}\left(B ; \eta_{B} K_{0}\right)\right)
$$

preserves intrinsic skeleta, it follows that $\psi \phi^{-1} y \sim y_{1}$ in $(b+x) \operatorname{Lk}\left(B ; \eta_{B} K\right)$. Hence

$$
\begin{aligned}
d\left(y ; x \operatorname{Lk}\left(B ; \eta_{B} K\right)\right) & =d\left(y ;(b+x) \operatorname{Lk}\left(B ; \eta_{B} K\right)\right) \\
& =d\left(\psi \phi^{-1}(y) ;(b+x) \operatorname{Lk}\left(B ; \eta_{B} K\right)\right) \\
& =d\left(\psi \phi^{-1}(y) ; x \operatorname{Lk}\left(B ; \eta_{B} K\right)\right) .
\end{aligned}
$$

Thus, by Lemma 5, $\psi \phi^{-1}$ satisfies the hypotheses of Theorem 4 and the proof is complete.

As immediate corollaries we have the Lickorish unknotting theorems $[\mathbf{L}]$ :

COROllaRY 9. a. Let $f: a X \rightarrow a S$ be a proper embedding of a cone into a ball with $\operatorname{dim} X \leqq \operatorname{dim} S-3$, then $f$ is unknotted. 
b. Let $\left(X, X_{0}\right)$ be a polyhedral pair with $X$ a manifold without boundary and $\operatorname{dim} X_{0} \leqq \operatorname{dim} X-3$, then $\left(X, X_{0}\right)$ is locally unknotted.

Proof. a follows from Theorem 4 and $b$ from Theorem 8 .

VI. Covering isotopies. The Hudson-Zeeman approach to covering isotopies, which we generalize, is based on a close examination of collaring phenomena. So we begin with a theorem listing equivalents of collaring for pairs.

Definition 1. An inclusion $\left(X, X_{0}\right)<\left(Y, Y_{0}\right)$ of polyhedral pairs is called collared if there exists an embedding $h:\left(X, X_{0}\right) \times I \rightarrow\left(Y, Y_{0}\right)$ with $h^{-1} Y_{0}=X_{0} \times I, h_{0}=$ the inclusion $X \rightarrow Y$ and $H(X \times I)$ is a neighborhood of $X$ in $Y$.

The inclusion is called locally collared if for each point $x \in X_{0}$ there is a neighborhood $N$ of $x$ in $Y$ such that $\left(N \cap X, N \cap X_{0}\right)<\left(N, N \cap Y_{0}\right)$ is collared and for each point $x \in X-X_{0}$ there is a neighborhood $N$ of $x$ in $Y$ such that $N \cap X<N$ is collared.

The following theorem is a straightforward generalization to pairs of $[\mathbf{C}$; Proposition 4.2].

Note that the equivalence of a and $d$ was used in the proof of Theorem V.4.

THEOREM 2. For an inclusion of complex pairs: $i:\left(K, K_{0}\right) \subset\left(J, J_{0}\right)$ with $K_{0}=$ $J_{0} \cap K$, and $K$ full in $J$, the following are equivalent:

a. There exists a homeomorphism

$$
h:\left(J, J_{0}\right) \cup\left(K, K_{0}\right) \times I \rightarrow\left(J, J_{0}\right)
$$

(where in the union $x \in K$ is identified with $(x, 0) \in K \times I$ ) such that $h_{1}: K \rightarrow J$ is the inclusion.

b. $i$ is collared.

c. $i$ is locally collared.

d. For $x \in K_{0}, \operatorname{Lk}\left(x ; J, J_{0}\right) \cong c \operatorname{Lk}\left(x ; K, K_{0}\right) \operatorname{rel~Lk}(x ; K)$ and for $x \in K-K_{0}$, $\mathrm{Lk}(x ; J) \cong c \operatorname{Lk}(x ; K) \operatorname{rel} \mathrm{Lk}(x ; K)$.

e. For $A \in K_{0}, \operatorname{Lk}\left(A ; J, J_{0}\right) \cong c \operatorname{Lk}\left(A ; K, K_{0}\right) \operatorname{rel} \operatorname{Lk}(A ; K)$ and for $A \in K-K_{0}$, $\mathrm{Lk}(A ; J) \cong c \operatorname{Lk}(A ; K) \operatorname{rel} \operatorname{Lk}(A ; K)$.

f. Let $\eta J$ be a derived of $J$ and for $A \in K$ let $C(A ; J)=\dot{N}(D(A ; K) ; D(A ; J))$ $=C(\dot{D}(A ; K) ; \dot{D}(A ; J))$ and $\dot{C}(A ; J)=\dot{N}(\dot{D}(A ; K) ; \dot{D}(A ; J))$ and similarly for $A \in K_{0}$ define $C\left(A ; J_{0}\right)$ and $\dot{C}\left(A ; J_{0}\right)$. By convention, for $A \in K-K_{0}$, let $C\left(A ; J_{0}\right)$ $=\dot{C}\left(A ; J_{0}\right)=\varnothing$.

Then there exists a homeomorphism

$$
g: N\left(K ; \eta\left(J, J_{0}\right)\right) \rightarrow\left(K, K_{0}\right) \times I \operatorname{rel} K
$$

and such that:

1. $g\left(D\left(A ; J, J_{0}\right)\right)=D\left(A ; K, K_{0}\right) \times I$ for $A \in K$.

2. $g\left(C\left(A ; J, J_{0}\right)\right)=D\left(A ; K, K_{0}\right) \times 1$ for $A \in K$.

Proof. $a \rightarrow b \rightarrow c \rightarrow d$ are clear. $d \rightarrow e$ is a straightforward restatement of Cohen's argument in the proof of [C; Proposition 4.2] for pairs. $f \rightarrow a$ is simply a 
matter of pulling the external collar into $\left(J, J_{0}\right)$ by shrinking the internal collar given by $f$.

The proof that $\mathrm{e} \rightarrow \mathrm{f}$ is also an easy restatement of the corresponding portion of [C; Proposition 4.2] for pairs, but because its argument is crucial to our later proofs, we will outline it.

The first step is to obtain for each $A \in K$ a homeomorphism

$$
\phi_{A}: C\left(A ; J, J_{0}\right) \rightarrow c \dot{C}\left(A ; J, J_{0}\right) \operatorname{rel} \dot{C}(A ; J) .
$$

Using the canonical isomorphism

$$
\dot{D}\left(A ; J, J_{0}\right) \rightarrow \eta \operatorname{Lk}\left(A ; J, J_{0}\right)
$$

which takes, by naturality, $\dot{D}\left(A ; K, K_{0}\right) \rightarrow \eta \mathrm{Lk}\left(A ; K, K_{0}\right)$, we get from e a homeomorphism $\dot{D}\left(A ; J, J_{0}\right) \cong c \dot{D}\left(A ; K, K_{0}\right)$ which takes $N\left(\dot{D}(A ; K) ; \dot{D}\left(A ; J, J_{0}\right)\right)$ to a regular neighborhood of $\dot{D}\left(A ; K, K_{0}\right)$ in its cone pair. Uniqueness of regular neighborhoods and their complements gives the homeomorphism $\phi_{A}$, exhibiting $C\left(A ; J, J_{0}\right)$ as the cone on $\dot{C}\left(A ; J, J_{0}\right)$, the boundary pair.

We decompose $N\left(K ; \eta J, \eta J_{0}\right)$ in a "complex" of cone pairs indexed by the simplices of $K$, namely:

$$
N\left(K ; \eta J, \eta J_{0}\right)=\bigcup\left\{D\left(A ; J, J_{0}\right): A \in K\right\} \cup \bigcup\left\{C\left(A ; J, J_{0}\right): A \in K\right\}
$$

with $D\left(A ; J, J_{0}\right)=\eta(A) * \dot{D}\left(A ; J, J_{0}\right)$ and $C\left(A ; J, J_{0}\right) \cong c \dot{C}\left(A ; J, J_{0}\right)$. Furthermore,

$$
\dot{D}\left(A ; J, J_{0}\right)=\bigcup\left\{D\left(B ; J, J_{0}\right)=A<B \in \mathrm{K}\right\} \cup C\left(A ; J, J_{0}\right)
$$

and

$$
\dot{C}\left(A ; J, J_{0}\right)=\bigcup\left\{C\left(B ; J, J_{0}\right): A<B \in K\right\} .
$$

Furthermore, distinct cone-pairs intersect in the intersection of the base pairs.

Similarly, we can decompose

$$
\left(K, K_{0}\right) \times I=\bigcup\left\{D\left(A ; K, K_{0}\right) \times I: A \in K\right\} \cup \bigcup\left\{D\left(A ; K, K_{0}\right) \times 1: A \in K\right\} .
$$

$D\left(A ; K, K_{0}\right) \times 1=(\eta(A), 1) * \dot{D}\left(A ; K, K_{0}\right) \times 1$ and by the Alexander trick there is an isomorphism $D\left(A ; K, K_{0}\right) \times I \cong(\eta(A), 0) *\left(\dot{D}\left(A ; K, K_{0}\right) \times I \cup D\left(A ; K, K_{0}\right) \times 1\right)$ which is the identity mapping $D\left(A ; K, K_{0}\right) \times 0$ to $(\eta(A), 0) * \dot{D}\left(A ; K, K_{0}\right) \times 0$.

We associate the "complexes" by a map $\gamma: \gamma\left(D\left(A ; J, J_{0}\right)\right)=D\left(A ; K, K_{0}\right) \times I$ and $\gamma\left(C\left(A ; J, J_{0}\right)\right)=D\left(A ; K, K_{0}\right) \times 1 . \gamma$ clearly preserves incidences and so we can construct a homeomorphism, $g$, of polyhedral pairs which is carried by $\gamma$ and extends the identity mapping $\left(K, K_{0}\right) \rightarrow\left(K, K_{0}\right) \times 0$. Just go by induction up an ordering of the cone pairs of the domain by increasing dimension and at each stage extend $g$ by coning.

We now prove the key lemma, sharpening Theorem 2, and crucial to the covering isotopy theorem. 
LEMMA 3. Let $F: X \times I \rightarrow Y \times I$ be an embedding with $F^{-1}(Y \times \cdot 0)=X \times 0$ and satisfying:

a. $(Y \times 0, F(X \times 0))<(Y \times I, F(X \times I))$ is locally collared.

b. For every $x \in X, d(F(x, t) ; Y \times I, F(X \times I))$ is constant for $0<t<1$.

Then there exists a regular neighborhood $V(X)$ of $X$ in $X \times I$, regular neighborhoods $V(Y)$ and $V_{0}(Y)$ of $Y$ in $Y \times I$, and collaring homeomorphisms $g: X \times I \rightarrow V(X)$ rel $X$ $=X \times 0, G: Y \times I \rightarrow V(Y)$ and $G_{0}: Y \times I \rightarrow V_{0}(Y)$ rel $Y=Y \times 0$ such that the following diagram commutes:

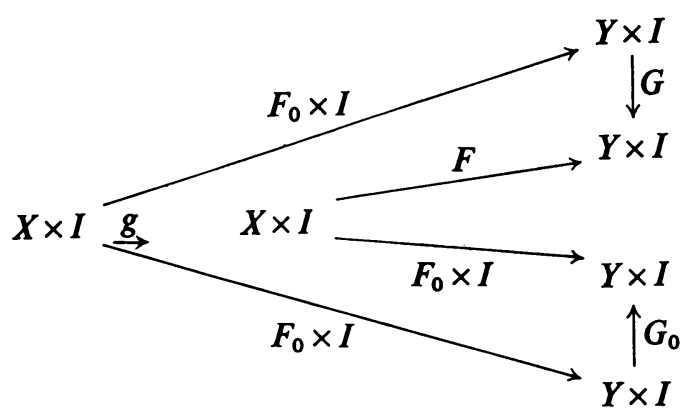

Note. The importance of this lemma is to collar $F(X \times I)$ and $F_{0}(X) \times I$ in $Y \times I$ and induce the same collaring of $X \times I$.

Proof. By identifying $X$ with $F_{0}(X)$ by $F_{0}$ and identifying $F: X \times I \rightarrow Y \times I$ with $F \cdot\left(F_{0} \times I\right)^{-1}: F_{0}(X) \times I \rightarrow Y \times I$, we can assume that $X<Y$ and $F_{0}$ is the inclusion. Then by triangulating and subdividing to make the diagram simplicial,

$$
Y \times 0 \stackrel{\text { proj }}{\longleftarrow} Y \times I \stackrel{\text { inc }}{\longleftarrow} X \times I \cup Y \times 0 \stackrel{F \cup \text { inc }}{\longrightarrow} Y \times I
$$

we obtain the simplicial situation of a complex pair $(J, K)$ and a simplicial map $F: \alpha(K \times I) \rightarrow \beta(J \times I)$ with $\alpha(K \times I)$ a subcomplex of $\alpha(J \times I)$ which is a cylindrical subdivision. Furthermore, we can assume $\alpha(K \times 0)=K \times 0=K$ and $\alpha(J \times 0)=J \times 0$ $=\beta(J \times 0)=J$. Our assumptions on $F$ become

1. $(J \times 0, K \times 0)<(J \times I, F(K \times I))$ is locally collared.

2. For $x \in K, d(F(x, t) ; J \times I, F(K \times I))$ is constant for $0<t<1$. This clearly implies, since $\alpha(K \times I)$ is cylindrical with $\alpha(K \times 0)=K \times 0$, that for $A \in K, d(F(y)$; $J \times I, F(K \times I))$ is constant for $y \in \operatorname{Int} A \times I$.

Now let $(V(J), V(K))=N(J \times 0 ; \eta \beta(J \times I), \eta f(\alpha(K \times I)))$ and $\left(V_{0}(J), V_{0}(K)\right)$ $=N(J \times 0 ; \eta \alpha(J \times I), \eta \alpha(K \times I))$ where $\eta \beta J \times I$ and $\eta \alpha J \times I$ are deriveds chosen so that $F: \eta \alpha K \times I \rightarrow \eta \beta J \times I$ is simplicial.

Following Theorem $2 \mathrm{f}$ we will construct homeomorphisms $G:(J, K) \times I \rightarrow$ $(V(J), V(K))$ and $G_{0}:(J, K) \times I \rightarrow\left(V_{0}(J), V_{0}(K)\right)$ rel $J=J \times 0$, and such that $G \mid K \times I=F\left(G_{0} \mid K \times I\right)$. If we then define $g=G_{0} \mid K \times I$ we will be done. 
We decompose $\left(V_{0}(J), V_{0}(K)\right)$ into a cone pair complex, as in the proof of Theorem 2, consisting of pairs

$$
\{C(A ; \alpha J \times I, \alpha K \times I): A \in J\} \quad \text { and } \quad\{D(A ; \alpha J \times I, \alpha K \times I): A \in J\} .
$$

Similarly, decompose $(V(J), V(K))$ into $\{C(A ; \beta J \times I, F(\alpha K \times I)): A \in J\}$ and $\{D(A ; \beta J \times I, F(\alpha K \times I)): A \in J\}$ and $(J, K) \times I$ into $\{D(A ; J, K) \times 1: A \in J\}$ and $\{D(A ; J, K) \times I: A \in J\}$.

We first reduce the proof to the following technical assertion:

ASSERTION. For $A \in K$ there exist homeomorphisms

$$
\begin{aligned}
\phi_{A}^{0}: C(A ; \alpha J \times I, \alpha K \times I) & \rightarrow c \dot{C}(A ; \alpha J \times I, \alpha K \times I) \text { rel } \dot{C}, \\
\phi_{A}: C(A ; \beta J \times I, F(\alpha K \times I)) & \rightarrow c \dot{C}(A ; \beta J \times I, F(\alpha K \times I)) \text { rel } \dot{C},
\end{aligned}
$$

such that $\left(c * F\left(\phi_{A}^{0} \mid C(A ; \alpha K \times I)\right)=\left(\phi_{A} \mid C(A ; F(\alpha K \times I))\right) F\right.$.

Note that since the dual cells are defined in terms of $\eta \beta J \times I$ and $\eta \alpha J \times I$ and $F: \eta \alpha K \times I \rightarrow \eta \beta J \times I$ is simplicial, the restriction of $F$ maps

$$
(C(A ; \alpha K \times I), \dot{C}(A ; \alpha K \times I))
$$

to $(C(A ; F(\alpha K \times I)), \dot{C}(A ; F(\alpha K \times I)))$ by a simplicial isomorphism.

Now for $A \in J-K$ there exist homeomorphisms

$$
\begin{aligned}
& \phi_{A}^{0}: C(A ; \alpha J \times I) \rightarrow c \dot{C}(A ; \alpha J \times I) \text { rel } \dot{C}, \\
& \phi_{A}: C(A ; \beta J \times I) \rightarrow c \dot{C}(A ; \beta J \times I) \text { rel } \dot{C} .
\end{aligned}
$$

Define $G:(J, K) \times I \rightarrow(V(J), V(K))$ and $G_{0}:(J, K) \times I \rightarrow\left(V_{0}(J), V_{0}(K)\right)$ to be carried respectively by $\gamma$ and $\gamma_{0}$, where $\gamma(D(A ; J, K) \times I)=D(A ; \beta J \times I, F(\alpha K \times I))$ and $\gamma_{0}(D(A ; J, K) \times I)=D(A ; \alpha J \times I, \alpha K \times I)$;

$$
\gamma(D(A ; J, K) \times 1)=C(A ; \beta J \times I, F(\alpha K \times I))
$$

and

$$
\gamma_{0}(D(A ; J, K) \times 1)=C(A ; \alpha J \times I, \alpha K \times I) .
$$

Using $\phi_{A}$ and $\phi_{A}^{0}$ we define the conical extensions over the $C(A)$ 's.

Thus, if $G: \dot{D}(A ; J, K) \times 1 \rightarrow \dot{C}(A ; \beta J \times I, F(\alpha K \times I))$ and $G_{0}: \dot{D}(A ; J, K) \times 1$ $\rightarrow \dot{C}(A ; \alpha J \times I, \alpha K \times I)$ are defined so that $G=F G_{0}$ on the intersection with $K \times I$ then define $G$ and $G_{0}$ on $D(A ; J, K) \times 1$ by $\phi_{A}^{-1}(c G)$ and $\phi_{A}^{0^{-1}}\left(c G_{0}\right)$ respectively. Since $F$ on $D(A ; J, K) \times 1$ is the cone of $F$ on $\dot{D}(A ; J, K)$, we have, on $D(A ; K) \times 1$,

$$
\begin{gathered}
F G_{0}=F \phi_{A}^{0-1} c G_{0}=\phi_{A}^{-1} c F c G_{0}, \\
\phi_{A}^{-1} c\left(F G_{0}\right)=\phi_{A}^{-1} c G=G .
\end{gathered}
$$

If $G: \dot{D}(A ; J, K) \times I \cup D(A ; J, K) \times 1 \rightarrow \dot{D}(A ; \beta J \times I, F(\alpha K \times I))$ and

$$
G_{0}: \dot{D}(A ; J, K) \times I \cup D(A ; J, K) \times 1 \rightarrow \dot{D}(A ; \alpha J \times I, \alpha K \times I)
$$

are defined so that $G=F G_{0}$, then the extension by coning using the Alexander trick also satisfies $G=F G_{0}$ on $K \times I$. 
Thus, the proof is reduced to the assertion that the compatible $\phi_{A}$ and $\phi_{A}^{0}$ for $A \in K$ exist.

Claim A. There exists a homeomorphism $\psi: \operatorname{Lk}(A ; \alpha J \times I) \rightarrow c \operatorname{Lk}(A ; J)$ $\operatorname{rel~Lk}(A ; J)$ such that for $B \in \operatorname{Lk}(A ; J), \psi(\operatorname{Lk}(A ; \alpha(A * B) \times I))=c B$.

Proof. As in the proof of Claim B of the proof of Theorem IV.4, we define $S^{i}=A * \operatorname{Lk}(A ; J)^{(i)}$ and define $\psi^{i}: \operatorname{Lk}\left(A ; \alpha S^{i} \times I\right) \rightarrow c \operatorname{Lk}(A ; J)^{(i)}$. Starting with $i=-1, S^{-1}=A, \operatorname{Lk}\left(A ; \alpha S^{-1} \times I\right)=$ one point which is mapped to $c$.

Assume $\psi^{i-1}$ is defined and, for $B$ and $i$-simplex of $\operatorname{Lk}(A ; J)$, define

$$
\psi^{i}: \mathrm{Lk}(A ; \alpha(A * B) \times I) \rightarrow c B
$$

to extend the identity on $B$ and $\psi^{i-1}$ mapping $\operatorname{Lk}(A ; \alpha(A * \dot{B}) \times I) \rightarrow c \dot{B}$. This is just a question of extending a homeomorphism of the boundaries of two balls to the balls.

The induction completed, let $\psi=\psi^{i}$ for $i=\operatorname{dim} \operatorname{Lk}(A ; J)$.

Claim B. There exists a homeomorphism $\phi^{0}: C(A ; \alpha J \times I) \rightarrow c \dot{C}(A ; \alpha J \times I)$ rel $\dot{C}$ such that, for $D \in \mathrm{St}^{\circ}(A ; J), \phi^{0} C(A ; \alpha D \times I)=c C^{\prime}(A ; \alpha D \times I)$. In particular, $\phi^{\circ} C(A ; \alpha K \times I)=c C^{\prime}(A ; \alpha K \times I)$.

Proof. Using $\psi$ as defined in Claim A, and using the canonical isomorphism $j: \dot{D}(A ; \alpha J \times I) \rightarrow \eta \operatorname{Lk}(A ; \alpha J \times I)$ we obtain a homeomorphism

$$
\dot{D}(A ; \alpha J \times I) \rightarrow c \dot{D}(A ; J) \text { rel } \dot{D}(A ; J)
$$

which takes $N\left(D(A ; J) ; D(A ; \alpha J \times I),\left\{D\left(A ; \alpha D_{j} \times I\right)\right\}\right)$ to a regular neighborhood of $D(A ; J)$ in $\left(c D(A ; J),\left\{c D\left(A ; D_{j}\right)\right\}\right)$ where $\left\{D_{j}\right\}$ is the set of simplices in $\mathrm{St}^{\circ}(A ; J)$. Uniqueness of regular neighborhood families and their complements, applied to $\dot{D}(A ; J)$ in the cone family, gives the required homeomorphism.

Claim C. $F \phi^{0^{-1}}: c \dot{C}(A ; \alpha K \times I) \rightarrow \dot{D}(A ; \beta J \times I)$ is $\mathrm{CH}$.

Proof. For $y \in C(A ; F(\alpha K \times I))-\dot{C}(A ; F(\alpha K \times I))$.

$$
\begin{aligned}
d(y ; \dot{D}(A ; \beta J \times I), C(A ; F(\alpha K \times I))) & =d(y ; \dot{D}(A ; \beta J \times I, F(\alpha K \times I))) \\
& =d(y ; J \times I, F(K \times I))-\operatorname{dim} A-1
\end{aligned}
$$

by Lemma II.19. Since

$$
F \phi^{0^{-1}}(C(A ; \alpha K \times I)-\dot{C}(A ; \alpha K \times I))=C(A ; F(\alpha K \times I))-\dot{C}(A ; F(\alpha K \times I)),
$$

it follows that for $x \in \dot{C}(A ; \alpha K \times I)$ it suffices to prove that

$$
d\left(F \phi^{0-1}(t c+(1-t) x) ; J \times I, F(K \times I)\right)
$$

is constant for $0<t<1$.

There is a unique $D \in \mathrm{St}^{\circ}(A ; K)$ such that $x \in \dot{C}(A ; \alpha D \times I)-\dot{C}(A ; \alpha \dot{D} \times I)$. By Claim B, $\phi^{0-1}(t c+(1-t) x) \in C(A ; \alpha D \times I)-C(A ; \alpha \dot{D} \times I)$ for all $0<t<1$.

Hence,

$$
C(A ; \alpha D \times I)-C(A ; \alpha \dot{D} \times I) \subset \text { Int } D \times I .
$$

$$
d\left(F \phi^{0-1}(t c+(1-t) x) ; J \times I, F(K \times I)\right)
$$

is constant for $0<t<1$, by the intrinsic skeleton assumption on $F$. 
Proof of the Assertion. Since $(J \times I, F(K \times I))$ is locally collared, $C(A ; \beta J \times I)$ is a regular neighborhood of $C(A ; F(\alpha K \times I)) \bmod \dot{C}(A ; F(\alpha K \times I))$ in $\dot{D}(A ; \beta J \times I)$. For by the proof of Theorem $2 C(A ; \beta J \times I, F(\beta K \times I))$ is homeomorphic to the cone pair on $\dot{C}(A ; \beta J \times I, F(\alpha K \times I))$ and is the complement of a regular neighborhood pair in $\dot{D}(A ; \beta J \times I)$ and hence is locally collared on the outside. So we can apply Theorem III.11c or the stellar neighborhood theorem to get this regular neighborhood fact.

Theorem IV.4 now implies that there exists a homeomorphism

$$
\phi: C(A ; \beta J \times I) \rightarrow c \dot{C}(A ; \beta J \times I)
$$

rel $\dot{C}$ and such that

$$
\phi F \phi^{0^{-1}}=\overline{\left(F \phi^{0-1}\right)}=c F .
$$

So let $\phi_{A}=\phi$ and $\phi_{A}^{0}=\phi^{0}$.

With Lemma 3 taken care of, the proof of the covering isotopy follows from a sequence of arguments taken directly from Hudson-Zeeman.

Lemma 4. Let $\left(X, X_{0}\right)$ be a polyhedral pair and $c: X \times[0, \varepsilon] \rightarrow X \times I$ an embedding with $c^{-1}(X \times 0)=X \times 0$ and $c \mid X_{0} \times[0, \varepsilon]$ level-preserving. Then there exists $0<\delta<\varepsilon$ and $h: X \times I \rightarrow X \times I$ a homeomorphism, with hc level-preserving on $X \times[0, \delta]$ and $h$ ambient isotopic to the identity rel $X \times I \cup c\left(X_{0} \times[0, \varepsilon]\right)$.

Proof. Triangulate and subdivide so that $c: \alpha K \times[0, \varepsilon] \rightarrow \beta K \times I$ is simplicial and $K_{0}$ is a subcomplex of $K_{0}$ covering $X_{0}$.

Let $\varepsilon>\delta>0$ be chosen so that no vertex of $\alpha K \times[0, \varepsilon]$ or $K \times I$ has level $0<t \leqq \delta$, and such that $c(K \times[0, \delta]) \cap K \times 1=\varnothing$.

Let $\eta \alpha K \times[0, \varepsilon]$ be a derived with $\eta A \in$ Int $A \cap K \times \delta$ if Int $A \cap K \times \delta \neq \varnothing$. Let $\eta_{0} \beta K \times I$ be a derived such that $c: \eta \alpha K \times[0, \varepsilon] \rightarrow \eta_{0} \beta K \times I$ is simplicial and let $\eta_{1} \beta K \times I$ agree with $\eta_{0} \beta K \times I$ except for

$$
c(N(K \times 0 ; \alpha K \times[0, \varepsilon]))-c\left(K_{0} \times[0, \varepsilon] \cup K \times 0\right)
$$

and on these simplices, let $\eta_{1}(A) \in \operatorname{Int} A \cap K \times \delta$. Note that if

$$
A \in c N\left(K_{0} \times 0 ; \alpha\left(K_{0} \times[0, \varepsilon]\right)\right)-K \times 0, \eta_{1}(A)=\eta_{0}(A) \in K \times \delta
$$

since $c$ is level preserving on $K_{0} \times[0, \varepsilon]$.

Let $h: \eta_{0} \beta K \times I \rightarrow \eta_{1} \beta K \times I$ be defined by $h\left(\eta_{0} A\right)=\eta_{1} A$.

REMARK. Note that if $X_{1}<X$ and then $h$ can be assumed a homeomorphism of pairs $\left(X \times I, c\left(X_{1} \times[0, \varepsilon]\right)\right)$.

We now cover an isotopy locally:

LemmA 5. Given $X, Y$ compact polyhedra let $F: X \times I \rightarrow Y \times I$ be an isotopy satisfying:

1. $\left(Y \times 0, F_{0}(X) \times 0\right) \subset(Y \times I, F(X \times I))$ is locally collared.

2. For every $x \in X, d(F(x, t) ; Y \times I, F(X \times I))$ is constant for $0<t<1$. 
Then, there exists $\varepsilon>0$ and a homeomorphism $h: Y \times[0, \varepsilon] \rightarrow Y \times[0, \varepsilon]$ rel $Y \times 0$ and level-preserving such that: $h\left(F_{0} \times[0, \varepsilon]\right)=F \mid X \times[0, \varepsilon]$.

Proof. Choose compatible collarings $g, G_{0}$ and $G$ by Lemma 3. By compactness, we can choose $\delta>0$ so that $Y \times[0, \delta] \subset G_{0}(Y \times I)$.

Define $h_{0}: Y \times[0, \delta] \rightarrow Y \times I$ by $G G_{0}^{-1}\left|Y \times[0, \delta] . h_{0}\right| Y \times 0=$ identity and by compatibility, $h_{0} \mid F_{0}(X) \times[0, \delta]=F\left(F_{0} \times[0, \delta]\right)^{-1}$, and, in particular, is levelpreserving on $F_{0}(X) \times[0, \delta]$.

By Lemma 4, there exists $0<\delta^{\prime}<\delta$ and $h_{1}: Y \times I \rightarrow Y \times I$ rel $Y \times 0 \cup F(X \times[0, \delta])$ and $h_{1} h_{0}=h$ is level-preserving on $Y \times\left[0, \delta^{\prime}\right]$.

Now $h\left(Y \times \delta^{\prime}\right) \subset Y \times \delta^{\prime}$ but might be proper, we note that $h\left(Y \times\left[0, \delta^{\prime}\right]\right)$ is a neighborhood of $Y \times 0$ and hence, by compactness, there exists $0<\varepsilon \leqq \delta^{\prime}$ with $Y \times[0, \varepsilon] \subset h\left(Y \times\left[0, \delta^{\prime}\right]\right)$. So $h \mid Y \times[0, \varepsilon]$ being level-preserving, is a homeomorphism.

Definition 6 (FOllowing RouRKe [R]). We call an isotopy $F: X \times I \rightarrow Y \times I$ locally collared if for every subinterval $J$ of $I,(Y \times \dot{J}, F(X \times \dot{J})) \subset(Y \times J, F(X \times J))$ is locally collared, or equivalently $(Y \times t, F(X \times t)) \subset(Y \times[t, 1], F(X \times[t, 1]))$ is locally collared for all $0 \leqq t<1$ and $(Y \times t, F(X \times t)) \subset(Y \times[0, t], F(X \times[0, t]))$ is locally collared for all $0<t \leqq 1$.

TheOREM 7. Let $F: X \times I \rightarrow Y \times I$ be an isotopy, with $X$ compact. Then a necessary and sufficient condition that $F$ can be covered by an ambient isotopy of $Y$ is that

1. $F$ be locally collared, and,

2. for every $x \in X, d(F(x, t) ; Y \times I, F(X \times I))$ is constant for $0<t<1$.

Proof. Necessity is clear. To prove sufficiency, we lift the compactness argument from Hudson-Zeeman, assuming $Y$ is compact also.

Let $t \in I$, then by Lemma 5, applied in both directions, there exists a subinterval $J_{t}$ with $t \in \operatorname{Int}_{I} J_{t}$ and a homeomorphism

$$
h(t): Y \times J_{t} \rightarrow Y \times J_{t}
$$

with $h(t)$ level-preserving and $h(t)\left(F_{t} \times J_{t}\right)=F \mid X \times J_{t}$.

By compactness of $I$, there exists a sequence $0=s_{0}<s_{1}<\cdots<s_{n}=1$ such that for each $i=1, \ldots, n$, there exists $t_{i}$ and $J_{i}=J_{t_{i}}$ with $\left[s_{i-1}, s_{i}\right] \subset \operatorname{Int}_{I} J_{i}$, let $\left[0, s_{0}\right]=J_{0}$.

Inductively, we define $H(i): Y \times\left[0, s_{i}\right] \rightarrow Y \times\left[0, s_{i}\right]$ rel $Y \times 0$ and level-preserving homeomorphisms, such that $H(i)\left(F_{0} \times\left[0, s_{i}\right]\right)=F \mid X \times\left[0, s_{i}\right]$. Let $H(0)=h(0)$.

$$
\begin{aligned}
H(i) & =H(i-1) \quad \text { on } Y \times\left[0, s_{i-1}\right] \\
& =h\left(t_{i}\right)\left(h\left(t_{i}\right)_{s_{i-1}} \times\left[s_{i-1}, s_{i}\right]\right)^{-1}\left(H(i-1)_{s_{i-1}} \times\left[s_{i-1}, s_{i}\right]\right) \quad \text { on } Y \times\left[s_{i-1}, s_{i}\right] .
\end{aligned}
$$

Let $H=H(n): Y \times I \rightarrow Y \times I$ rel $Y \times 0$. This is the required ambient isotopy.

In the case that $Y$ is not compact, we let $Y^{*}=$ a regular neighborhood of $\operatorname{proj}_{Y} F(X \times I)$ and let $X^{*}=X \cup$ boundary of the regular neighborhood. Let $F^{*}=F$ on $X \times I$ and the identity on the boundary. If $H^{*}$ is the ambient isotopy of $Y^{*}$, let $H$ extend $H^{*}$ by the identity on the rest of $Y \times I$. 
We outline a related theorem of Rourke [R]:

Definition 8. If $F: X \times I \rightarrow Y \times I$ is an isotopy and $G: Y \times I \rightarrow Y \times I$ is an ambient isotopy, we shall say that $G$ covers the track of $F$, if $G\left(F_{0}(X)\right)=F_{t}(X)$.

TheOREM 9 [R]. Let $F: X \times I \rightarrow Y \times I$ be an isotopy with $X$ and $Y$ compact. Then a necessary and sufficient condition that the track of $F$ can be covered by an ambient isotopy is that $F$ be locally collared.

Proof. Necessity is again clear and for sufficiency it suffices to cover the track of $F \mid X \times[0, \varepsilon]$ for some $\varepsilon$, as the result then follows by a compactness argument identical with Theorem 7.

By local collaring, there is a homeomorphism

$$
G:\left(Y \times I, F_{0}(X) \times I\right) \rightarrow(Y \times I, F(X \times I)) \text { rel } Y \times 0 .
$$

By Lemma 4 and the remark thereafter, there exists a homeomorphism

$$
h:(Y \times I, F(X \times I)) \rightarrow(Y \times I, F(X \times I)) \text { rel } Y \times 0
$$

and a $1>\delta>0$, with $G^{*}=h G$ level-preserving on $Y \times[0, \delta]$, and so there exists $0<\varepsilon \leqq \delta$ such that $G^{*}(Y \times[0, \varepsilon])=Y \times[0, \varepsilon]$ and $G^{*}\left(F_{0}(X) \times[0, \varepsilon]\right)=F(X \times[0, \varepsilon])$. Then $G^{*} \mid Y \times[0, \varepsilon]$ is the required local covering.

REMARK. As in Theorem 7, it is possible to get rid of the assumption that $Y$ is compact.

Corollary 10. Let $\left(X, X_{0}\right)$ be a polyhedral pair with $X_{0}$ compact, and let $F: X_{0} \times I \rightarrow X_{0} \times I$ be an ambient isotopy of $X_{0}$. Then $F$ can be covered by an ambient isotopy of $X$ iff $F\left(I^{i}\left(X, X_{0}\right) \times I\right)=I^{i}\left(X, X_{0}\right) \times I$.

Proof. Again necessity is clear. For sufficiency, note that $F$ is obviously locally collared (regarded as an isotopy in $X$ ), and in the presence of local collaring, $d\left(F(x, t) ; X \times I, F\left(X_{0} \times I\right)\right)=d\left(F(x, t) ; X \times t, F\left(X_{0} \times t\right)\right)+1$, which is in this case $d\left(F_{t}(x) ; X, X_{0}\right)+1$ which is constant for $0 \leqq t \leqq 1$ by hypothesis.

Corollary 11. Let $X$ be a compact polyhedron, and $F: I^{i}(X) \times I \rightarrow I^{i}(X) \times I$ be an ambient isotopy of $I^{i}(X)$. Then $F$ can be covered by an ambient isotopy of $X$ iff $F\left(I^{j}(X) \times I\right)=I^{j}(X) \times I$ for all $j \leqq i$.

Proof. Corollary 10 and Corollary II.21 imply the result.

Corollary 12. Let $X_{0}, Y<X$ be polyhedra with $X_{0}$ compact, and $F: X_{0} \times I$ $\rightarrow X_{0} \times I$ an ambient isotopy of $X_{0}$ rel $X_{0} \cap Y$. If $F$ can be covered by an ambient isotopy of $X$, then there exists an ambient isotopy of $X$ rel $Y$ covering $F$.

Proof. Assume $X$ compact. Define $F:\left(X_{0} \cup Y\right) \times I \rightarrow\left(X_{0} \cup Y\right) \times I$ to be $F$ on $X_{0} \times I$ and the identity on $Y \times I$.

$$
d\left(\bar{F}_{t}(x) ; X, X_{0} \cup Y\right)=d\left(x ; X, X_{0} \cup Y\right) \text { for all } x \in X_{0} \cup Y \text { and } 0 \leqq t \leqq 1 .
$$


This is clear for $x \in Y$ and for $x \in X_{0}-Y$,

$$
d\left(\bar{F}_{t}(x) ; X, X_{0} \cup Y\right)=d\left(F_{t}(x) ; X, X_{0}\right)=d\left(x ; X, X_{0}\right)=d\left(x ; X, X_{0} \cup Y\right) .
$$

So $\bar{F}$ can be covered by an ambient isotopy by Corollary 10 .

Now we want to introduce local unknottedness conditions instead of the ambient intrinsic dimension conditions.

Definition 13. Call a locally collared isotopy $F: X \times I \rightarrow Y \times I$, with $X$ compact, locally unknotted if it satisfies the following equivalent conditions.

1. $\left(Y, F_{0}(X)\right)$ is locally unknotted.

2. $(Y \times I, F(X \times I))$ is locally unknotted at all points $F(x, t), 0<t<1$.

Proof of the equivalence. Since, by Rourke's theorem, Theorem 9, the track of $F$ can be covered by an ambient isotopy of $Y$ (or a regular neighborhood of $\operatorname{proj}_{Y} F(X \times I)$ if $Y$ is not compact), it follows that $\left(Y, F_{0}(X)\right) \cong\left(Y, F_{t}(X)\right)$ for all $t$.

Now $d(F(x, t) ; Y \times I, F(X \times I))=d\left(F_{t}(x) ; Y, F_{t}(X)\right)+1$ by local collaring and similarly $d(F(x, t) ; F(X \times I))=d\left(F_{t}(x) ; F_{t}(X)\right)+1$, for all $0<t<1$.

Thus, $\left(Y, F_{0}(X)\right)$ is locally unknotted iff $\left(Y, F_{t}(X)\right)$ is locally unknotted for all $0<t<1$ iff $(Y \times I, F(X \times I))$ is locally unknotted at every point $F(x, t)$ with $x \in X$ and $0<t<1$.

REMARK. Note that if $X_{1}<X_{0}<X$ are polyhedra with $\left(X, X_{0}\right)$ locally unknotted at every point of $X_{0}-X_{1}$ and $F: X_{0} \times I \rightarrow X_{0} \times I$ is an ambient isotopy of $X_{0}$ rel $X_{1}$, then $F$ regarded as an isotopy $X_{0} \times I \rightarrow X \times I$ satisfies the hypotheses of Theorem 7 . In particular, we have

COROLlaRY 14. Let $F: X \times I \rightarrow Y \times I$ be a locally unknotted isotopy, then $F$ can be covered by an ambient isotopy of $Y$.

Proof. $d(F(x, t) ; F(X \times I))$ is constant for $0<t<1$ and hence part 2 of Definition 13 and Theorem 7 imply the result.

CoROllary 15. Let $F: X \times I \rightarrow Y \times I$ be an isotopy with $X$ compact and $\left(Y, F_{0}(X)\right)$ locally unknotted. Then if the track of $F$ can be covered by an ambient isotopy of $Y$, $F$ itself can be covered by an ambient isotopy of $Y$.

Proof. Theorem 9 and Corollary 14.

Our notion of locally unknotted isotopy reduces to the usual one in the case of manifolds. We leave to the reader the proof:

Let $F: X \times I \rightarrow Y \times I$ be an isotopy with $X, Y$ manifolds and $X$ compact and such that $F^{-1}(\partial Y \times I)=\partial X \times I$. Then the following are equivalent:

1. $F$ is locally unknotted.

2. For every subinterval $J$ of $I,(Y \times J, F(X \times J))$ and $((\partial Y) \times J, F((\partial X) \times J))$ are locally unknotted.

3. For every subinterval $J$ of $I,(Y \times J, F(X \times J))$ and $(Y \times J, F(X \times J))$ are locally unknotted.

To obtain the codimension $\geqq 3$ isotopy theorem, we use Lickorish's device of converting concordance problems to cone problems by glueing cones on top. 
TheOREM 16. Let $F: X \times I \rightarrow Y \times I$ be an isotopy with $X$ compact and $\left(Y, F_{0}(X)\right)$ a codimension $\geqq 3$ pair. Then $F$ can be covered by an ambient isotopy iff

$$
F^{-1}\left(I^{i}(Y) \times I\right)=F_{0}^{-1}\left(I^{i}(Y)\right) \times I .
$$

Proof. Necessity is clear and for sufficiency, we prove that in such a situation, $(Y \times 0, F(X \times 0))<(Y \times I, F(X \times I))$ is locally collared and $d(F(x, t) ; Y \times I, F(X \times I))$ is constant for $0<t<1$.

By applying this result to $F^{s}$, defined by $F^{s}(x, t)=F(x, \min (t+s, 1))$ and $F^{-s}$, defined by $F^{-s}(x, t)=F(x, \max (s-t, 0))$ gives local collaring. Note that by hypotheses $F^{-1}\left(I^{i}(Y) \times I\right)=F_{0}^{-1}\left(I^{i}(Y)\right) \times I$ so in particular, $F_{0}^{-1}\left(I^{i}(Y)\right)=F_{s}^{-1}\left(I^{i}(Y)\right)$ and hence, $\left(Y, F_{s}(X)\right)$ is a codimension $\geqq 3$ pair and $F^{s}$ and $F^{-s}$ satisfy the assumptions of the theorem.

As usual, identify $X$ with $F_{0}(X)$ and $F$ with $F\left(F_{0} \times I\right)^{-1}$ and we will assume that $X<Y$ and $Y$ compact. Triangulate $(Y, X)$ and subdivide so that $F: \alpha K \times I \rightarrow \beta J \times I$ is simplicial with $\alpha K \times I$ cylindrical. Let $\alpha K \times 0=K$ and $\beta J \times 0=J$. Let $\alpha K \times 1=K_{1}$ and $\beta J \times 1=J_{1}$. Extend $F$ to

$$
\bar{F}: \alpha K \times I \cup c K_{1} \rightarrow \beta J \times I \cup c J_{1}
$$

by coning $F \mid K_{1}: K_{1} \rightarrow J_{1}$. We can construct homeomorphisms

$$
\begin{gathered}
\phi_{\alpha}: \alpha K \times I \cup c K_{1} \rightarrow c K \text { rel } K, \\
\phi_{B}: \beta J \times I \cup c J_{1} \rightarrow c J \operatorname{rel} J,
\end{gathered}
$$

and such that for $A \in K$ (resp. $A \in J)$

$$
\phi_{\alpha}(A \times I \cup c(A \times 1))=c A\left(\operatorname{resp} . \phi_{\beta}(A \times I \cup c(A \times 1))=c A\right) .
$$

Just order the simplices of $K$ (or $J$ ) in increasing order of dimension and construct $\phi_{\alpha}\left(\right.$ or $\left.\phi_{\beta}\right)$ inductively.

Let $G: c K \rightarrow c J=\phi_{\beta} \bar{F} \phi_{\alpha}^{-1}$.

We wish to apply Theorem V.4 to $G$. We already know that $(J, K)$ is a codimension $\geqq 3$ pair.

$$
\phi_{\beta}^{-1}\left(c I^{i}(J)\right)=I^{i}(J) \times I \cup c I^{i}\left(J_{1}\right)
$$

because $I^{i}(J)$ is a subcomplex of $J$. So

$$
\begin{aligned}
\bar{F}^{-1} \phi_{\beta}^{-1}\left(c I^{i}(J)\right) & =F^{-1}\left(I^{i}(J) \times I\right) \cup c F_{1}^{-1}\left(I^{i}\left(J_{1}\right)\right) \\
& =F_{0}^{-1}\left(I^{i}(J)\right) \times I \cup c F_{1}^{-1}\left(I^{i}\left(J_{1}\right)\right) .
\end{aligned}
$$

Since $F_{1}^{-1}\left(I^{i}\left(J_{1}\right)\right)=F_{0}^{-1}\left(I^{i}(J)\right)$, and $F_{0}^{-1}\left(I^{i}(J)\right)$ is a subcomplex of $K$, it follows that

$$
G^{-1}\left(c I^{i}(J)\right)=\phi_{\alpha} \bar{F}^{-1} \phi_{\beta}^{-1}\left(c I^{i}(J)\right)=c F_{0}^{-1}\left(I^{i}(J)\right) .
$$

Hence, Theorem V.4 applies to $G$.

We immediately obtain the local collaring result from Theorem V.4. And from Corollary IV.6 we have that $G$ is conewise homogenous. 
If $x \in K$, then $x \in$ Int $A$ for a unique $A \in K$. If $\overline{c x}$ is the cone line segment in $c K$, then Int $\phi_{\alpha}^{-1} \overline{c x} \subset$ Int $A \times I \cup c A$ by hypothesis on $\phi_{\alpha}$, and $\phi_{\alpha}^{-1} \overline{c x}$ joins $c$ to $x$. Since $K \times I$ is a cylindrical triangulation, every simplex of Int $\alpha A \times I$ separates Int $A \times I \cup c A$, with $x$ in the closure of the "bottom" component and $c$ in the closure of the "top" component. It follows that Int $\phi_{\alpha}^{-1} \overline{c x}$ meets the interior of every simplex of $\alpha A \times I-\partial \alpha A \times I$.

From this, and the fact that $G$ is $\mathrm{CH}$, we get that $d(F(y) ; J \times I, F(K \times I))=$ $d\left(F(y) ; J \times I \cup c J_{1}, \bar{F}\left(K \times I \cup c K_{1}\right)\right)$, for $y \in K \times I-K \times I$, is constant for $y \in \operatorname{Int} A \times I$. In particular,

$$
d(F(x, t) ; J \times I, F(K \times I))
$$

is constant for $0<t<1$.

This proves the case where $Y$ is compact. The extension to the noncompact case is identical to the extension at the end of the proof of Theorem 7.

As a corollary, we have the following unpublished result of Hudson:

Corollary 17. Let $F: X \times I \rightarrow Y \times I$ be an isotopy with $Y$ an n-manifold and $X$ compact such that

1. $F^{-1}(\partial Y \times I)=F_{0}^{-1}(\partial Y) \times I$,

2. $n-\operatorname{dim} X \geqq 3$,

3. $n$-dim $F_{0}^{-1}(\partial Y) \geqq 4$.

Then $F$ can be covered by an ambient isotopy.

VII. Extending triangulations. For a polyhedral pair $\left(X, X_{0}\right)$ if $k_{0}: K_{0} \rightarrow X_{0}$ is a triangulation of $X_{0}$, then we will say that $k: K \rightarrow X$ extends $\left(K_{0} ; k_{0}\right)$ if the induced map

$$
K_{0} \stackrel{k_{0}}{\longrightarrow} X_{0} \subset X \stackrel{k}{\longleftarrow} K
$$

is simplicial. In this section we will generalize Armstrong's results [A2] about extending triangulations from manifold pairs to polyhedral pairs.

LEMMA 1. For a compact polyhedral pair $\left(X, X_{0}\right)$ any triangulation $\left(K_{0} ; k_{0}\right)$ has an iterated derived subdivision which extends to a triangulation of $X$.

Proof. If $(K ; k)$ is any triangulation of $X$, then we can subdivide so that $\alpha K_{0} \rightarrow X_{0} \subset X \leftarrow \beta K$ is simplicial. There is a further subdivision $\gamma \alpha K_{0}$ which is an iterated derived $\eta^{(r)} K_{0}$, [Z; Lemma 4] or [H; Corollary 1.6]. We can extend $\gamma$ over $\beta K$ to obtain a subdivision $\delta \beta K$ so that $(\delta \beta K ; k)$ extends $\left(\eta^{(r)} K_{0} ; k_{0}\right)$.

THEOREM 2. Let $\left(X, X_{0}\right)$ be a compact polyhedral pair and let $\left(K_{0} ; k_{0}\right)$ be a triangulation of $X_{0} .\left(K_{0} ; k_{0}\right)$ extends to a triangulation of $X$ iff $k_{0}^{-1} I^{i}\left(X, X_{0}\right)$ is a subcomplex of $K_{0}$ for all $i$.

Proof. Necessity is clear.

By Lemma 1, there exists some iterated derived subdivision of $K_{0}$ which will extend. Since a derived subdivision is obtained by a number of stellar subdivisions, 
to prove sufficiency we use induction on the number of stellar subdivisions and reduce the proof to that of the theorem with the additional assumption that $\left(\sigma K_{0} ; k_{0}\right)$ extends to a triangulation of $X$, where $\sigma K_{0}$ is the subdivision obtained by starring $K_{0}$ at $A$.

Let $k: K \rightarrow X$ be a triangulation extending $k_{0}: \sigma K_{0} \rightarrow X_{0}$. Let $s: \sigma K_{0} \rightarrow K$ be the simplicial embedding, $k^{-1} k_{0}$. We assume that $s \sigma K_{0}$ is full in $K$, by deriving $\bmod s \sigma K_{0}$ if necessary.

Let $u=s(\sigma A)$ where $\sigma A$ is the vertex in Int $A$ introduced by $\sigma$, and let $v$ be a vertex of $s A$. Note that the hypothesis on the triangulation $K_{0}$ implies that $d\left(x ; K, s K_{0}\right)$ is constant for $x$ in the interior of $s A$ so that $u$ is a nice face of $u v$ in $\left(K, s \sigma K_{0}\right)$.

By deriving mod $s \sigma K_{0}$ again if necessary, we can assume that $v \mathrm{Lk}(u v ; K)$ is a regular neighborhood of $v \mathrm{Lk}\left(u v ; s \sigma K_{0}\right) \bmod \mathrm{Lk}\left(u v ; s \sigma K_{0}\right)$ in $\mathrm{Lk}(u ; K)$. Under this assumption, clearly, $v \mathrm{Lk}\left(u v ; K, s \sigma K_{0}\right)$ is a regular neighborhood of

$$
v \operatorname{Lk}\left(u v ; s \sigma K_{0}\right) \bmod \mathrm{Lk}\left(u v ; s \sigma K_{0}\right)
$$

in $\operatorname{Lk}\left(u ; K, s \sigma K_{0}\right)$. If $C \in \mathrm{Lk}\left(u v ; s \sigma K_{0}\right)$ then $u C$ is a nice face of $u v C$ in $\left(K, s \sigma K_{0}\right)$ (since both $u C$ and $u v C$ are images of simplices interior to a simplex of $K_{0}$ ). Hence, by Corollary II.17, $C$ is a nice face of $v C$ in $\mathrm{Lk}\left(u ; K, s \sigma K_{0}\right)$. Hence, the collapse $\left(v \operatorname{Lk}\left(u v ; s \sigma K_{0}\right) \mid \operatorname{Lk}\left(u v ; s \sigma K_{0}\right)\right) \searrow v$ conewise is homogenous in $\operatorname{Lk}\left(u, K, s \sigma K_{0}\right)$. It follows from Theorem III.6 that $v$ Lk $\left(u v ; K, s \sigma K_{0}\right)$ is a regular neighborhood of $v$ in $\mathrm{Lk}\left(u ; K, s \sigma K_{0}\right)$.

By Lemma II.14a, there exists a homeomorphism

$$
\phi: \operatorname{Lk}\left(u ; K, s \sigma K_{0}\right) \cong(v+x) \operatorname{Lk}\left(u v ; K, s \sigma K_{0}\right) .
$$

Since $v \mathrm{Lk}\left(u v ; K, s \sigma K_{0}\right)$ is a regular neighborhood of $v$ in $\mathrm{Lk}\left(u ; K, s \sigma K_{0}\right)$, we can assume that $\phi$ is the identity on $v \operatorname{Lk}(u v ; K)$, using the method of proof of Lemma II.14b.

If $P$ is the pair $\mathrm{Lk}\left(u ; K, s \sigma K_{0}\right)-\mathrm{St}^{\circ}(v ; K)$, then

$$
\phi(P)=x \operatorname{Lk}\left(u v ; K, s \sigma K_{0}\right) \operatorname{rel~Lk}\left(u v ; K, s \sigma K_{0}\right) .
$$

By coning, this extends to a homeomorphism

$$
\mathrm{St}\left(u ; K, s \sigma K_{0}\right) \cong u(v+x) \operatorname{Lk}\left(u v ; K, s \sigma K_{0}\right)
$$

rel $u v \operatorname{Lk}(u v ; K)=\operatorname{St}(u v ; K)$. Now homeomorph $u(v+x) \cong v x$ rel $(v+x)$ and join, to get a homeomorphism

$$
\phi: \mathrm{St}\left(u ; K, s \sigma K_{0}\right) \cong v x \mathrm{Lk}\left(u v ; K, s \sigma K_{0}\right)
$$

rel $v \operatorname{Lk}(u v ; K)$. Note that $\phi(P)=\phi(P)=x \mathrm{Lk}\left(u v ; K, s \sigma K_{0}\right)$.

What $\phi$ really is, is best illustrated by looking at $K_{0}$.

$$
\text { St }\left(A ; K_{0}\right)=A \operatorname{Lk}\left(A ; K_{0}\right)=v_{0} B \operatorname{Lk}(A ; K)
$$


and

$$
\sigma \mathrm{St}\left(A ; K_{0}\right)=u_{0} \dot{A} \operatorname{Lk}\left(A ; K_{0}\right)=u_{0}\left(v_{0} \dot{B}+B\right) \operatorname{Lk}(A ; K) \cong u_{0}\left(v_{0}+x\right) \dot{B} \operatorname{Lk}\left(A ; K_{0}\right)
$$

(where $s u_{0}=u, s v_{0}=v$ ). Thus,

$$
\operatorname{Lk}\left(u ; s \sigma K_{0}\right)-\mathrm{St}^{\circ}\left(v ; s \sigma K_{0}\right)=s(B \operatorname{Lk}(A ; K)) .
$$

At this point, we are motivated, having read Armstrong's paper, to introduce the complex

$$
K_{1}=\left(K-\mathrm{St}^{\circ}(u ; K)\right) \cup v *\left(\operatorname{Lk}(u ; K)-\mathrm{St}^{\circ}(v ; K)\right),
$$

where the intersection of the two terms is

$$
\operatorname{Lk}(u ; K)=\left(\operatorname{Lk}(u ; K)-\mathrm{St}^{\circ}(v ; K)\right) \cup v * \operatorname{Lk}(u v ; K) .
$$

We will define a homeomorphism $g: K \rightarrow K_{1}$ such that $\left(K_{1} ; \mathrm{kg}^{-1}\right)$ is a triangulation of $X$ extending $\left(K_{0} ; k_{0}\right)$.

Begin by letting $g \mid K-\mathrm{St}^{\circ}(u ; K)=$ identity. So we must define $g$ over St $(u ; K)$.

First, on $\mathrm{St}\left(u ; s \sigma K_{0}\right)=s\left(v_{0} B \operatorname{Lk}\left(A ; K_{0}\right)\right)$ define $g$ to be the composite:

$$
\mathrm{St}\left(u ; s \sigma K_{0}\right) \stackrel{s^{-1}}{\longrightarrow} v_{0} B \operatorname{Lk}\left(A ; K_{0}\right) \stackrel{v * s}{\longrightarrow} v\left(\operatorname{Lk}\left(u ; s \sigma K_{0}\right)-\operatorname{St}\left(v ; s \sigma K_{0}\right)\right) \text {. }
$$

Note that this extends the identity on $\operatorname{Lk}\left(u ; s \sigma K_{0}\right)=\left(\operatorname{Lk}\left(u ; s \sigma K_{0}\right)-\operatorname{St}^{\circ}\left(v ; s \sigma K_{0}\right)\right)$ $\cup v^{*} \mathrm{Lk}\left(u v ; s \sigma K_{0}\right)$, on the latter term because $s$ is simplicial and hence $=v * s$.

Let $j: v x \operatorname{Lk}\left(u v ; s \sigma K_{0}\right) \rightarrow v x \operatorname{Lk}\left(u v ; s \sigma K_{0}\right)$ be the embedding $(v * \phi) g(\phi)^{-1}$. This is a homeomorphism $\operatorname{rel} v \mathrm{Lk}\left(u v ; s \sigma K_{0}\right) \cup x \mathrm{Lk}\left(u v ; s \sigma K_{0}\right)$.

Assuming for a moment that $j$ is conewise homogenous, regarded as an embedding into $v x \mathrm{Lk}(u v ; K)$, then $j$ can be covered by a homeomorphism

$$
J: v x \operatorname{Lk}(u v ; K) \rightarrow v x \operatorname{Lk}(u v ; K) \operatorname{rel}(v+x) \operatorname{Lk}(u v ; K)
$$

by Corollary IV. 8 and the remarks at the end of $\S I V$.

So letting $g=(v * \phi)^{-1} J \phi$ on St $(u ; K)$ extends the previous definitions of $g$ and $\left(K ; \mathrm{kg}^{-1}\right)$ extends $\left(K_{0} ; k_{0}\right)$ because $\left(\mathrm{kg}^{-1}\right)^{-1} k_{0}=g s$ is simplicial on $K_{0}$.

Thus, it suffices to show that regarded as an embedding $j: v x \operatorname{Lk}\left(u v ; s \sigma K_{0}\right)$ $\rightarrow v x \mathrm{Lk}(u v ; s \sigma K)$ is conewise homogenous (with respect to either cone structure). It suffices to show that $j^{-1}$, regarded as an embedding is $\mathrm{CH} . j^{-1}=\phi g^{-1}(v * \phi)^{-1}$. If we use the $v$-cone structure then since $\phi$ is a homeomorphism and $(v * \phi)^{-1}$ $=v *\left(\phi^{-1}\right)$ is a cone map, it suffices to show that

$$
g^{-1}: v\left(\operatorname{Lk}\left(u ; s \sigma K_{0}\right)-\mathrm{St}^{\circ}\left(v ; s \sigma K_{0}\right)\right) \rightarrow \mathrm{St}(u ; K)
$$

is $\mathrm{CH}$ and since $(v * s)^{-1}=v * s^{-1}$ is a cone map, that

$$
s: v_{0} B \operatorname{Lk}\left(A ; K_{0}\right) \rightarrow \operatorname{St}(u ; K)
$$

is $\mathrm{CH}$. This is clear because every open cone-line of $v_{0} B \mathrm{Lk}\left(A ; K_{0}\right)$ lies in the interior of a simplex of $K_{0}$ and by hypothesis $d\left(x ; K, s K_{0}\right)$ is constant on Int $s C$ for $C \in K_{0}$. 
Thus, $j$ is $\mathrm{CH}$ and the theorem is proved.

COROLlaRY 3. For a compact polyhedral pair $\left(X, X_{0}\right)$ the following are equivalent:

a. $\left(X, X_{0}\right)$ is locally unknotted.

b. Every ambient isotopy of $X_{0}$ extends to an ambient isotopy of $X$.

c. Every triangulation of $X_{0}$ extends to a triangulation of $X$.

Proof. $\mathrm{a} \rightarrow \mathrm{b}$. Corollary VI.

$\mathrm{a} \rightarrow \mathrm{c}$. $I^{i}\left(X, X_{0}\right)=I^{i}\left(X_{0}\right)$ which is a subcomplex of any triangulation of $X_{0}$.

$\mathrm{b} \rightarrow \mathrm{a} . \mathrm{b}$ gives immediately the fact that $x \sim y$ in $X_{0}$ implies $x \sim y$ in $\left(X, X_{0}\right)$. Apply Theorem II.23.

$\mathrm{c} \rightarrow \mathrm{a}$. If $d\left(x ; X_{0}\right)=i$ then $X_{0}$ can be triangulated with $x$ in the interior of an $i$-simplex. By c the same is true of $\left(X, X_{0}\right)$ and hence $d(x ; X)=d\left(x ; X, X_{0}\right)$.

Actually, the following more general corollary is true. We leave the verification to the reader:

COROllary 3'. Let $Y<X_{0}<X$ be compact polyhedra. The following are equivalent:

a. $\left(X, X_{0}\right)$ is locally unknotted at every point of $X_{0}-Y$.

b. Every ambient isotopy of $X_{0}$ rel $Y$, extends to an ambient isotopy of $X$.

c. If $\left(K_{0}, L ; h\right)$ is a triangulation of $\left(X_{0}, Y\right)$ then if $(L ; h \mid L)$ extends to a triangulation of $\left(X, X_{0}\right)$, then $\left(K_{0} ; h\right)$ extends to a triangulation of $X$.

Theorem 2 and Corollary 3 answer some questions raised at the end of [A2] and have been stated in some form by Zeeman.

\section{Homogenous homotopy and general position theorems.}

Definition 1. Call a homotopy $F: X \times I \rightarrow Y$ homogenous if for every $x \in X$, $d(F(x, t) ; Y)$ is constant for $0 \leqq t \leqq 1$.

Thus, a homogenous homotopy of a map is a homotopy which preserves intrinsic dimension in the image. Equivalently, $F: X \times I \rightarrow Y$ is homogenous if for each $i$, $F^{-1}\left(I^{i}(Y)\right)=F_{0}^{-1}\left(I^{i}(Y)\right) \times I$.

We will consider in this section theorems about "improving" a map through homogenous homotopy, i.e. minimizing self-intersection dimensions and making maps nondegenerate.

THEOREM 2. Let $f: X \rightarrow Y$ be a p.l. map with $X$ compact and $F: X_{0} \times I \rightarrow Y$ a homogenous homotopy of $f \mid X_{0}$, where $X_{0}<X$. Then there exists a homogenous homotopy $G: X \times I \rightarrow Y$ with $G_{0}=f$ and $G \mid X_{0} \times I=F$.

Proof. Let $i: X \rightarrow B^{n}$ be an embedding of $X$ into Int $B^{n}$ with $n$-dim $X \geqq 3$, then $F \times i: X_{0} \times I \rightarrow Y \times B_{n} \times I$ defined by $F \times i(x, t)=(F(x, t), i(x), t)$ is a homogenous isotopy with $\left(Y \times B^{n}, F_{0} \times i\left(X_{0}\right)\right)$ a codimension $\geqq 3$ pair.

Hence, by Theorem VI.16, it extends to an ambient isotopy $H: Y \times B^{n} \times I$ $\rightarrow Y \times B^{n} \times I$. Let $G(x, t)=\operatorname{proj}_{Y} H(f(x), i(x), t)$. 
Note that the usual proof of the homotopy extension theorem, by finding a retraction of $X \times I$ to $X_{0} \times I \cup X \times 0$ fails because the resulting homotopy fails to be homogenous.

Our first application is to obtain a nondegeneracy theorem, using Theorem 2 to go by induction up the intrinsic skeleta. The inductive step requires a sharpening of the usual nondegeneracy theorem.

Lemma 3. Let $\left(X, X_{0}\right)$ and $(M, Y)$ be compact polyhedral pairs and $f: X \rightarrow M$ a p.l. map with $f^{-1}(Y) \subset X_{0},(M, Y)$ a relative n-manifold, $f \mid X_{0}$ nondegenerate, $\operatorname{dim} X \leqq n$. Then there exists a homotopy $F$ of $f$ rel $X_{0}$ with $F\left(\left(X-X_{0}\right) \times I\right) \subset M-Y$ and $F_{1}$ nondegenerate.

Proof. Triangulate to obtain: $f: K \rightarrow M^{n}$, simplicial, with $K_{0}$ a full subcomplex of $K, f \mid K_{0}$ nondegenerate. $L$ a full subcomplex of $M$ with $f^{-1} L<K_{0}$, and $(M, L)$ a relative $n$-manifold.

Order the simplices of $M-L$ in decreasing order of dimension $A_{1}, \ldots, A_{p}$. Let $\eta K$ and $\eta M$ be deriveds such that $f$ is simplicial. Define $D(A ; f)=f^{-1} D(A ; M)$ and $\dot{D}(A ; f)=f^{-1} \dot{D}(A ; M)$ following Cohen.

$D(A ; f)$ is a subcomplex of $f^{-1} \eta A * \dot{D}(A ; f)$, with $f \mid D(A ; f)$ the restriction of the join of $f^{-1} \eta A \rightarrow \eta A$ with $f: \dot{D}(A ; f) \rightarrow \dot{D}(A ; M)$.

We proceed by a double induction.

Define $F(0): K \times I \rightarrow M$ to be $f\left(\operatorname{proj}_{K}\right)$.

Inductively, we define $F(i): K \times I \rightarrow M$ to satisfy:

1. $F(i)_{0}=F(i-1)_{1}$ and $F(i) \mid\left(\bigcup_{j<i} D\left(A_{j} ; f\right) \cup K_{0}\right) \times I$ is the restriction of

$$
F(i-1)_{1}\left(\operatorname{proj}_{K}\right) \text {. }
$$

2. For all $j, F(i)\left(\left(D\left(A_{j} ; f\right)-\dot{D}\left(A_{j} ; f\right)\right) \times I\right) \subset D\left(A_{j} ; M\right)-\dot{D}\left(A_{j} ; M\right)$.

3. $F(i)_{1} \mid D\left(A_{i} ; f\right)$ is nondegenerate.

4. For all $j>i, F(i)_{1}$ is the map $D\left(A_{j} ; f\right) \rightarrow D\left(A_{j} ; M\right)$ defined by the restriction of the join of $f^{-1} \eta A_{j} \rightarrow \eta A_{j}$ and $F(i)_{1} \mid \dot{D}\left(A_{j} ; f\right): \dot{D}\left(A_{j} ; f\right) \rightarrow \dot{D}\left(A_{j} ; M\right)$.

Assuming $F(1), \ldots, F(p)$ are defined then the composite homotopy $F(1)+\cdots$ $+F(p)$ is a homotopy of $f\left(\right.$ since $\left.(F(1)+\cdots+F(p))_{0}=F(1)_{0}=f\right)$ to a nondegenerate map (namely $F(p)_{1}$, by 1 and 3 ) which is rel $K_{0}$ (by 1$)$ and maps $\left(K-K_{0}\right) \times I$ into $M-L$ (by 2).

Now assuming $F(i-1)$ is constructed, define $F(i)$ on $\left(\cup_{j<i} D\left(A_{i} ; f\right) \cup K_{0}\right) \times I$ $\cup K \times 0$ by condition 1 . Note that $F(i-1)_{1}$ is nondegenerate on $\bigcup_{j<i} D\left(A_{i} ; f\right) \cup K_{0}$.

To define $F(i)$ on $D\left(A_{i} ; f\right) \times I$ note that $F(i-1)_{1}$ on $D\left(A_{i} ; f\right)$ is the restriction of the join of $f^{-1} \eta A_{i} \rightarrow \eta A_{i}$ with the nondegenerate map $F(i-1)_{1}: \dot{D}\left(A_{i} ; f\right) \rightarrow$ $\dot{D}\left(A_{i} ; M\right) . D\left(A_{i} ; M\right)$ is a ball of dimension $n-\operatorname{dim} A_{i}$ which is $\geqq \operatorname{dim} D\left(A_{i} ; f\right)$ $=\bigcup\left\{D(B ; K): f B=A_{i}\right\}$, since $\operatorname{dim} K \leqq \operatorname{dim} M$, and $\operatorname{dim} B \geqq \operatorname{dim} A_{i}$ for all such $B$. Hence, by standard general position on Euclidean space arguments, we can find a homotopy $F(i): D\left(A_{i} ; f\right) \times I \rightarrow D\left(A_{i} ; M\right)$ rel $\dot{D}\left(A_{i} ; f\right)$, mapping $\left(D\left(A_{i} ; f\right)\right.$ $\left.D\left(A_{i} ; f\right)\right) \times I$ into $D\left(A_{i} ; M\right)-\dot{D}\left(A_{i} ; M\right)$ and such that $F(i)_{1}$ is nondegenerate on $D\left(A_{i} ; f\right)$. 
To define $F(i)$ on $D\left(A_{j} ; f\right) \times I$ for $j>i$ proceed by induction up the sequence $A_{i+1}, \ldots, A_{p}$. Inductive assumption and condition 4 determine

$$
F(i) \times I: \dot{D}\left(A_{j} ; f\right) \times I \cup D\left(A_{j} ; f\right) \times \dot{I} \rightarrow \dot{D}\left(A_{j} ; M\right) \times I \cup D\left(A_{j} ; M\right) \times I .
$$

By following this by a homeomorphism to $\dot{\Delta}$, with $\operatorname{dim} \Delta=n-\operatorname{dim} A_{j}+1$, and subdividing to make the map simplicial, we can use linearity and the fact that $D\left(A_{j} ; f\right)$ is a subcomplex of $f^{-1} A_{j} * \dot{D}\left(A_{j} ; f\right)$ and $F(i)_{0}$ and $F(i)_{1}$ are "cone maps" to extend this to a map taking the rest of $D\left(A_{j} ; f\right) \times I$ to Int $D\left(A_{j} ; M\right) \times I$. Projecting to $D\left(A_{j} ; M\right)$ extends $F(i)$ over $D\left(A_{j} ; f\right) \times I$ to satisfy 2 and 4 .

THEOREM 4. Let $f: X \rightarrow Y$ be a map, nondegenerate on $X_{0}<X$, with $\mathrm{Cl}\left(X-X_{0}\right)$ compact and $\operatorname{dim} f^{-1}\left(I^{i}(Y)\right) \leqq i$, then $f$ can be homogenously homotoped to a nondegenerate map, rel $X_{0}$.

Proof. By replacing $X$ by $(X)_{R}, X_{0}$ by $\left(X_{0}\right)_{R}$ and $Y$ by a regular neighborhood of $f\left((X)_{R}\right)$, if necessary, we can assume $X, X_{0}$ and $Y$ are compact. With this assumption we proceed by induction up the intrinsic skeleta of $Y$. More precisely, let $m_{f}=\min \left\{i: X-X_{0} \subset f^{-1}\left(I^{i}(Y)\right)\right\}$. Assuming the result for maps $f$, with $m_{f}<n$, we prove it for $m_{f}=n$.

By inductive hypothesis, we can homogenously homotope $f \mid f^{-1} I^{n-1}(Y)$ to a nondegenerate map, rel $X_{0}$. By Theorem 2, this extends to a homogenous homotopy rel $X_{0}$ of $f$ to a map $\bar{f}$ nondegenerate on $X_{0} \cup f^{-1}\left(I^{n-1}(Y)\right) .\left(X, X_{0} \cup f^{-1} I^{n-1}(Y)\right)$ and $\left(I^{n}(Y), I^{n-1}(Y)\right)$ satisfy the hypotheses of Lemma 3, with $\bar{f}: X \rightarrow I^{n}(Y)$, since $m_{f}=n$ and the homotopy of $f$ to $\bar{f}$ was homogenous. Hence, by Lemma 3, we can homotope rel $X_{0} \cup f^{-1} I^{n-1}(Y)$ with $X-X_{0} \cup f^{-1} I^{n-1}(Y)$ kept in $I^{n}(Y)-I^{n-1}(Y)$ and go from $\bar{f}$ to a nondegenerate map, $g$. The composite homotopy $f \sim \bar{f} \sim g$ is the required homotopy of $f$ to a nondegenerate map.

We will say that two subspaces $X$ and $Y$ are in general position in a polyhedron $Q$, if $\operatorname{dim} X \cap Y-I^{i-1}(Q) \leqq \operatorname{dim} X+\operatorname{dim} Y-i$, for all $i$.

To prove that we can ambient isotope subspaces to general position, we need the analogue of Lemma 3, for general position.

Lemma 5. Let $(M, Y)$ be a compact, relative $n$-manifold with $X_{0}, X, R_{1}, \ldots$, $R_{q}<M$ and $Y \cap X<X_{0}$. Then there is an ambient isotopy of identity on $M$ to $f: M \rightarrow M$ rel $X_{0} \cup Y$ such that $\operatorname{dim} f\left(X-X_{0}\right) \cap R_{i} \leqq \operatorname{dim} X-X_{0}+\operatorname{dim} R_{i}-n$.

Proof. Triangulate $\left(X, X_{0}\right)$ and order the simplices of $X-X_{0}$ in increasing order of dimension $A_{1}, \ldots, A_{p}$. Proceed inductively with the $i$ th inductive step an ambient isotopy of $M \operatorname{rel} X_{0} \cup Y \cup A_{1} \cup \cdots \cup A_{i-1}$ moving the image of $A_{i}$ under the $i-1$ st step mod its boundary to something in general position with respect to the $R_{j}$ 's. This is done by restricting the motion to be supported by a regular neighborhood of $f_{i-1}\left(A_{i}\right) \bmod f_{i-1}\left(\dot{A}_{i}\right)$. This will define $f_{i}$ and the composite ambient isotopy to $f_{p}$ will be the required isotopy. 
TheOREM 6. Let $X, X_{0}, R_{1}, \ldots, R_{q}<Q$ with $\mathrm{Cl} X-X_{0}$ compact. Then there exists an ambient isotopy rel $X_{0}$ of identity on $Q$ to $f: Q \rightarrow Q$ with $f\left(X-X_{0}\right)$ in general position with respect to each of the $R_{i}$ 's.

Proof. By replacing $X$ by $(X)_{R}, Q$ by a regular neighborhood $V$ of $(X)_{R}$ in $Q$, $X_{0}$ by $\dot{V} \cup\left(X_{0} \cap V\right)$ and $R_{i}$ by $R_{i} \cap V$, if necessary, we can assume that $Q$ is compact. With this assumption we proceed by induction on

$$
m_{X}=\min \left\{i: X-X_{0} \subset I^{i}(Q)\right\} .
$$

Assuming the result when $m_{X}<n$, we prove it for $m_{X}=n$.

By inductive hypothesis there is an ambient isotopy rel $X_{0}$ of $Q$ to $f_{1}: Q \rightarrow Q$ such that $f_{1}\left(X \cap I^{n-1}(Q)-X_{0}\right)$ is in general position with respect to the $R_{i}$ 's.

By Lemma 5, we can ambient isotope $I^{n}(Q)$ rel $\left(X_{0} \cap I^{n}(Q)\right) \cup I^{n-1}(Q)$ to $g: I^{n}(Q) \rightarrow I^{n}(Q)$ so that $g f_{1}\left[X-\left(\left(X_{0} \cap I^{n}(Q)\right) \cup I^{n-1}(Q)\right)\right]$ is in general position with respect to the $R_{i} \cap I^{n}(Q)$.

By Corollaries VI.11 and VI.12, this extends to an ambient isotopy of $Q$. So the composite of the isotopy to $f_{1}$ and this extension give the required ambient isotopy.

Hudson's proof of [H; Lemma 4.7] produces general position for maps, using general position for subspaces. The homotopies he constructs are ambient isotopies on their supports. In a polyhedron, consider a homotopy $F:\left(X_{0} \cup X_{1}\right) \times I \rightarrow Y$, which is constant on $X_{0} \times I$ and which on $X_{1} \times I$ is equal to $G \cdot\left(F_{0} \times I\right)$ where $G: Y \times I \rightarrow Y$ is an ambient isotopy. Such a homotopy is clearly homogenous. Thus, Hudson's method of proof using Theorem 6, instead of [H; Lemma 4.6] gives the following analogue of [H; Lemma 4.7]:

THEOREM 7. Let $\left(K, K_{0}\right)$ be a complex pair with $K-K_{0}$ finite and $f: K \rightarrow Q$ and a map which embeds each simplex of $K$ in the polyhedron $Q$, and $R_{1}, \ldots, R_{q}<Q$. Then there is a homogenous homotopy rel $K_{0}$ of $f$ to $f^{\prime}: K \rightarrow Q$ such that:

1. $f^{\prime}$ embeds each simplex of $K$.

2. If $A_{1}, \ldots, A_{r}$ are distinct simplices of $K$, then

$$
\begin{gathered}
\operatorname{dim} \bigcap_{i=1}^{r} f^{\prime}\left(\operatorname{Int} A_{i}\right)-I^{m-1}(Q) \leqq \sum_{i=1}^{r} \operatorname{dim} A_{i}-(r-1) m, \\
\operatorname{dim} \bigcap_{i=1}^{r} f^{\prime}\left(\operatorname{Int} A_{i}\right) \cap R_{j}-I^{m-1}(Q) \leqq \sum_{i=1}^{r} \operatorname{dim} A_{i}+\operatorname{dim} R_{j}-r m,
\end{gathered}
$$

for all $j$ and $m$.

In particular, if $f: X \rightarrow Q$ is a nondegenerate map with $\operatorname{dim} S_{r}(f)-f^{-1} I^{m-1}(Q)$ $\leqq r \operatorname{dim} X-(r-1) m$ for all $r$ and $m$, then we call $f$ a general position map.

CoROllary 8. Let $f: X \rightarrow Q$ be a map with $f \mid X_{0}$ a general position map $X_{0}<X$ (compact), then $f$ can be homogenously homotoped rel $X_{0}$ to a general position map.

Examining this outline of a theory of general position for polyhedra, we see two major flaws. 
First, we do not have simplicial or p.l. approximation theorem, i.e. let $f: X \rightarrow Q$ be a topological map of polyhedra such that for all $m, f^{-1} I^{m}(Q)$ is a subpolyhedron of $X$. Can we homogenously homotope $f$ to a p.l. map?

Secondly, our theory has no $\varepsilon$-approximation theorems. Lemmas 3 and 5 can probably be sharpened to get arbitrarily close approximations. Less obviously, Theorem 2 can be strengthened to extend small homotopies to small homotopies. Here the trick is to construct a retraction $r: X \times I \rightarrow X_{0} \times I \cup X \times 0$ such that $F \cdot r$ is a small homotopy and then use instead of $G, G \cdot r^{\prime}$, where $r^{\prime}: X \times I \rightarrow X \times I$ rel $X_{0}$ $\times I \cup X \times 0$ is a close approximation to $r$ and has the additional property that $G r^{\prime}(x, t) \sim G(x, t)$ in $Y$. This will introduce $\varepsilon$ 's into Theorem 4 and allow arbitrarily close approximation by nondegenerate maps. To introduce $\varepsilon$ 's into Theorem 6 and hence Theorem 7 one needs to have them in Theorem VI.11.

Stallings' treatment of general position in [S; Chapter V] is rather different from ours. His results on homotoping maps to general position are stronger than ours (and also more useful) but he has no ambient isotopy theorems and our results are the best possible there. To "localize" Stallings' argument and show its connection with our own we introduce:

Definition 8. Let $x \in X$, then define Stallings' dimension

$$
d_{S}(x ; X)=\max \{n: \operatorname{Lk}(x ; X) \text { is an }(n-2) \text {-connected } N D(n-1) \text { space }\} .
$$

From the properties of $N D(n)$ spaces developed in [S; Chapter V], it is clear that $d_{S}(x ; X) \geqq d(x ; X)$. Furthermore, $X$ is an $N D(n)$ space iff for all $x \in X$,

$$
d_{S}(x ; X) \geqq n \text {. }
$$

Letting $S^{i}(X)=\left\{x: d_{S}(x ; X) \leqq i\right\}$, we note that $S^{i}(X)<I^{i}(X)$ (it is a subpolyhedron because $x \sim y$ in $X$ implies $d_{S}(x ; X)=d_{S}(y ; X)$ and if $d_{S}(x ; X)=n$ then every point of $\mathrm{St}^{\circ}(x ; X)$ has as link an $(n-2)$ connected $N D(n-1)$ space and so has Stallings dimension at least $n$ ).

We would conjecture that a map $f: X \rightarrow Q$ can be homotoped to a map such that

$$
\operatorname{dim} S_{r}(f)-f^{-1} S^{m-1}(Q) \leqq r \operatorname{dim} X-(r-1) m .
$$

A proof along the lines of our proofs in this section would require the analogue of Theorem 2, for homotopies homogenous with respect to $d_{S}$, and relative versions of Stallings' theorems on the order of Lemma 3.

\section{BIBLIOGRAPHY}

[Z] E. C. Zeeman, Seminar on combinatorial topology, Notes Inst. Hautes Études Sci. Publ. Math. and The University of Warwick, Coventry, 1963.

[H] J. F. P. Hudson, Piecewise linear topology, Benjamin, New York, 1969.

[S] John R. Stallings, Lectures on polyhedral topology, Lecture Notes on Mathematics, Tata Institute of Fundamental Research, Bombay, 1968. 
[C] Marshall M. Cohen, A general theory of relative regular neighborhoods, Trans. Amer. Math. Soc. 136 (1969), 189-329.

[A1] M. A. Armstrong, Transversality for polyhedra, Ann. of Math. 86 (1967), 172-191.

[A2] - Extending triangulations, Proc. Amer. Math. Soc. 18 (1967), 701-704.

[L] W. B. R. Lickorish, The piecewise linear unknotting of cones, Topology 4 (1965), 67-91. [H-Z] J. F. P. Hudson and E. C. Zeeman, On combinatorial isotopy, Inst. Hautes Études Sci. Publ. Math. 19 (1964), 69-94.

[R] C. P. Rourke, Covering the track of an isotopy, Proc. Amer. Math. Soc. 18 (1967), 320-324.

[M] H. R. Morton, Joins of polyhedra, preprint, The University of Warwick, Coventry.

Princeton University,

Princeton, New Jersey 